Research review paper

\title{
Impact and application of electron shuttles on the redox (bio)transformation of contaminants: A review
}

\author{
Frank P. Van der Zee ${ }^{\mathrm{a}}$, Francisco J. Cervantes ${ }^{\mathrm{b}, *}$ \\ a IBB - Institute for Biotechnology and Bioengineering, Centre of Biological Engineering, University of Minho, Campus de Gualtar, 4710-057 Braga, Portugal \\ b División de Ciencias Ambientales, Instituto Potosino de Investigación Científica y Tecnológica (IPICYT), San Luis Potosí, SLP, Mexico
}

\section{A R T I C L E I N F O}

\section{Article history:}

Received 10 October 2008

Received in revised form 6 January 2009

Accepted 9 January 2009

Available online 23 January 2009

\section{Keywords:}

Degradation

Electron shuttle

Pollutants

Remediation

Redox mediator

Wastewater treatment

\begin{abstract}
A B S T R A C T
During the last two decades, extensive research has explored the catalytic effects of different organic molecules with redox mediating properties on the anaerobic (bio)transformation of a wide variety of organic and inorganic compounds. The accumulated evidence points at a major role of electron shuttles in the redox conversion of several distinct contaminants, both by chemical and biological mechanisms. Many microorganisms are capable of reducing redox mediators linked to the anaerobic oxidation of organic and inorganic substrates. Electron shuttles can also be chemically reduced by electron donors commonly found in anaerobic environments (e.g. sulfide and ferrous iron). Reduced electron shuttles can transfer electrons to several distinct electron-withdrawing compounds, such as azo dyes, polyhalogenated compounds, nitroaromatics and oxidized metalloids, among others. Moreover, reduced molecules with redox properties can support the microbial reduction of electron acceptors, such as nitrate, arsenate and perchlorate. The aim of this review paper is to summarize the results of reductive (bio)transformation processes catalyzed by electron shuttles and to indicate which aspects should be further investigated to enhance the applicability of redox mediators on the (bio)transformation of contaminants.
\end{abstract}

(c) 2009 Elsevier Inc. All rights reserved.

\section{Contents}

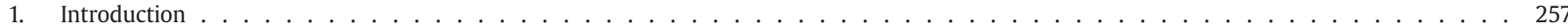

2. Role of redox mediators on azo dye reduction . . . . . . . . . . . . . . . . . . . . . . . . . . . . . . . 259

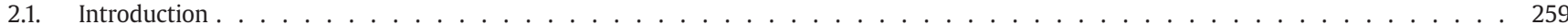

2.2. Redox mediator properties . . . . . . . . . . . . . . . . . . . . . . . . . . . . 259

2.2.1. Oxidation-reduction potential . . . . . . . . . . . . . . . . . . . . . . . . . . . 259

2.2.2. Ability to cross the cell membrane . . . . . . . . . . . . . . . . . . . . . . . . . . . . . . 263

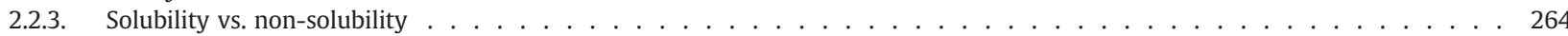

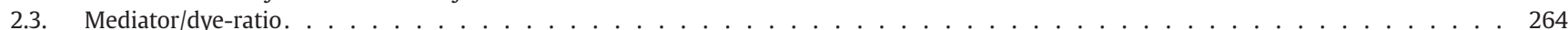

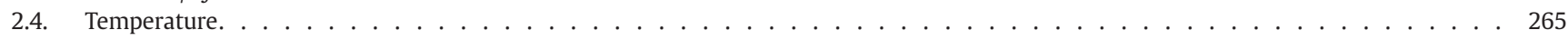

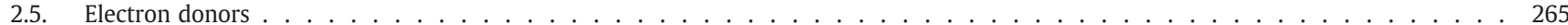

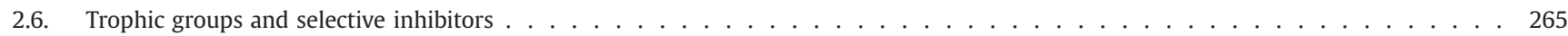

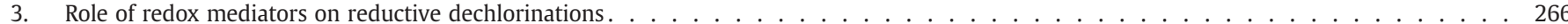

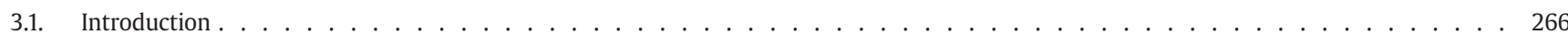

3.2. Main factors determining the input of RM in reductive dechlorinations. . . . . . . . . . . . . . . . . . . . . . . 266

3.2.1. $\mathrm{pH} \ldots \ldots \ldots \ldots \ldots$

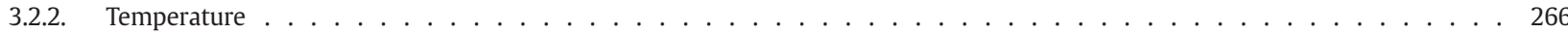

3.2.3. Redox potential . . . . . . . . . . . . . . . . . . . . . . . . . . . 267

3.2.4. Reactive species involved in reductive dechlorination reactions. . . . . . . . . . . . . . . . . . . . . 268

3.2.5. Variety of electron donors and natural sources of RM . . . . . . . . . . . . . . . . . . . . 268

4. Role of redox mediators on the reduction of nitroaromatic pollutants . . . . . . . . . . . . . . . . . . . . . . . . 269

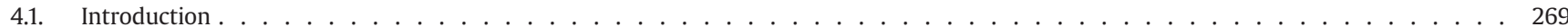

4.2. Main factors determining the input of RM in the reduction of nitroaromatic pollutants. . . . . . . . . . . . . . . . . . . . . . 269

\footnotetext{
* Corresponding author. División de Ciencias Ambientales, Instituto Potosino de Investigación Científica y Tecnológica (IPICyT), Camino a la Presa San José 2055, Col. Lomas $4{ }^{a}$. Sección, San Luis Potosí, SLP, 78216 Mexico. Tel.: +52 444 8342041; fax: +52 4448342010.

E-mail address: fjcervantes@ipicyt.edu.mx (F.J. Cervantes).
} 


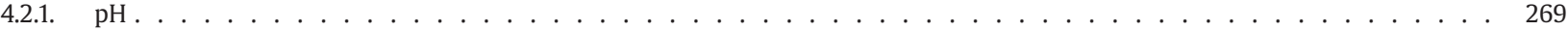

4.2.2. Redox potential. . . . . . . . . . . . . . . . . . . . . . . . . . . . . . . . . . . . 270

4.2.3. Biodegradation pathway. . . . . . . . . . . . . . . . . . . . . . . . . . . . . . . . . . . 271

5. Role of redox mediators in miscellaneous processes . . . . . . . . . . . . . . . . . . . . . . . . . . . . . 272

5.1. Introduction. . . . . . . . . . . . . . . . . . . . . . . . . . . . . . . . . . . . . . . . . 272

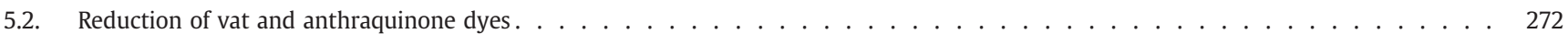

5.3. Reduction of soluble inorganic electron acceptors . . . . . . . . . . . . . . . . . . . . . . . . . . . . . . . . . . . . . . . 272

5.4. Reduction of insoluble materials (minerals and anodes) . . . . . . . . . . . . . . . . . . . . . . . . . . . . 273

5.5. Electron transfer from cathodes . . . . . . . . . . . . . . . . . . . . . . . . . . . . . . . . . . . 273

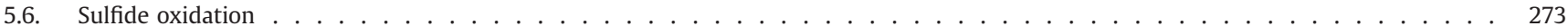

5.7. Enzymatic oxidation of organic compounds . . . . . . . . . . . . . . . . . . . . . . . . . . . . . . . . . . . . . . 273

6. Future perspectives on the application of redox mediators on the reductive (bio)transformation of contaminants . . . . . . . . . . . . . 273

6.1. Introduction. . . . . . . . . . . . . . . . . . . . . . . . . . . . . . . . . . . . . . . 273

6.2. Cost-effective sources of redox active groups . . . . . . . . . . . . . . . . . . . . . . . . . . . . . . . . . . 273

6.3. Engineered HS for their application as RM in redox reactions . . . . . . . . . . . . . . . . . . . . . . . . . . . . . . . . 274

6.4. Immobilization of RM . . . . . . . . . . . . . . . . . . . . . . . . . . . . . . . . . . . . . . . . . . . 274

6.5. Overcoming limitations during catalysis involving RM . . . . . . . . . . . . . . . . . . . . . . . . . . . . . . . . . . . . . 274

Acknowledgements . . . . . . . . . . . . . . . . . . . . . . . . . . . . . . . . . . . . . . . . . . 275

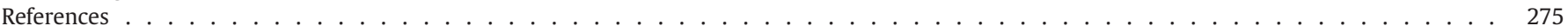

\section{Introduction}

The chemical and petrochemical industry produces worldwide thousands of compounds each year and it is still in expansion. The global market of this sector is huge and only during the first 9 months of 2004, the sales in the USA summed up US\$110.4 billion for 26 companies of the chemical sector (Storck et al., 2005). Unfortunately, linked to the great economical benefits obtained from the chemical and petrochemical production, large volumes of wastewaters containing many different toxic and recalcitrant pollutants are being discharged (Razo-Flores et al., 2006). Among the contaminants generated are electron-withdrawing compounds, such as azo dyes,

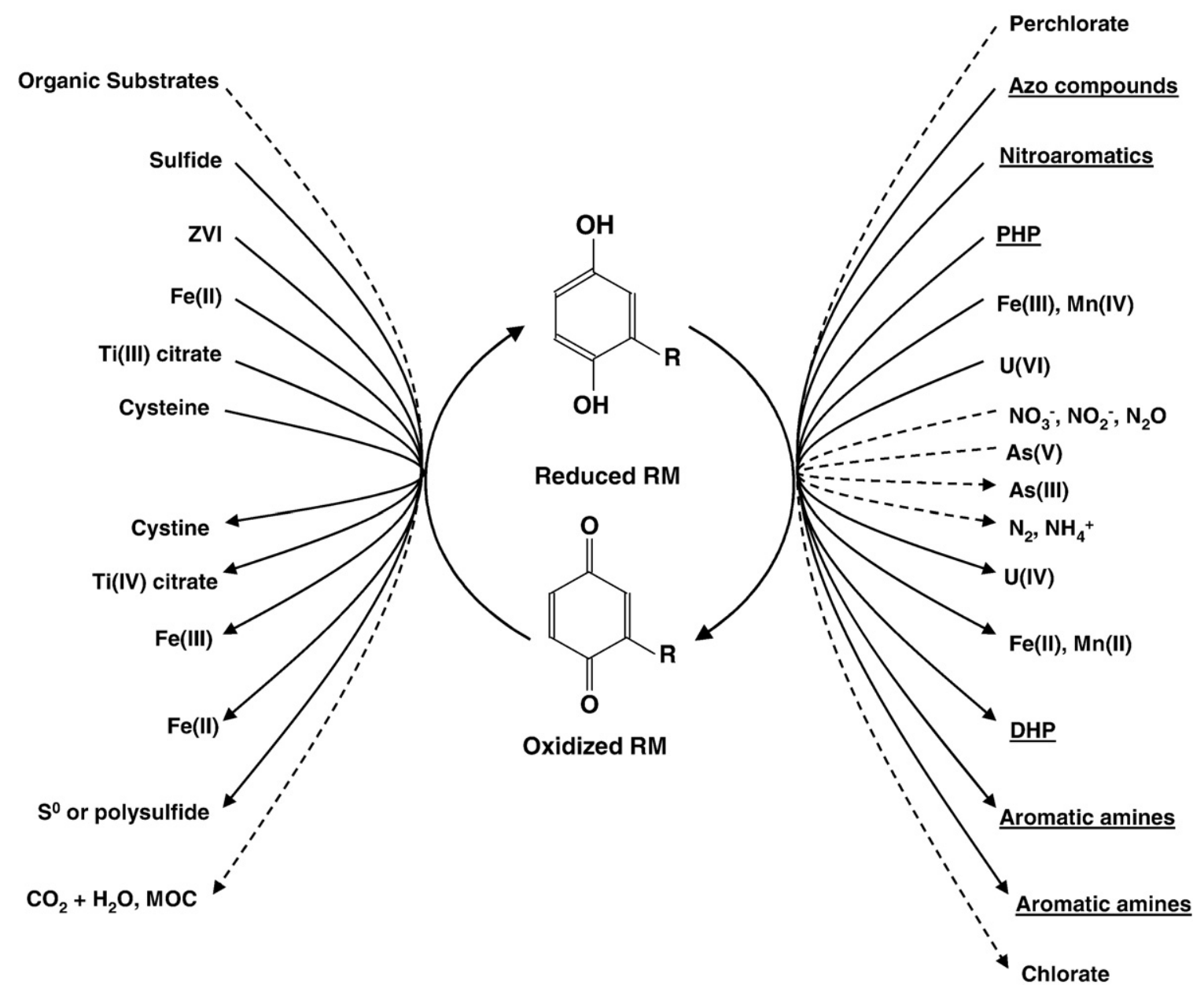

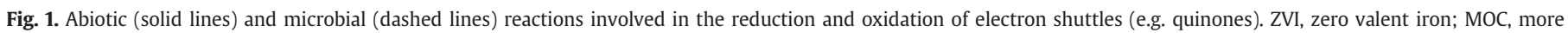
oxidized compounds; PHP, polyhalogenated pollutants; DHP, dehalogenated pollutants. 
nitroaromatics, halogenated aliphatics, halogenated aromatics and metalloids, which remain unaffected during conventional aerobic wastewater treatment. In contrast, under anaerobic conditions, these contaminants can undergo reductive transformations (Field et al., 1995). During the last two decades, evidence accumulated showing the potential to convert pollutants containing electrophilic groups in their structures, in high-rate anaerobic bioreactors, such as upflow anaerobic sludge bed (UASB) and expanded granular sludge bed (EGSB) systems (Donlon et al., 1996; Christiansen et al., 1997; RazoFlores et al., 1999; Van der Zee et al., 2001; Dos Santos et al., 2005c). However, reductive transformation of many different recalcitrant compounds proceeds very slowly due to electron transfer limitations and to toxicity effects leading to poor performance or even collapse of anaerobic bioreactors (Rodgers and Bunce, 2001; Van der Zee et al., 2001).

Since the 1990s, extensive research has been conducted to explore the catalytic effects of different electron shuttles on redox (bio) transformation processes. Electron shuttles, also referred to as redox mediators (RM), are organic molecules that can reversibly be oxidized and reduced, thereby conferring the capacity to serve as an electron carrier in multiple redox reactions. As will be explained below, RM accelerate reactions by lowering the activation energy of the total reaction. In some cases, the presence of RM may even be a prerequisite for the reaction to take place.

$\mathrm{RM}$ are capable of transferring electrons in redox reactions between a wide variety of both inorganic and organic compounds. Fig. 1 illustrates most redox reactions involved in the reduction and oxidation of RM by abiotic and biological means. Reduction of RM can be promoted by chemical reaction with reductants commonly found in many anaerobic environments (e.g. sulfide, cysteine, Fe(II)) (Schwarzenbach et al., 1990; Curtis and Reinhard, 1994) or by electron donors proposed in restoration technologies (e.g. zero-valent iron (ZVI), Ti(III) citrate) (Tratnyek et al., 2001; Dror and Schlautman, 2004; Keum and Li, 2004). Furthermore, reduction of RM can be linked to the anaerobic oxidation of organic substrates by microorganisms. The most relevant RM supporting the microbial oxidation of organic compounds under anaerobic conditions are humic substances and their quinoid analogues (Field and Cervantes, 2005). Microbial<smiles>Cc1cc2nc3c(=O)[nH]c(=O)nc-3n(CC(O)C(O)C(O)CO)c2cc1C</smiles>

riboflavi

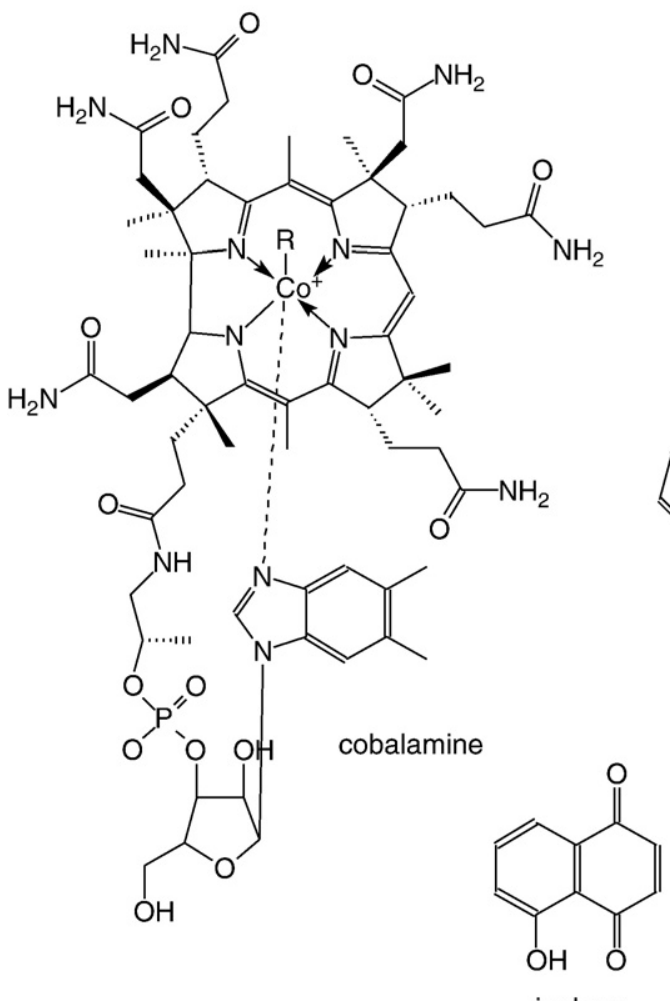

juglone<smiles>O=C1C(=O)c2ccc(S(=O)(=O)O[Na])cc2C(=O)c2ccccc21</smiles><smiles>c1ccc2nc3ccccc3nc2c1</smiles>

phenazine<smiles>Cc1cc2nc3ccc(N(C)C)cc3nc2cc1N</smiles>

Neutral Red<smiles>C[n+]1ccc(-c2cc[n+](C)cc2)cc1</smiles>

methyl viologen<smiles>O=[N+]([O-])O[Na]</smiles><smiles>O=C1C=C(O)C(=O)c2ccccc21</smiles>

lawsone<smiles>CC1=CC(=O)c2ccccc2C1=O</smiles>

menadione

Fig. 2. Structure of the main redox mediators reported in the literature. 
reduction of quinones can be linked to the anaerobic oxidation of a large variety of organic substrates including alcohols, short chain fatty acids, monosaccharides (Lovley et al., 1996; Cervantes et al., 2000a), as well as priority pollutants, such as toluene (Cervantes et al., 2001a), vinyl chloride, dichloroethene (Bradley et al., 1998) and phenolic compounds (Cervantes et al., 2000a,b). On the opposite site, several electron-withdrawing compounds accept electrons from reduced RM, thereby promoting their re-oxidation. From the environmental or public health point of view, the most relevant redox reactions involved in the re-oxidation of RM include the abiotic transfer of electrons from reduced RM towards azo dyes (Rau et al., 2002), nitroaromatics (Tratnyek and Macalady, 1989; Borch et al., 2005; Kwon and Finneran, 2006), polyhalogenated pollutants (Curtis and Reinhard, 1994; Kappler and Haderlein, 2003) and U(VI) (Fredrickson et al., 2000). In these reactions, quinones or humic materials are the most studied RM. Reduced quinones can also serve as electron donors to support the microbial reduction of perchlorate, arsenate, nitrate, nitrite and nitrous oxide (Lovley et al., 1999; Aranda-Tamaura et al., 2007).

Fig. 2 illustrates the structure of several electron shuttles reported during the redox (bio)transformation of pollutants. For a RM to be an effective electron shuttle, its standard redox potential ( $\left.E_{0}^{\prime}\right)$ should ideally be in between those of the two eventual half reactions. However, as the RM's efficacy will also depend on the activation energy of its reduction and oxidation, in comparison to that of the non-mediated total reaction, it may occur that even compounds with $E_{0}^{\prime}$ lower than the primary electron donor or higher than the terminal electron acceptor may also be effective RM.

The concept of mediated electron transfer has been known for a very long time. As early as 1931, in a study on bacterial electricity generation, it was demonstrated that electromotively active organic compounds, such as benzoquinone, or inorganic compounds, such as ferricyanide, could increase the electric current of a bacterial half-cell (Cohen, 1931). To our knowledge, the first paper mentioning a role of quinoid enzyme cofactors in the reduction of azo dyes dates from the late 1960s (Roxon et al., 1967). RM-catalyzed reduction of nitroaromatics has firstly been reported by Brown (1981). Moreover, the rate of reduction of several halogenated hydrocarbons was shown to improve with increasing the amount of natural organic matter (NOM) in sediments (Peijnenburg et al., 1992), presumably due to the catalytic input of redox active groups in NOM. Nevertheless, Tratnyek and Macalady (1989) and Curtis and Reinhard (1994) were probably the first researchers to focus their research on evidencing the input of RM on the reduction of nitroaromatics and polyhalogenated pollutants, respectively. From then on, and certainly after the publication of other thorough papers on basic aspects of the role of RM (Keck et al., 1997; Kudlich et al., 1997) and the boosting attention to microbial fuel cells (Bullen et al., 2006; Logan et al., 2006) the topic became increasingly popular, both in basic and in applied research. The application of RM for wastewater treatment and aquifer bioremediation has deserved considerable attention during the last years. The first studies focused on the application of RM on reductive (bio)transformation of contaminants were carried out during the decolorization of azo dyes in high rate UASB reactors (Cervantes et al., 2001b; Van der Zee et al., 2001).

The aim of this review paper is to summarize the results of RMcatalyzed reductive (bio)transformation processes. The article describes the impact of different RM on azo dye reduction (Section 2), reductive dehalogenation (Section 3) and reduction of nitroaromatics (Section 4). Section 5 briefly exemplifies miscellaneous redox reactions catalyzed by RM. Finally, Section 6 proposes several research areas required to improve the applicability of RM on the (bio)transformation of contaminants.

\section{Role of redox mediators on azo dye reduction}

\subsection{Introduction}

Azo dyes are aromatic structures linked together by azo (-N=N-) chromophores. They represent the largest class of dyes applied in textile-processing industries. Due to their color and possible mutagenicity, their removal from wastewater is of major concern. For that purpose, sequential anaerobic-aerobic treatment is the most logical biological strategy for the removal of azo dyes from wastewater. The dyes' electron withdrawing azo linkages, which are very recalcitrant towards oxidative reactions, can be reduced under anaerobic conditions, yielding aromatic amines. These aromatic amines are in turn likely to be removed by aerobic treatment, through a combination of biodegradation, autoxidation and adsorption (Field et al., 1995; Van der Zee and Villaverde, 2005). Anaerobic azo dye reduction has been thoroughly investigated and several review papers summarize the results, e.g. Stolz (2001) and Pandey et al. (2007). It is clear that most, if not all, azo compounds are susceptible to reductive fission and that the capacity to reduce azo dyes under anaerobic conditions is almost ubiquitous among a wide range of bacterial species. In addition, azo dyes can be chemically reduced by bulk reducing agents, such as sulfide. Consequently, anaerobic bioreactor treatment of azo dye containing wastewaters will normally lead to a certain extent of reductive decolorization. In a recent review of a large number of bioreactor studies it was concluded that the extent of anaerobic color removal in these studies was generally high (70-100\%) but that decolorization often proceeds very slowly, requiring long hydraulic residence times (Van der Zee and Villaverde, 2005). It is obvious, therefore, that accelerating azo dye reduction by electron shuttling RM will be highly relevant for the applicability of biological techniques to remove azo dyes from wastewater.

Tables 1-5 give a summary of the studies reporting the impact of $\mathrm{RM}$ on the rates of azo dye reduction in different biological and chemical systems. In the 'Results' columns of the tables, it can be seen that RM accelerated the reaction in most of the systems studied, often doubling to multiplying the reaction rates. In some cases, the presence of a RM even appeared as a prerequisite for the reaction to take place. However, the impact of RM was not always huge: sometimes the accelerating effect was small, sometimes absent, and in rare cases even adverse. This wide range of RM impacts reflects the differences between the investigated systems, their conditions, the dye/mediator/ biomass-combinations used, etc. In the following sections, the most important factors determining the impact of RM on azo dye reduction will be discussed.

\subsection{Redox mediator properties}

Several of the studies listed in Tables 1-5 report different impacts of distinct RM under otherwise similar circumstances. Part of these differences may be directly related to the $E_{0}^{\prime}$ of the shuttling compounds investigated. In addition, other physico-chemical properties of the shuttling compound may play a role in determining its feasibility and strength as a mediator for azo dye reduction.

\subsubsection{Oxidation-reduction potential}

By definition, an electron shuttling compound will only be effective as a RM for azo dye reduction if it lowers the reaction's activation energy. For that purpose, its $E_{0}^{\prime}$ should ideally be in between those of the two eventual half reactions, the reduction of an azo dye and the oxidation of a primary electron donor:

- A mediator's $E_{0}^{\prime}$ should not be much lower (ideally higher or less negative) than that of the biological reducing system (typically $-320 \mathrm{mV}$, representing the $E_{0}^{\prime}$ of $\mathrm{NAD}(\mathrm{P}) \mathrm{H}$, the cofactor with the lowest oxidation-reduction potential) or the bulk reductant (e.g. $-270 \mathrm{mV}$ in case of sulfide) - otherwise it will not be reduced sufficiently. Hence the observed failure of cyanocobalamin (Dos Santos et al., 2004a) and ethyl viologen (Kudlich et al., 1997) to act as a mediator is most probably due to their too low $E_{0}^{\prime}$ values: -530 and $-480 \mathrm{mV}$, respectively. Methyl viologen $\left(E_{0}^{\prime}=-440 \mathrm{mV}\right)$ was the most reduced shuttling compound with clear ability to act as a RM 
for azo dye reduction. It modestly increased the rate of Acid Red 27 reduction by Sphingomonas (Kudlich et al., 1997) but its impact on the reduction of Acid Yellow 23 by Bacteroides was huge, a 4.5-fold increase of the reaction rate, similar or even better than that of mediators with a higher $E_{0}^{\prime}$ (Chung et al., 1978). Especially the latter result is remarkable, since methyl viologen's $E_{0}^{\prime}$ is considerably lower than the indicated boundary level of $-320 \mathrm{mV}$.
- Likewise, a mediator's $E_{0}^{\prime}$ should not be much higher (ideally lower or more negative) than that of the azo dye - otherwise it will not reduce the azo linkage at sufficient rates. Since $E_{0}^{\prime}$-values for azo dyes, which will be unique for each specific compound, are not available, it is less easy to estimate top boundary values for a mediator's $E_{0}^{\prime}$. Polarographically determined half-wave standard potentials $\left(E_{1 / 2}\right)$ give an indication. However, reported $E_{1 / 2}$-values

Table 1

Impact of redox mediators on the reductive decolorization of azo dyes by pure cultures of different microbial strains

\begin{tabular}{|c|c|c|c|c|c|c|}
\hline Azo Dye ${ }^{a}$ & Microorganism(s) ${ }^{\mathrm{b}}$ & $\begin{array}{l}\text { Electron } \\
\text { donor }\end{array}$ & Mediator $^{\mathrm{C}}$ & $\begin{array}{l}\mathrm{RM} / \\
\mathrm{AD}^{- \text {ratio }^{\mathrm{d}}}\end{array}$ & Results $^{\mathrm{e}}$ & Reference \\
\hline Acid Yellow 23 & $\begin{array}{l}\text { Proteus vulgaris ( } 0-74 \text { h starved } \\
\text { cells) }\end{array}$ & Glucose & RF & $\begin{array}{l}0.0025- \\
0.0175\end{array}$ & Max. $10 \times$; no additional effect RM at RM/AD $>0.012$ & $\begin{array}{l}\text { Roxon } \\
\text { et al. (1967) }\end{array}$ \\
\hline Acid Yellow 23 & Bacteroides thetaitaomicron & Glucose & $\begin{array}{l}\text { MV, BV, CV, } \\
\text { NR, PS FAD, } \\
\text { MQ, FMN, JGB }\end{array}$ & 0.0125 & $\begin{array}{l}\text { MV, BV, CV, NR: } 4.5 \times \text {; PS, FAD: } 3.5 \times \text {; MQ, FMN: } 3 \times \text {; } \\
\text { JGB: } 2.5 \times\end{array}$ & $\begin{array}{l}\text { Chung } \\
\text { et al. (1978) }\end{array}$ \\
\hline Poly-T Poly-S & $\begin{array}{l}18 \text { pure cultures of anaerobic (10) } \\
\text { and facultative ( } 8 \text { ) } \text { bacteria }^{\mathrm{f}}\end{array}$ & Broth & FMN & $0.0119^{\mathrm{g}}$ & $\begin{array}{l}\text { Stimulatory effect depends on the dye-organism } \\
\text { combination: max. } 25 \times(\text { avg. } 4.6 \times) \text { and max. } 2.3 \times \\
(\text { avg. } 1.8 \times) \text { for Poly-T and Poly-S, resp. }\end{array}$ & Brown (1981) \\
\hline Acid Red 27 & Sphingomonas xenophaga BN6 & Glucose & 2-NS-metab. & $(1)^{\mathrm{h}}$ & $6 \times$ & $\begin{array}{l}\text { Keck et al. } \\
(1997,2002)\end{array}$ \\
\hline Acid Red 27 & $\begin{array}{l}\text { Sphingomonas paucimobilis; } \\
\text { E. coli JM109 }\end{array}$ & Glucose & 2-NS-metab. & $(1)^{\mathrm{h}}$ & Increase (not quantified) & $\begin{array}{l}\text { Keck et al. } \\
\text { (1997) }\end{array}$ \\
\hline Acid Red 27 & Sphingomonas sp. strain BN6 & Glucose & $\begin{array}{l}\text { AQS, LAW, } \\
\text { AQDS, BV, } \\
\text { MV, FAD, } \\
\text { (EV) }\end{array}$ & 0.2 & $\begin{array}{l}\text { AQS: } 10.5 \times \text {; LAW: } 10 \times \text {; AQDS: } 6.5 \times \text {; BV: } 2 \times \text {; MV, } \\
\text { FAD: } 1.5 \times \text {; EV: no effect }\end{array}$ & $\begin{array}{l}\text { Kudlich } \\
\text { et al. (1997) }\end{array}$ \\
\hline Acid Red 27 & Sphingomonas sp. strain BN6 & Glucose & AQS & Max. 3 & $\begin{array}{l}\text { Without RM: negligible reduction; AQS: increase to } \\
2.3 \mu \mathrm{mol} \mathrm{min}^{-1} \mathrm{~g}^{-1} \text { protein; no additional effect RM at } \\
\mathrm{RM} / \mathrm{AD}>\sim 1.4\end{array}$ & $\begin{array}{l}\text { Kudlich } \\
\text { et al. (1997) }\end{array}$ \\
\hline $\begin{array}{l}\text { Acid Red } 27 \\
\text { Acid Red } 1 \\
\text { Food Yellow } 3 \\
\text { Acid Black } 1\end{array}$ & Sphingomonas sp. strain BN6 & Glucose & AQS & Max. 0.6 & $\begin{array}{l}\text { Without RM: negligible reduction; increase to max. } 4.5 \text {, } \\
\text { 2.2, } 6.6 \text { and } 3.6 \mu \mathrm{mol} \mathrm{min}^{-1} \mathrm{~g}^{-1} \text { protein (AR27, AR1, FY3 } \\
\text { and } \mathrm{AB} 1 \text {, resp.) }\end{array}$ & $\begin{array}{l}\text { Kudlich } \\
\text { et al. (1997) }\end{array}$ \\
\hline DHAB & Bacteroides fragilis & Broth & BV & 0.17 & Increase (not quantified) & $\begin{array}{l}\text { Bragger } \\
\text { et al. (1997) }\end{array}$ \\
\hline Acid Red 27 & Clostridium perfringens & Glucose & RF, BV & 4.2 & RF: $\sim 2.5 \times, B V: \sim 1.6 \times$ & $\begin{array}{l}\text { Semdé et al. } \\
\text { (1998) }\end{array}$ \\
\hline Acid Red 27 & $\begin{array}{l}10 \text { pure cultures from distinct } \\
\text { taxonomic groups }{ }^{\mathrm{i}}\end{array}$ & Glucose & $\begin{array}{l}\text { AQS, AQDS, } \\
\text { LAW, LAP } \\
\text { PLUM }\end{array}$ & 2.5 & $\begin{array}{l}\text { Stimulatory effect varies largely between different } \\
\text { organism-RM combinations: average effect from } \\
0.1-0.5 \text { to } 2-10 \mu \mathrm{mol} \text { dye } \mathrm{min}^{-1} \mathrm{~g}^{-1} \text { protein; One } \\
\text { species, } \text { H. salinarum, does not reduce the dye at all. }\end{array}$ & $\begin{array}{l}\text { Rau et al. } \\
\text { (2002) }\end{array}$ \\
\hline $\begin{array}{l}\text { Acid Red } 27 \\
\text { Acid Orange } 20 \\
\text { Acid Orange } 7 \\
\text { Food Yellow } 3 \\
\text { Acid Red } 18 \\
\text { Food Red } 17 \\
\text { Acid Red } 14 \\
\text { Acid Red } 1 \\
\text { Acid Yellow } 23 \\
\text { Acid Black } 1 \\
\text { Food Black } 1\end{array}$ & $\begin{array}{l}\text { Sphingomonas xenophaga } \\
\text { BN6; Escherichia coli } \mathrm{K} 12\end{array}$ & Glucose & AQS, LAW & 2.5 & $\begin{array}{l}\text { Stimulatory effect varies largely between different } \\
\text { dye-organism-RM combinations: Average effect } 26 \times \text {; } \text {. } \\
\text { xenophaga: AQS about } 4 \times \text { more effective than LAW; E. coli: } \\
\text { LAW about } 37 \times \text { more effective than AQDS }\end{array}$ & $\begin{array}{l}\text { Rau et al. } \\
\text { (2002) }\end{array}$ \\
\hline Reactive Black 5 & Shewanella strain J18 143 & Formate & AQDS & 4 & $3 \times$ & $\begin{array}{l}\text { Pearce et al. } \\
(2006)\end{array}$ \\
\hline Acid Red 27 & Shewanella decolorationis S12 & Formate & AQS, AQDS & $\begin{array}{l}1-5 \\
1-12\end{array}$ & $\begin{array}{l}\text { AQS: } 1.75 \times \text { at. } R M / A D=1-2 \text {; relative impact of } R M \\
\text { decreases and becomes even adverse at higher RM/AD. } \\
\text { AQDS: } 1.33 \times \text { at } R M / A D=1 \text {; decreasing impact, to none, } \\
\text { at higher } \mathrm{RM} / \mathrm{AD} \text {. }\end{array}$ & $\begin{array}{l}\text { Hong et al. } \\
(2007)\end{array}$ \\
\hline $\begin{array}{l}\text { Acid Red } 73 \\
\text { Acid Yellow } 36 \\
\text { Acid Orange } 10 \\
\text { Acid Orange } 7 \\
\text { Acid Red } 26\end{array}$ & Shewanella decolorationis S12 & Formate & AQS, AQDS & $\begin{array}{l}1-5 \\
1-12\end{array}$ & $\begin{array}{l}\text { Without RM, CRE(12 h) 19\%; AQS: at RM/AD=1, CRE(12 h) } \\
\sim 96 \% \text {; no additional effect at RM/AD =3; adverse effect at } \\
\text { RM/AD =5; AQDS: at RM/AD =3 and 6, CRE(12 h) } \sim 95 \% \text {; no } \\
\text { additional effect at higher RM/AD }\end{array}$ & $\begin{array}{l}\text { Hong et al. } \\
(2007)\end{array}$ \\
\hline Reactive Red 2 & $\begin{array}{l}\text { Methanothermobacter } \\
\text { thermoautotrophicus } \Delta \mathrm{H}\left(65{ }^{\circ} \mathrm{C}\right) \text {; } \\
\text { Methanothermobacter related strain } \\
\mathrm{NJ} 1\left(55^{\circ} \mathrm{C}\right) \text {; Methanosarcina barkeri } \\
\left(37^{\circ} \mathrm{C}\right)\end{array}$ & $\mathrm{H}_{2}$ & $\mathrm{RF}$ & 0.12 & $\begin{array}{l}\text { Thermophilic strains: only dye reduction in the presence } \\
\text { of RF mesophilic strain: RF accelerates dye reduction }\end{array}$ & $\begin{array}{l}\text { Dos Santos } \\
\text { et al. (2006) }\end{array}$ \\
\hline $\begin{array}{l}\text { Pigment } \\
\quad \text { Orange } 5^{j}\end{array}$ & Shewanella strain J18 143 & Formate & AQDS & 2 & $\sim 4 \times\left(30^{\circ} \mathrm{C}\right)$ & $\begin{array}{l}\text { Pearce et al. } \\
(2008)\end{array}$ \\
\hline $\begin{array}{l}\text { Acid Orange } 52 \\
\text { 'Dye I' } \\
\text { Acid Orange } 7 \\
\text { 'Dye III' }\end{array}$ & Issatchenkia occidentalis (yeast) ${ }^{\mathrm{k}}$ & Glucose & AQS & Max. 9.5 & $\begin{array}{l}\text { AO52: max. } 3 \times \text {; no additional effect RM at RM/AD }>2.1 \text {; } \\
\text { 'Dye I': similar results as with AO52; AO7 and 'Dye III': } \\
\text { adverse effect of RM (not quantified) }\end{array}$ & $\begin{array}{l}\text { Ramalho } \\
\text { et al. 2004) }\end{array}$ \\
\hline
\end{tabular}


Table 2

Impact of redox mediators on the reductive decolorization of azo dyes by cell-free enzymatic extracts

\begin{tabular}{|c|c|c|c|c|c|}
\hline Azo dye $\mathrm{a}^{\mathrm{a}}$ & System & Mediator ${ }^{\mathrm{b}}$ & RM/AD-ratio ${ }^{c}$ & Results $^{\mathrm{d}}$ & Reference \\
\hline Acid Yellow 23 & $\begin{array}{l}\text { Crude enzyme preparations } \\
\text { (supernatant fraction) } \\
\text { of Proteus vulgaris }+ \\
\text { NADPH-generating } \\
\text { system }\end{array}$ & RF, FMN, FAD & Max. 0.536 & RF: max. $14 \times$; FMN: max. $16 \times$; FAD: $\max .3 \times$ & Roxon et al. (1967) \\
\hline Acid Yellow 9 & $\begin{array}{l}\text { Cell extracts (supernatant fraction) of } \\
\text { Streptococcus faecalis + } \\
\text { NADH-generating system }\end{array}$ & RF, FMN, FAD & 0.0033 & RF: $1.8-3.6 \times ;$ FMN: $1.5-3.1 \times ;$ FAD: $1.4-2.4 \times$ & Scheline and Nygaard (1970) \\
\hline Acid Red 1 & $\begin{array}{l}\text { Cell-free extracts of Streptococcus faecalis + } \\
\text { NADH-generating system }\end{array}$ & RF, FMN, FAD & Max. 1.34 & $\begin{array}{l}\text { Without RM negligible reduction; } \\
\text { with } \mathrm{RM} \text { increase to } 2.7,2.3 \text { and } \\
2.0 \mu \mathrm{mol} \mathrm{h} \mathrm{h}^{-1} \mathrm{mg}^{-1} \text { protein ( } \mathrm{RF} \text {, } \\
\text { FMN, FAD, respectively.); } \\
\text { very limited additional effect RM } \\
\text { at } \mathrm{RM} / \mathrm{AD}>0.4\end{array}$ & Walker et al. (1971) \\
\hline $\begin{array}{l}\text { Food Yellow } 3 \\
\text { Acid Yellow } 23 \\
\text { Poly-T, Poly-S }\end{array}$ & $\begin{array}{l}\text { Cell free extracts of rat } \\
\text { cecal bacteria + NADPH-generating } \\
\text { system }\end{array}$ & BV & $2.1-2.3^{\mathrm{e}}$ & $\begin{array}{l}50 \times(\text { FY3 }), 164 \times(\text { AY236 }), 123 \times(\text { Poly-T }) \\
118 \times(\text { Poly-S })\end{array}$ & Brown (1981) \\
\hline
\end{tabular}

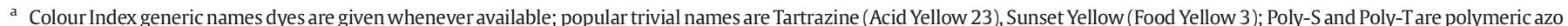
dyes based on Food Yellow 3 and Acid Yellow 23, respectively.

b $B V=$ Benzyl viologen, FAD = Flavin adenine dinucleotide, FMN= Flavin mononucleotide, RF= Riboflavin.

c RM/AD-ratio=molar ratio redox mediator: azo dye (unless otherwise stated).

d ' $x$ ' expresses the $x$-fold increase of zero-order reduction rates as compared to the RM-free controls; avg.=average.

e RM/AD-ratio as moles mediator per mol (eq) dye monomer.

have a broad range (from -530 to $-180 \mathrm{mV}$ ) and the differences between values reported for the same azo dye can vary as much as 200 mV (Dubin and Wright, 1975; Bragger et al., 1997; Semdé et al., 1998). Another and probably better indication was the result of an experiment in which the reduction of the azo dye Acid Red 27 by a large number of chemically reduced quinoid shuttling compounds with different $E_{0}^{\prime}$ values was investigated. This experiment demonstrated that this particular dye, which has an $E_{1 / 2}$ of around -250 mV (Dubin and Wright, 1975; Semdé et al., 1998) or around $-350 \mathrm{mV}$ (Bragger et al., 1997), could only be reduced by quinones with $E_{0}^{\prime}$ values below $-50 \mathrm{mV}$ (Rau et al., 2002). For other azo dyes, this threshold value, which reflects the dye's $E_{0}^{\prime}$ in combination with the reaction's activation energy, may be different, but it is reasonable to assume that the observed failure of 1,4-Benzoquinone to act as a mediator (Dos Santos et al., 2005c) and the poor impact reported for Menadione (Chung et al., 1978; Albuquerque et al., 2005) are most probably due to their too high $E_{0}^{\prime}$-values: +263 and $-19 \mathrm{mV}$, respectively.

- In the $E_{0}^{\prime}$ range between these boundaries, there will be two opposing trends with respect to a mediator's $E_{0}^{\prime}$ and its impact on the rate of azo dye reduction. In the second step of mediated azo dye reduction, i.e. the chemical reaction between the reduced mediator and the azo dye, the reaction rate will increase with decreasing $E_{0}^{\prime}$ of the mediator, as has been demonstrated with the reduction of Acid Red 27 by a series of chemically reduced quinones with different $E_{0}^{\prime}$ values (Rau et al., 2002). In the first step of mediated azo dye reduction, i.e. reduction of the mediator, an opposite trend, an increase of the reaction rate with increasing $E_{0}^{\prime}$ of the mediator, can be expected. The rate-limiting step of mediated azo dye reduction will thus be influenced by the mediator's $E_{0}^{\prime}$, with either the reduction of the dye or that of the mediator being rate-limiting, at higher and lower $E_{0}^{\prime}$ values, respectively. At midrange, combination of both trends may reveal the optimum mediator's $E_{0}^{\prime}$ (unique for each dye/reducing system combination), where both reaction steps proceed at equal rate. However, in biological systems (Tables 1-4), such an optimum $E_{0}^{\prime}$ could not be confirmed: studies with multiple redox mediators under otherwise similar conditions did not show any relationship between a mediator's $E_{0}$ and the rate of azo dye reduction (Chung et al., 1978; Kudlich et al., 1997; Rau et al., 2002), and mediators with identical $E_{0}^{\prime}$ (FAD, FMN and AQS) often had largely different impacts. For instance, Kudlich et al. (1997) observed that Acid Red 27 reduction by Sphingomonas BN6 was stimulated to a decreasing extent by the following mediators (values between brackets show $E_{0}^{\prime}$

\footnotetext{
Notes to Table 1:

a Colour Index generic names dyes are given whenever available; popular trivial names are Naphthol Blue Black (Acid Black 1), Amaranth (Acid Red 27), Tartrazine (Acid Yellow 23),

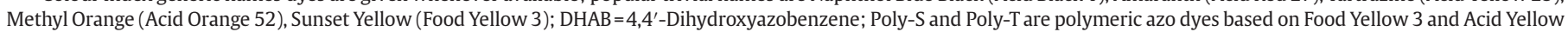
23, respectively; 'Dye I' = m-[(4-dimethylamino)phenylazo]benzenesulfonic acid, 'Dye III' = m-[(2-hydroxy-1-napthyl)azo]benzenesulfonic acid.

b Although culture conditions vary between studies, anaerobic conditions prevailed when reducing azo dyes (unless otherwise stated).

c $\mathrm{AQDS}=9,10$-Anthraquinone-2,6-disulfonate, $\mathrm{AQS}=9,10$-Anthraquinone-2-sulfonate, $\mathrm{BV}=\mathrm{Benzyl}$ viologen, $\mathrm{CV}=\mathrm{Crystal} \mathrm{Violet}, \mathrm{EV}=\mathrm{Ethyl}$ viologen, $\mathrm{FAD}=\mathrm{Flavin}$ adenine dinucleotide,

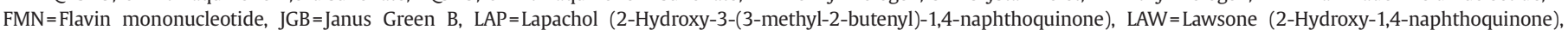

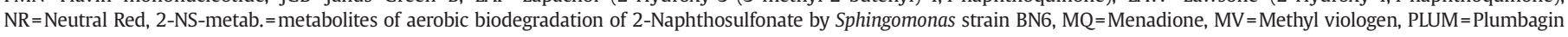
(5-Hydroxy-2-methyl-1,4-naphthoquinone), PS=Phenosafranin, RF= Riboflavin.

d RM/AD-ratio= molar ratio redox mediator: azo dye (unless otherwise stated).

e ' $x$ ' expresses the $\times$-fold increase of zero-order reduction rates as compared to the RM-free controls; avg.= average.; resp. = respectively; CRE= color removal efficiency; dye names are logically abbreviated.

f Anaerobes: Clostridium ramosum; Clostridium paraputrificum; Clostridium sporogenes; Lactobacillus catenaforme; Acidaminococcus fermentans; Bacteroides fragilis; Ruminococcus

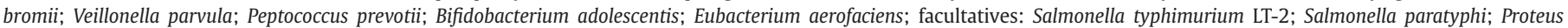
vulgaris; Klebsiella pneumoniae; Pseudomonas aeruginosa; Salmonella typhimurium TA1537; Streptococcus faecium.

g RM/AD-ratio as moles mediator per mol (eq) dye monomer.

h The original 2-NS-concentration was $0.5 \mathrm{mM}$, equal to the dye concentration subsequentially applied.

${ }^{i} \alpha$-Protebacteria: Sphingomonas xenophaga BN6 and Agrobacteria tumefaciens; $\beta$-Proteobacteria: Ralstonia eutropha 335 and Hydrogenophaga palleronii; $\gamma$-Proteobacteria: Escherichia

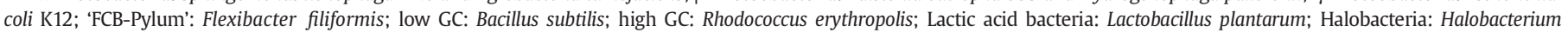
salinarum.

j As commercial dispersion GemSperse Orange EX5.

${ }^{\mathrm{k}}$ Microaerobic conditions (shaking flasks exposed to air), albeit with soluble oxygen concentrations below detection level when azo dye reduction took place.
} 
Table 3

Impact of redox mediators on the reductive decolorization of azo dyes by mixed microbial cultures under batch, anaerobic conditions

\begin{tabular}{|c|c|c|c|c|c|c|}
\hline Azo dye ${ }^{a}$ & System $^{\mathrm{b}}$ & $\begin{array}{l}\text { Electron } \\
\text { donor }^{c}\end{array}$ & Mediator $^{\mathrm{d}}$ & $\begin{array}{l}\mathrm{RM} / \mathrm{AD}- \\
\text { ratio }^{\mathrm{e}}\end{array}$ & Results $^{f}$ & Reference \\
\hline Acid Red 27 & AAS & Glucose & AQS & Max. 3 & $\begin{array}{l}0 \rightarrow 3 \mu \mathrm{mol} /(\min . \mathrm{g} \text { protein); no additional } \\
\text { effect at } \mathrm{RM} / \mathrm{AD}>\sim 1.2\end{array}$ & $\begin{array}{l}\text { Kudlich et al. } \\
\text { (1997) }\end{array}$ \\
\hline Acid Red 27 & AAS & Glucose & $\begin{array}{l}\text { AQS, LAW, } \\
\text { Henna } \\
\text { leaves }^{g}\end{array}$ & $\begin{array}{l}\text { Max. } 2 \\
\max .6 \mathrm{~g} \\
\operatorname{mmol}^{-1}\end{array}$ & $\begin{array}{l}\text { AQS: max. } 10-15 \times \text {; LAW: max. } 7-12 \times \text {; } \\
\text { Henna leaves: } \operatorname{max.~} 9 \times(\text { z.o. })\end{array}$ & $\begin{array}{l}\text { Rau et al. } \\
(2002)\end{array}$ \\
\hline Reactive Orange 14 & AGS & Glucose & $\mathrm{RF}$ & 0.13 & $1.5-2 \times$ & $\begin{array}{l}\text { Cervantes et al. } \\
\text { (2006) }\end{array}$ \\
\hline $\begin{array}{l}\text { Reactive Orange } 14 \\
\text { Direct Blue } 53 \\
\text { Direct Blue } 71\end{array}$ & AGS & Glucose & $\begin{array}{l}\text { AQDS, LAW, } \\
\text { RF }\end{array}$ & 0.05 & $\begin{array}{l}\text { Stimulatory effect varies largely between } \\
\text { different dye-RM combinations: avg. } 2 \times \text {; max. } 3.8 \times\end{array}$ & $\begin{array}{l}\text { Encinas- } \\
\text { Yocupicio et al. } \\
\text { (2006) }\end{array}$ \\
\hline Acid Orange 52 & AGS & Glucose & AD-metab. & $0.04^{\mathrm{h}}$ & $\sim 2 \times$ & $\begin{array}{l}\text { Encinas- } \\
\text { Yocupicio et al. } \\
\text { (2006) }\end{array}$ \\
\hline Reactive Red 2 & AGS & VFA & AQDS & Max. 1.56 & Max. $7 \times$ & $\begin{array}{l}\text { Van der Zee } \\
\text { et al. (2001) }\end{array}$ \\
\hline Mordant Yellow 10 & AGS & VFA & $\mathrm{RF}$ & Max. 0.11 & Max. 2 × (z.o.) & $\begin{array}{l}\text { Field and Brady } \\
\text { (2003) }\end{array}$ \\
\hline $\begin{array}{l}\text { Hydrolyzed Reactive } \\
\quad \text { Red } 2\end{array}$ & AGS at $55{ }^{\circ} \mathrm{C}$ & $\begin{array}{l}\text { VFA, Glucose/ } \\
\text { VFA, Glucose, } \\
\mathrm{H}_{2}\end{array}$ & AQDS & 0.04 & $\begin{array}{l}\text { VFA: } 1.4 \times ; \text { Glucose/VFA; } 1.6 \times \text {, Glucose: } \\
2.3 \times ; \mathrm{H}_{2}: 2.4 \mathrm{x}\end{array}$ & $\begin{array}{l}\text { Dos Santos et al. } \\
\text { (2003) }\end{array}$ \\
\hline $\begin{array}{l}\text { Hydrolyzed Reactive } \\
\quad \text { Red } 2\end{array}$ & AGS at different $T$ & $\begin{array}{l}\text { VFA, Glucose/ } \\
\text { VFA }\end{array}$ & AQDS & 0.4 & $\begin{array}{l}\text { VFA: } 1.7 \times, 1.9 \times, 1.9 \times, 1.6 \times, 1.7 \times \text {, at } 45,55,60,65 \\
\text { and } 75{ }^{\circ} \mathrm{C} \text {, resp.; Glucose/VFA: } 2.0 \times, 2.6 \times, 2.4 \times, 2.1 \times \\
\text { and } 1.5 \times \text {, at } 45,55,60,65 \text { and } 75^{\circ} \mathrm{C} \text {, resp. }\end{array}$ & $\begin{array}{l}\text { Dos Santos et al. } \\
\text { (2003) }\end{array}$ \\
\hline $\begin{array}{l}\text { Reactive Red } 2 \\
\text { Acid Orange } 7 \\
\text { Mordant Yellow } 10\end{array}$ & AGS at $55{ }^{\circ} \mathrm{C}$ & Glucose/VFA & $\begin{array}{l}\text { AQDS, AQS, } \\
\text { RF, (CNB12) }\end{array}$ & 0.04 & $\begin{array}{l}\text { Stimulatory effect varies largely between different } \\
\text { dye-RM combinations: avg. } 1.7 \times(\text { RR2 and AO7) or } 1.5 \times \\
\text { (z.o., MY10); CNB12 not effective }\end{array}$ & $\begin{array}{l}\text { Dos Santos et al. } \\
\text { (2004a) }\end{array}$ \\
\hline $\begin{array}{l}\text { Hydrolyzed Reactive } \\
\quad \text { Red2 }\end{array}$ & AGS at different $T$ & Glucose/VFA & AQDS & 0.04 & $\begin{array}{l}30{ }^{\circ} \mathrm{C}: 5 \times ; 5{ }^{\circ} \mathrm{C}: 1.5 \times \text {; } \mathrm{k} \text { of RM-free control is } 6 \times \\
\text { higher at } 55{ }^{\circ} \mathrm{C} \text { as compared to } 30{ }^{\circ} \mathrm{C}\end{array}$ & $\begin{array}{l}\text { Dos Santos et al. } \\
\text { (2004b) }\end{array}$ \\
\hline $\begin{array}{l}\text { Hydrolyzed Reactive } \\
\quad \text { Red2 }\end{array}$ & AGS at $55{ }^{\circ} \mathrm{C}$ & Glucose/VFA & AQDS & 0.04 & $1.4-1.7-x$ & $\begin{array}{l}\text { Dos Santos et al. } \\
\text { (2004b) }\end{array}$ \\
\hline $\begin{array}{l}\text { Hydrolyzed Reactive } \\
\quad \text { Red2 }\end{array}$ & AGS at $55{ }^{\circ} \mathrm{C}$ & Glucose/VFA & AQDS & Max. 33 & Max. $5.9 \times$; hardly any additional effect at RM/AD $>4$ & $\begin{array}{l}\text { Dos Santos et al. } \\
\text { (2004b) }\end{array}$ \\
\hline $\begin{array}{l}\text { Hydrolyzed Reactive } \\
\quad \text { Red } 2\end{array}$ & AGS at different $T$ & Glucose & $\begin{array}{l}\mathrm{AQS},(\mathrm{BQ}) \\
(\mathrm{AQS}+\mathrm{BQ})\end{array}$ & 0.17 & $\begin{array}{l}\text { AQS: } 3.8 \times \text { and } 2.3 \times \text {, at } 30 \text { and } 55{ }^{\circ} \mathrm{C} \text {, resp.; BQ: slight } \\
\text { stimulation at } 30{ }^{\circ} \mathrm{C} \text {, slight adversary effect at } 55^{\circ} \mathrm{C} \text {; } \\
\text { AQS+BQ: no effect; } k \text { of RM-free control is } 5.6 \times \\
\text { higher at } 55^{\circ} \mathrm{C} \text { as compared to } 30{ }^{\circ} \mathrm{C}\end{array}$ & $\begin{array}{l}\text { Dos Santos et al. } \\
\text { (2005b) }\end{array}$ \\
\hline $\begin{array}{l}\text { Reactive Red } 2 \\
\text { Reactive Orange } 14\end{array}$ & $\begin{array}{l}\text { AGS at different } T, \text { w } \\
\text { or w/o BES or } \\
\text { vancomycin }\end{array}$ & Glucose & $\mathrm{RF}$ & 0.04 & $\begin{array}{l}30{ }^{\circ} \mathrm{C}: 2.4 \times \text { and } 2.2 \mathrm{x} ; 55{ }^{\circ} \mathrm{C}: 1.7 \times \text { and } 2.9 \times, \mathrm{RR} 2 \text { and } \\
\text { RO14, resp.; } k \text { of } \mathrm{RM} \text {-free control is } 3.7 \text { and } 4.4 \times \text { higher } \\
\text { at } 55{ }^{\circ} \mathrm{C} \text { than at } 30{ }^{\circ} \mathrm{C}, \mathrm{RR} 2 \text { and } \mathrm{RO} 14 \text {, resp.; BES hardly } \\
\text { affects dye reduction (w or w/o RM); vancomycin strongly } \\
\text { affects dye reduction (w or w/o RM) }\end{array}$ & $\begin{array}{l}\text { Dos Santos et al. } \\
\text { (2005a) }\end{array}$ \\
\hline Reactive Red 2 & $\begin{array}{l}\text { AGS at different } T, w \\
\text { or w/o BES or } \\
\text { vancomycin }\end{array}$ & $\begin{array}{l}\text { Acetate, } \mathrm{H}_{2} \text {, } \\
\text { formate, } \\
\text { methanol }\end{array}$ & $\mathrm{RF}$ & 0.04 & $\begin{array}{l}\text { Acetate: } 3.7 \times \text { and } 1.5 \times \text {; } \mathrm{H}_{2}: 4.6 \times \text { and } 3.8 \times \text {; formate: } 2.1 \times \\
\text { and } 2.0 \times \text {; methanol: } 2.8 \times \text { and } 2.4 \times\left(30 \text { and } 55^{\circ} \mathrm{C} \text {, respectively); }\right. \\
\text { w or w/o RM, BES stimulates azo dye reduction with } \mathrm{H}_{2} \text { and } \\
\text { formate but has no effect with acetate or methanol }\end{array}$ & $\begin{array}{l}\text { Dos Santos et al. } \\
\text { (2005a) }\end{array}$ \\
\hline Reactive Red 2 & $\begin{array}{l}\text { Crushed AGS at } 30{ }^{\circ} \mathrm{C} \text {, } \\
\text { w or w/o BES }\end{array}$ & $\mathrm{H}_{2}$ & $\mathrm{RF}$ & 0.04 & $5.3 \times$; BES stimulates azo dye reduction w or w/o RM & $\begin{array}{l}\text { Dos Santos et al. } \\
\text { (2005a) }\end{array}$ \\
\hline Reactive Red 2 & AGS at different $T$ & Glucose & $\mathrm{RF}$ & Max. 0.67 & $\begin{array}{l}\text { Max. } 23 \times \text { and } 6 \times \text { at } 30 \text { and } 55^{\circ} \mathrm{C} \text {, resp.; } \\
\text { At RM/Dye }>0.17: k\left(30{ }^{\circ} \mathrm{C}\right)=k\left(55^{\circ} \mathrm{C}\right)\end{array}$ & $\begin{array}{l}\text { Dos Santos et al. } \\
\text { (2005a) }\end{array}$ \\
\hline $\begin{array}{l}\text { Reactive Red } 2 \\
\text { Reactive Red } 4 \\
\text { Reactive Orange } 14\end{array}$ & AGS at $55{ }^{\circ} \mathrm{C}$ & Glucose & $\mathrm{RF}$ & 0.04 & $\begin{array}{l}\text { RR2: } 1.7 \times \text {; RR4: no stimulation; RO14: } 2.9 \times \text {; BES hardly } \\
\text { affects dye reduction ( } w \text { or } w / 0 ~ R M) ; \text { vancomycin strongly } \\
\text { affects dye reduction ( } w \text { or } w / 0 ~ R M)\end{array}$ & $\begin{array}{l}\text { Dos Santos et al. } \\
\text { (2006) }\end{array}$ \\
\hline Reactive Orange 14 & AGS at $55{ }^{\circ} \mathrm{C}$ & $\begin{array}{l}\text { Glucose at } \\
\text { different conc. }\end{array}$ & $\mathrm{RF}$ & 0.04 & $\begin{array}{l}1.4-4.0 \times \text { : impact RM increases with increasing } \\
\text { glucose concentrations }\end{array}$ & $\begin{array}{l}\text { Dos Santos et al. } \\
\text { (2006) }\end{array}$ \\
\hline $\begin{array}{l}\text { Reactive Red } 2 \\
\quad \text { Reactive Orange } 14\end{array}$ & AGS at $55{ }^{\circ} \mathrm{C}$ & $\begin{array}{l}\text { Acetate, } \mathrm{H}_{2} \\
\text { Formate, } \\
\text { Methanol }\end{array}$ & $\mathrm{RF}$ & 0.04 & $\begin{array}{l}\text { Acetate: } 1.5 \times \text { and } 4.6 \times, \mathrm{H}_{2}: 3.8 \times-7.1 \times(\mathrm{RR} 2 \text { and } \mathrm{RO} 14 \text {, resp. }) \text {; } \\
\text { formate: } 2.0 \times \text {, methanol: } 2.4 \times(\mathrm{RR} 2) \text { w or w/o RM, BES } \\
\text { stimulates azo dye reduction with } \mathrm{H}_{2} \text { and formate but has } \\
\text { no effect with acetate and methanol }\end{array}$ & $\begin{array}{l}\text { Dos Santos et al. } \\
(2006)\end{array}$ \\
\hline Acid Orange 6 & AGS at $30{ }^{\circ} \mathrm{C}$ & Acetate & $\mathrm{RF}$ & Max. 0.06 & $\begin{array}{l}\text { Big impact at } \mathrm{RM} / \mathrm{AD}=0.02 \text {; small additional accelerating } \\
\text { effect at } \mathrm{RM} / \mathrm{AD}=0.04 \text { and } 0.06\end{array}$ & $\begin{array}{l}\text { Yemashova and } \\
\text { Kalyuzhnyi } \\
\text { (2006) }\end{array}$ \\
\hline Reactive Red 24 & CAS & YE/Peptone & $\begin{array}{l}\text { Alginate } \\
\text { beads with } \\
\mathrm{AQ}\end{array}$ & NA & $\begin{array}{l}\text { Faster decolorization in comparison to controls with } \\
\text { anthraquinone-free alginate beads }\end{array}$ & $\begin{array}{l}\text { Guo et al. } \\
(2007)\end{array}$ \\
\hline $\begin{array}{l}\text { Reactive Red } 24 \\
\text { Reactive Red } 2 \\
\text { Acid Red } 1 \\
\text { Acid Red } 14 \\
\text { Acid Red } 72 \\
\text { Acid Black } 1\end{array}$ & CAS & YE/Peptone & $\mathrm{BA}$ & $0-4.33$ & $\begin{array}{l}\text { Max. } 1.9 \times, 1.7 \times, 1.5 \times, 1.3 \times, 2.3 \times, 1.8 x \text { (z.o., RR24, RR2, AR1, } \\
\text { AR14, AR72, AB1, respectively); no additional effect at RM/AD > 2.6 }\end{array}$ & $\begin{array}{l}\text { Guo et al. } \\
(2008)\end{array}$ \\
\hline Reactive Red 24 & CAS & YE+Peptone & $\begin{array}{l}\text { Different } \\
\text { AQ- }^{\text {compounds }}\end{array}$ & NA & Increase with all RM tested (not quantified) & $\begin{array}{l}\text { Guo et al. } \\
\text { (2008) }\end{array}$ \\
\hline Acid Orange 7 & Partially digested AAS & Acetate & Graphite & NA & $\begin{array}{l}88 \% \text { decolorization in } 20 \mathrm{~h} \text {, as compared to no } \\
\text { decolorization in } 24 \mathrm{~h} \text { in the absence of graphite }\end{array}$ & $\begin{array}{l}\text { Mezohegyi } \\
\text { et al. (2007) }\end{array}$ \\
\hline
\end{tabular}


Table 4

Impact of redox mediators on the reductive decolorization of azo dyes by mixed microbial cultures in anaerobic continuous reactors

\begin{tabular}{|c|c|c|c|c|c|c|c|}
\hline Azo dye $(A D)^{a}$ & System $^{\mathrm{b}}$ & $\begin{array}{l}\mathrm{HRT}^{\mathrm{c}} \\
(\mathrm{h})\end{array}$ & $\begin{array}{l}\text { Electron } \\
\text { donor }^{\mathrm{d}}\end{array}$ & Mediator $(\mathrm{RM})^{\mathrm{e}}$ & $\begin{array}{l}\mathrm{RM} / \mathrm{AD}- \\
\text { ratio }^{\mathrm{f}}\end{array}$ & Results $^{g}$ & Reference \\
\hline Acid Orange 7 & UASB with AGS & $2-6$ & VFA & AQDS & $\begin{array}{l}\text { Max. } \\
0.105\end{array}$ & $\begin{array}{l}\text { At HRT }=6 \text { h, CRE increases from } 86 \% \text { (w/o RM) to max. 97\%; } \\
\text { effect RM increases with decreasing HRT }\end{array}$ & $\begin{array}{l}\text { Cervantes } \\
\text { et al. }(2001 \mathrm{a}, \mathrm{b})\end{array}$ \\
\hline Reactive Red 2 & UASB with AGS & 5.9 & VFA & AQDS & $\begin{array}{l}\text { Max. } \\
0.97\end{array}$ & $\begin{array}{l}\text { CRE increases with increasing RM conc., from } \sim 40 \% \text { (no RM) } \\
\text { to } 85 \%(R M / A D=0.12) \text { to }>98 \%(R M / A D=0.97)\end{array}$ & $\begin{array}{l}\text { Van der Zee } \\
\text { et al. (2001); }\end{array}$ \\
\hline $\begin{array}{l}\text { Hydrolyzed Reactive } \\
\quad \text { Red } 2\end{array}$ & $\begin{array}{l}\text { EGSB with AGS } \\
\text { at } 55^{\circ} \mathrm{C}\end{array}$ & 10 & $\begin{array}{l}\text { Glucose/ } \\
\text { VFA }\end{array}$ & AQDS & $\begin{array}{l}\text { Max. } \\
0.171\end{array}$ & $\begin{array}{l}\text { Average CRE 95\%, only slightly lower than CRE of RM-free } \\
\text { control reactor ( } 91 \% \text { ); CRE of RM-free reactor more sensitive to } \\
\text { changes in ED/AD-ratio }\end{array}$ & $\begin{array}{l}\text { Dos Santos } \\
\text { et al. (2003) }\end{array}$ \\
\hline $\begin{array}{l}\text { Hydrolyzed Reactive } \\
\text { Red2, textile } \\
\text { wastewater }\end{array}$ & $\begin{array}{l}\text { EGSB with AGS } \\
\text { at different } T\end{array}$ & $\begin{array}{l}10- \\
2.5\end{array}$ & VFA & AQDS & $\begin{array}{l}0.023- \\
0.06\end{array}$ & $\begin{array}{l}\text { Huge impact RM at } 30^{\circ} \mathrm{C} \text {; relatively small effect of AQDS at } 55^{\circ} \text {; } \\
\text { CRE }\left(30^{\circ} \mathrm{C}\right)<\operatorname{CRE}\left(55^{\circ} \mathrm{C}\right) \text { CRE decreases with decreasing HRT }\end{array}$ & $\begin{array}{l}\text { Dos Santos } \\
\text { et al. }(2005 b, c)\end{array}$ \\
\hline Acid Orange 7 & $\begin{array}{l}\text { SBR with AS } \\
\text { (anaerobic } \\
\text { phase) }\end{array}$ & $40^{\mathrm{h}}$ & SA & MQ AQDS & $\begin{array}{l}0.7 \\
0.7 \text { or } \\
1.4\end{array}$ & $\begin{array}{l}\text { CRE increases from } \sim 5 \%(w / o \text { RM) to max. } 25 \% \text { (MQ) and } ~ 70 \% \\
\text { and } ~ 90 \% \text { (AQDS; RM/AD-ratios } 0.7 \text { and } 1.4 \text {, resp.) }\end{array}$ & $\begin{array}{l}\text { Albuquerque } \\
\text { et al. (2005) }\end{array}$ \\
\hline $\begin{array}{l}\text { Hydrolyzed Reactive } \\
\text { Red } 2\end{array}$ & UASB & 5.3 & VFA & PAC & NA & $\begin{array}{l}\text { CRE of PAC-free control reactor } \sim 35 \% \text {, CRE of PAC-amended } \\
\text { reactors } \sim 45 \% \text { and } \sim 95 \% \text { with } 0.4 \text { and } 10 \mathrm{~g} \mathrm{PAC} \mathrm{L} \mathrm{L}^{-1} \text {, respectively }\end{array}$ & $\begin{array}{l}\text { Van der Zee } \\
\text { et al. (2003) }\end{array}$ \\
\hline Acid Orange 7 & $\begin{array}{l}\text { UPBR with } \\
\text { partially } \\
\text { digested AAS }\end{array}$ & $\begin{array}{l}\text { Max. } \\
0.26^{\mathrm{i}}\end{array}$ & Acetate & $\begin{array}{l}\text { GAC (dye-saturated), } \\
\text { Graphite, Aluminium }\end{array}$ & NA & $\begin{array}{l}\text { AC }\left(67 \mathrm{~g} \mathrm{~L}^{-1}\right): 99 \% \text { CRE at } \tau=2 \mathrm{~min} \text {. Graphite }\left(67 \mathrm{~g} \mathrm{~L}^{-1}\right): 77 \% \text { CRE } \\
\text { at } \tau=9.7 \mathrm{~min} \text {. Aluminium }\left(68 \mathrm{~g} \mathrm{~L}^{-1}\right): 33 \% \text { CRE at } \tau=15.8 \mathrm{~min} \text {. }\end{array}$ & $\begin{array}{l}\text { Mezohegyi } \\
\text { et al. (2007) }\end{array}$ \\
\hline
\end{tabular}

a Colour Index generic names; a popular trivial name for Acid Orange 7 is Orange II.

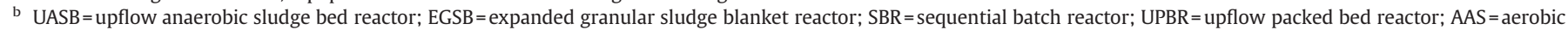
activated sludge (under anaerobic conditions); AGS= anaerobic granular sludge.

c $\mathrm{HRT}=$ hydraulic residence time.

d VFA=volatile fatty acids; $S A=$ hydrolyzed sizing agent (starch derivative).

e $\mathrm{AQDS}=9,10$-Anthraquinone-2,6-disulfonate, $\mathrm{GAC}=$ granular activated carbon, $\mathrm{MQ}=$ Menadione, $\mathrm{PAC}=$ powdered activated carbon .

${ }^{f} \mathrm{RM} / \mathrm{AD}-$ ratio $=$ molar ratio redox mediator: azo dye.

g $\mathrm{AC}=$ activated carbon, $\mathrm{CRE}=$ color removal efficiency; $\mathrm{ED}=$ electron donor; $\tau=$ space time.

h 24 h-cycles with an anaerobic phase of $10.5 \mathrm{~h}$.

i Space time $(\tau)$ rather than hydraulic residence time.

in $\mathrm{mV})$ : AQS(-218)> Lawsone(-139)>AQDS(-184)> Benzyl viologen $(-360)>$ Methyl viologen $(-440)>$, FAD (-219). Moreover, the impact of five quinoid mediators on the reduction of Acid Red 27 by ten pure cultures of distinct classes of bacteria showed large differences between cultures, with different sequences of these five compounds when ranged according to their effect (Rau et al., 2002). These results reflect the diverging and apparently $E_{0}^{\prime}$-independent preferences of biological mediator reduction. In contrast, in chemical systems, the mediator's $E_{0}^{\prime}$ may very well be more important in determining its reduction rate. However, none of the studies listed in Table 5 allows for either confirming or rejecting such a relationship.

From the above it is clear that mediated azo dye reduction, at least in biological systems, is largely independent of the mediator's $E_{0}^{\prime}$, and that this independence is probably mostly due to reduction of the mediator rather than to that of the dye. Therefore, it is likely that the mechanism of mediator reduction is responsible for the observed different impacts of different mediators. However, since diverging mediator preferences of different microorganisms suggest they use distinct enzyme systems to reduce mediators, any possible correlation between the mediators' physico-chemical properties (e.g. size, charge, functional groups, etc.) and their impact on azo dye reduction is inevitably vague. The clearest trend, one that seems almost general, is that AQS is a more powerful mediator than AQDS (Rau et al., 2002; Dos Santos et al., 2004a; Hong et al., 2007). Although it cannot be excluded that this difference is solely a result of $E_{0}^{\prime}$ differences, it is possible that the presence of an additional sulfonate group in AQDS diminishes the ease at which the compound approaches the enzyme.

\subsubsection{Ability to cross the cell membrane}

A property of electron shuttling compounds that deserves attention is the ability to cross cell membranes. It has been demonstrated that the azoreductase activity of cell extracts can be much higher than that of intact cells and that the cell membrane forms a barrier for dyes and mediators (Kudlich et al., 1997; Russ et al., 2000). Hence, mediated azo dye reduction by Sphingomonas xenophaga, with the sulfonated quinone AQS as the optimum mediator, is presumably associated with the membrane-bound respiratory chain enzyme NADH:ubiquinone oxidoreductase (Kudlich et al., 1997; Rau et al., 2002), and several other species are likely to use

\footnotetext{
Notes to Table 3:

a Colour Index generic names are given whenever available; popular trivial names are Naphthol Blue Black (Acid Black 1), Methyl Orange (Acid Orange 52), Amaranth (Acid Red 27), Sunset Yellow (Food Yellow 3).

b AAS=aerobic activated sludge (under anaerobic conditions), AGS= anaerobic granular sludge; CAS= mixture of salt-tolerant bacteria.

c $\mathrm{VFA}=$ volatile fatty acids (in these cases mixtures of acetate, propionate and butyrate), $Y E=$ yeast extract.

d $\mathrm{AD}$-metab. = metabolites of anaerobic reduction of the azo dyes Direct Blue 53 or Direct Blue 71 (autocatalysis); $\mathrm{AQ}=9,10-\mathrm{Anthraquinone;} \mathrm{AQDS=9,10-Anthraquinone-2,6-disulfonate;}$

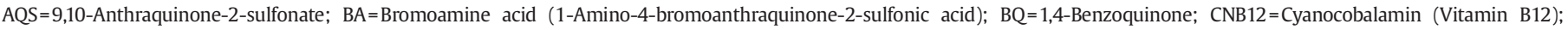
LAW=Lawsone (2-Hydroxy-1,4-naphthoquinone); RF=Riboflavin.

e RM/AD-ratio = molar ratio redox mediator: azo dye (unless otherwise stated).

$f$ ' $x$ ' expresses the $x$-fold increase of the pseudo first-order reduction rate constants (not marked) or zero-order reduction rate constants (marked with 'z.o') as compared to the RMfree controls; avg. = average; resp. = respectively; dye names are logically abbreviated.

g Henna leaves are a source of Lawsone.

h assuming 2 mol catalytic aromatic amine per mol reduced azo dye.

i 1-Aminoanthraquinone; 1-Amino-2-bromo-4-hydroxyanthraquinone; 1,4-Diaminoanthraquinone; 2-Aminoanthraquinone; 1,4-Dihydroxyanthraquinone.
} 
Table 5

Impact of redox mediators on the reductive decolorization of azo dyes by different electron donors under abiotic conditions

\begin{tabular}{|c|c|c|c|c|c|}
\hline Azo dye ${ }^{a}$ & Electron donor ${ }^{\mathrm{b}}$ & Mediator $^{\mathrm{c}}$ & RM/AD-ratio ${ }^{d}$ & Results $^{\mathrm{e}}$ & Reference \\
\hline Acid Red 27 & $\begin{array}{l}\text { NADPH } \\
\text { NADPH }\end{array}$ & FAD & 3.1 & Without FAD: less than $5 \%$ reduction & $\begin{array}{l}\text { Russ et al. } \\
(2000)\end{array}$ \\
\hline $\begin{array}{l}\text { Acid Red } 27 \text { (Acid Orange 7) } \\
\text { (Acid Orange 52) (Acid Yellow 23) } \\
\text { (4-AB) (4-HAB) }\end{array}$ & $\begin{array}{l}\text { NADPH } \\
\text { (generating system) }\end{array}$ & BV & 13.3 & $\begin{array}{l}\text { Acid Red 27: increase (not quantified); } \\
\text { other azo compounds: no reaction }\end{array}$ & $\begin{array}{l}\text { Semdé et al. } \\
\text { (1998) }\end{array}$ \\
\hline Reactive Orange $140.15 \mathrm{mM}$ & Sulfide & $\mathrm{RF}$ & 0.13 & $31-45 \times$ & $\begin{array}{l}\text { Cervantes } \\
\text { et al. (2006) }\end{array}$ \\
\hline Acid Orange $70.25-0.3 \mathrm{mM}$ & Sulfide & AN AQDS & $\begin{array}{l}\text { Max. } 4 \\
\text { Max. } 2\end{array}$ & $\begin{array}{l}\text { Max. } 13 \times \\
\text { Max. } 105 \times\end{array}$ & $\begin{array}{l}\text { Van der Zee } \\
\text { et al. (2000) }\end{array}$ \\
\hline Acid Orange $70.25-0.3 \mathrm{mM}$ & Sulfide & Autoclaved AGS & $\begin{array}{l}\text { Max } 36 \text { gVSS } \\
\mathrm{mmol}^{-1}\end{array}$ & Max. $10 \times$ & $\begin{array}{l}\text { Van der Zee } \\
\text { et al. (2000) }\end{array}$ \\
\hline Reactive Red $20.3 \mathrm{mM}$ & $\begin{array}{l}\text { Sulfide at different } \\
T\end{array}$ & AQS & 0.04 & $\begin{array}{l}\text { 1.2-fold decrease of activation energy } \\
\text { due to AQS }\end{array}$ & $\begin{array}{l}\text { Dos Santos } \\
\text { et al. } \\
(2004 a)\end{array}$ \\
\hline Hydrolyzed Reactive Red $20.3 \mathrm{mM}$ & Sulfide $55{ }^{\circ} \mathrm{C}$ & AQS & 0.04 & $1.3-1.5 \times$ & $\begin{array}{l}\text { Dos Santos } \\
\text { et al. } \\
(2004 a)\end{array}$ \\
\hline $\begin{array}{l}\text { 4-AAB bound to epoxy-activated } \\
\text { Sepapharose beads }\end{array}$ & ZVI & JUG HEM, Chlorophyll, HA & $\begin{array}{l}10100 \mathrm{~g} \mathrm{mmol}^{-1} \\
2.5 \mathrm{gC} \mathrm{mmol}^{-1}\end{array}$ & $\begin{array}{l}\text { Without RM: no dye reduction; JUG: complete } \\
\text { reduction f; other RM: some reduction }\end{array}$ & Weber \\
\hline $\begin{array}{l}\text { 4-AAB bound to epoxy-activated } \\
\text { Sepapharose beads }\end{array}$ & ZVI & $\begin{array}{l}\text { Pond sediment; pond sediment- } \\
\text { associated water; (water-free } \\
\text { sediment) }\end{array}$ & NA & $\begin{array}{l}\text { Pond sediment: very slow but steady reduction; } \\
\text { pond sediment-associated water: some initial } \\
\text { reduction after which the reaction stops; } \\
\text { water-free sediment: no reduction }\end{array}$ & $\begin{array}{l}\text { Weber } \\
(1996)\end{array}$ \\
\hline $\begin{array}{l}\text { Pond sediment-adsorbed Disperse } \\
\text { Blue } 79\end{array}$ & ZVI & Pond sediment $\mathrm{w}$ or $\mathrm{w} / \mathrm{o}$ JUG & NA & Some reduction; small effect of JUG & $\begin{array}{l}\text { Weber } \\
(1996)\end{array}$ \\
\hline Acid Yellow 9 & Cathodic reduction & AQS & Max. 0.15 & Without RM: no dye reduction & $\begin{array}{l}\text { Bechtold } \\
\text { et al. (1997) }\end{array}$ \\
\hline
\end{tabular}

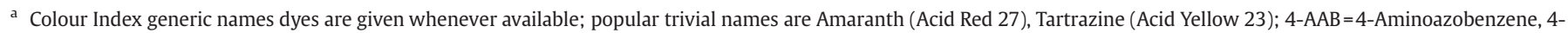
$\mathrm{HAB}=4$-Hydroxyazobenzene.

b ZVI=Zero-valent iron.

c AGS = anaerobic granular sludge, $A N=1$-Amino-2-naphthol, AQ=9,10-Anthraquinone, AQDS=9,10-Anthraquinone-2,6-disulfonate, 9,10-AQS=Anthraquinone-2-sulfonate, BV=Benzyl

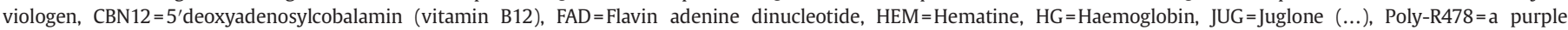
polyanthraquinone dye, $\mathrm{RA}=$ Resazurin, $\mathrm{RF}=$ Riboflavin.

d RM/AD-ratio= molar ratio redox mediator: azo dye (unless otherwise stated).

e ' $x$ ' expresses the $x$-fold increase of the pseudo first-order reduction rate constants (not marked) or zero-order reduction rate constants (marked with 'z.o') as compared to the RMfree controls; avg. = average; resp. $=$ respectively.

f Aniline recovery $80 \%$, equal to the aniline recovery with free 4-AAB.

similar membrane-associated enzyme systems. However, as has recently been shown for E. coli, the mechanism can also be based on intracellular enzymes in combination with a mediator that diffuses through the cell membrane. E. coli's mediator-dependent 'azoreductase' was identified as two cytosolic oxygen-insensitive nitroreductases, acting as quinone reductases. The mediator was Lawsone, the only quinone with a mediator-feasible $E_{0}^{\prime}$ that could be reduced by the enzymes (Rau and Stolz, 2003). Lawsone's apparent ability to cross the cell membrane is probably related to its small size and its relative lipophilicity (no charged substituents). Depending on the preferences of intracellular reductases in other species, there may be more shuttling compounds with membranecrossing properties that could act as powerful mediators for azo dye reduction.

\subsubsection{Solubility vs. non-solubility}

While the large majority of the studies listed in Tables 1-5 dealt with water-soluble compounds as redox mediators for azo dye reduction, a few investigations demonstrated redox-mediating properties of non-soluble materials. Especially activated carbon (Van der Zee et al., 2003; Mezohegyi et al., 2007), to a lesser extent graphite (Mezohegyi et al., 2007) and to some extent alginate beads with immobilized anthraquinone (Guo et al., 2007) have been shown to accelerate azo dye reduction in biological systems. As graphite, which was shown to act as mediator in contrast to the nonconductive material aluminium (Mezohegyi et al., 2007), does not contain specific groups on its surface, it is clear that catalysis of biological azo dye reduction does not necessarily depend on redoxactive surface groups. Nevertheless, the considerably higher dye reduction kinetics obtained with activated carbon as compared to graphite (Mezohegyi et al., 2007) indicates that surface groups play an important role. Therefore, activated carbon or other insoluble materials with surface-associated or entrapped redox-active functional groups can be considered the most logical candidates as insoluble redox mediators. The catalytic properties of such insoluble materials enable their use as biologically regenerable RM in anaerobic bioreactors. As these materials can be retained in the reactor for prolonged time, they are attractive alternatives to soluble RM that need to be dosed continuously.

\subsection{Mediator/dye-ratio}

Tables 1-5 show that a wide range of molar mediator/dye-ratios has been applied. In several studies, two or more ratios have investigated, usually by changing the mediator concentration (Roxon et al., 1967; Walker et al., 1971; Kudlich et al., 1997; Van der Zee et al., 2000; Cervantes et al., 2001b; Van der Zee et al., 2001; Rau et al., 2002; Dos Santos et al., 2003; Field and Brady, 2003; Dos Santos et al., 2004b; Albuquerque et al., 2005; Dos Santos et al., 2005b; Dos Santos et al., 2005c; Dos Santos et al., 2005d; Yemashova and Kalyuzhnyi, 2006; Hong et al., 2007). Dye reduction rates increase with increasing mediator concentrations. Mediator gradients typically show that the effect is especially strong at low mediator/dye ratios and levels off at higher ratios. The mediator/dye-ratios beyond which there is no additional effect of raising the mediator concentration are mostly rather high: it is not unusual that maximum azo dye reduction rates have not yet been reached at molar ratios as high as 1 (Kudlich et al., 1997; Van der Zee et al., 2001; Dos Santos et al., 2004b). 


\subsection{Temperature}

Reaction rates will increase with increasing temperatures because a higher fraction of the molecules will have a kinetic energy higher than the activation energy. This effect, as described by the Arrhenius equation, will be more pronounced if the reaction's activation energy is higher. Raising the temperature will thus result in a relatively higher increase of the rate of non-mediated azo dye reduction (high activation energy) in comparison to that of mediated azo dye reduction (lower activation energy). It can be expected that chemical azo dye reducing systems behave accordingly, as appears to be confirmed by the results of Dos Santos et al. (2004a), the only study in Table 5 that investigated chemical azo dye reduction at different temperatures. In this study it was observed that the first-order reaction rate constant of non-mediated reduction of Reactive Red 2 $(0.3 \mathrm{mM})$ by sulfide $(4.5 \mathrm{mM})$ was 2.2 -fold higher at $55{ }^{\circ} \mathrm{C}$ than at $30^{\circ} \mathrm{C}$, and AQS $(12 \mu \mathrm{M})$ increased these rate constants 1.9 -fold and 1.7fold at $30{ }^{\circ} \mathrm{C}$ and $55{ }^{\circ} \mathrm{C}$, respectively (Dos Santos et al., 2004a). In biological systems, there will be limits to the temperature range of individual cultures and enzyme systems, as has been demonstrated for non-mediated azo dye reduction by several pure cultures (Chang and Kuo, 2000; Chang et al., 2001; Yu et al., 2001; Pearce et al., 2006; Angelova et al., 2008) but not for mediated azo dye reduction. Mixed cultures such as sludge will undergo population shifts when the temperature is changed. It must be taken into account, therefore, that the results of the studies by Dos Santos et al. (2005c) on azo dye reduction by mesophilic and thermophilic sludge may at least partly reflect changes of biomass properties.

Most of the results from these studies show the Arrhenius trends, i.e. higher basic azo dye reduction rates and a relatively smaller impact of mediators with thermophilic sludge as compared to with mesophilic sludge. For example, the first-order reaction rate constant of non-mediated reduction of hydrolyzed Reactive Red $2(0.3 \mathrm{mM})$ was 5.6 -fold higher with thermophilic sludge $\left(55^{\circ} \mathrm{C}\right)$ than with mesophilic sludge $\left(30^{\circ} \mathrm{C}\right)$, and AQS $(12 \mu \mathrm{M})$ increased these rate constants 3.8fold and 2.3 -fold at $30{ }^{\circ} \mathrm{C}$ and $55{ }^{\circ} \mathrm{C}$, respectively (Dos Santos et al., 2005c). Comparison of these results with the ones cited above for the reaction with sulfide, shows a general feature of sludge vs. sulfide, i.e. a much stronger effect of temperature on non-mediated azo dye reduction by sludge than by sulfide. This phenomenon may be the result of differences in the microbial composition between mesophilic and thermophilic sludge. Alternatively, it may also reflect a temperature-dependent change of the activation energy, similar to that observed for non-mediated azo dye reduction by two distinct pure cultures (Angelova et al., 2008). In contrast, explanations based on the release of intracellular mediators as a result of higher cell decay at higher temperatures are probably not valid: it would imply that, due to the higher pool of mediators, the impact of additional mediators is lower with sludge than with sulfide, something which is obviously not the case. Some of the results from the studies by Dos Santos and coworkers are not in agreement with the Arrhenius trends. The anomalous decrease of the rate of both mediated and non-mediated azo dye reduction observed at temperatures beyond the optimum temperature of $60{ }^{\circ} \mathrm{C}$ (Dos Santos et al., 2003) probably reflects the temperature optimum of the sludge inoculum. In contrast, there is no clear explanation for the lower relative impact of AQDS on the reduction of hydrolyzed Reactive Red 2 at $45{ }^{\circ} \mathrm{C}$ than at 55 and $60{ }^{\circ} \mathrm{C}$ (Dos Santos et al., 2003), and for the higher relative impact of riboflavin on the reduction of Reactive Orange 14 at $55^{\circ} \mathrm{C}$ than at $30^{\circ} \mathrm{C}$ (Dos Santos et al., 2005b).

\subsection{Electron donors}

RM have been shown to accelerate or even enable azo dye reduction by microorganisms from many distinct taxonomic and trophic groups. The primary electron donor of biological azo dye reduction may therefore play a role in determining azo dye reduction rates: in individual cultures by changing enzyme activities, and in mixed cultures by stimulating specific (trophic groups of) microorganisms. The substrate type may thus affect both the rates of direct (non-mediated) azo dye reduction and those of mediator reduction, albeit not necessarily to the same extent.

Four of the studies listed in Table 4, all of them using anaerobic granular sludge, compared the effects of different substrates on mediated and non-mediated azo dye reduction (Van der Zee et al., 2001; Dos Santos et al., 2003; Dos Santos et al., 2005b; Dos Santos et al., 2006). In one of these studies, evidence was provided for a substrateselective effect on the reduction of a mediator: It was observed that acetate stimulated the AQDS-mediated reduction of Reactive Red 2 by AQDS-adapted sludge much more than propionate, butyrate or hydrogen. In contrast, acetate neither stimulated non-mediated dye reduction, nor did it have a pronounced effect on mediated azo dye reduction by non-adapted adapted sludge. Therefore, these results suggest that enrichment of bacteria or enzyme systems that coupled AQDS-reduction to oxidation of acetate had taken place (Van der Zee et al., 2001). The other substrate effect studies were all performed with consortia not previously adapted to the mediator. Azo dye reduction rates were different with each particular substrate, and mediators always increased these rates, albeit to different extents. The values mentioned in the 'Results' column in Table 3 express, for each particular substrate investigated, the impact of the mediator (riboflavin or AQDS) on the rate of azo dye reduction, and should therefore mostly be interpreted as the effect of this substrate on mediator reduction. In some cases, a positive correlation between these values and the basic non-mediated rates has been observed. For example, both the non-mediated rates of azo dye reduction by thermophilic sludge and the relative impact of AQDS increased along the substrate range volatile fatty acids (VFA)Glucose+VFA-Glucose- $\mathrm{H}_{2}$ (Dos Santos et al., 2003), and a similar correlation was found for azo dye reduction by thermophilic sludge and the relative impact of riboflavin with Endogenous substrate-AcetateFormate- $\mathrm{H}_{2}$ (Dos Santos et al., 2006). In other cases, however, the observed correlation was negative. For example, along the substrate range acetate-methanol-formate, the non-mediated azo dye reduction rates by mesophilic sludge increased but the relative impact of riboflavin decreased (Dos Santos et al., 2006). In addition, contradicting results have been reported for the effect of hydrogen on mediated vs. nonmediated Reactive Red 2 reduction by unadapted mesophilic sludge: whereas both studies reported that hydrogen was the substrate with the highest dye reduction rates, the high relative impact of riboflavin observed by Dos Santos et al. (2005a) contrasts sharply to the rather small relative impact of AQDS observed by Van der Zee et al. (2001). From these conflicting trends it is impossible to draw conclusions with respect to substrate preferences for mediator reduction. It is obvious, however, that the overall azo dye reduction process (as well as nonmediated azo dye reduction) is much more associated with hydrogen and glucose oxidation than with the oxidation of VFA.

\subsection{Trophic groups and selective inhibitors}

The higher azo dye reduction rates with glucose as compared to with VFA (as discussed in the previous sub-section) suggest that fermentative bacteria contribute more to azo dye reduction than acetogens and methanogens. More information about the involvement of fermentative and methanogenic bacteria was obtained in experiments to the effect of selective inhibitors on azo dye reduction by anaerobic granular sludge in the presence of different electron donors (Dos Santos et al., 2005b; Dos Santos et al., 2006). Vancomycin, a selective inhibitor of Gram-positive bacteria, was shown to suppress both non-mediated and mediated azo dye reduction: it decreased the reduction rates of Reactive Red 2 and Reactive Orange 14 by both mesophilic and thermophilic anaerobic sludge with $50-90 \%$ and $30-55 \%$, with glucose and methanol as the electron donors, respectively, regardless of the presence or absence of riboflavin. As 
most fermentatives are Gram-positive bacteria, these observations suggest that their involvement in azo dye reduction is considerable.

In contrast to vancomycin, 2-Bromoethanesulfonate (BES), the selective inhibitor of methanogens, usually did not have much effect on azo dye reduction by granular sludge. The only exception was a considerable increase of the non-mediated reduction rates of Reactive Red 2 and Reactive Orange 14 by thermophilic sludge with hydrogen as the electron donor. In addition, BES also stimulated, albeit weakly, the non-mediated reduction of Reactive Red 2 in some other situations, i.e. with thermophilic sludge and glucose or formate, as well with mesophilic sludge and hydrogen. These observations point at substrate competition between methanogenesis and dye reduction, indicating that non-mediated azo dye reduction was to some extent linked to oxidation of hydrogen and formate (directly), or to fermentation of glucose (indirectly, by production of methanogenic substrates). Interestingly, BES did not raise any of the azo dye reduction rates in the presence of the mediator riboflavin. On the contrary, its effect, although insignificant, was always negative (Dos Santos et al., 2005b; Dos Santos et al., 2006). This suggestion of a small contribution of methanogens to riboflavin reduction was confirmed by the results of pure culture assays with hydrogenotrophic methanogens. All of the three strains used in these assays, the thermophiles Methanothermobacter thermoautotrophicus $\Delta \mathrm{H}$ and a Methanothermobacter-related strain NJ1 and the mesophile Methanosarcina barkeri, were found capable of riboflavin-mediated azo dye reduction. In contrast, only the mesophilic strain could reduce the azo dye Reactive Red 2 in the absence of riboflavin (Dos Santos et al., 2006). The combined results of these sludge and pure culture studies indicate that the contribution of methanogens to azo dye reduction by anaerobic sludge is ambiguous but generally limited.

\section{Role of redox mediators on reductive dechlorinations}

\subsection{Introduction}

Polychlorinated compounds (PCC) constitute an important group of contaminants widely spread in wastewaters, groundwater, soils and off-gasses, due to improper handling, leakage, etc. Due to their potential hazard for the environment and public health, many PCC are listed as priority pollutants by the USA environmental protection agency (EPA). PCC are commonly used as degreasing agents and solvents in the metal-industry, as cooling fluids in refrigerators, as pesticides, fungicides or insecticides, or as dielectric fluids or flame retardants (Van Eekert, 1999).

The role of RM, with different structures and properties, on the reductive dechlorination of different PCC has been tested during the last two decades. Tables 6 and 7 summarize the impacts of these RM on reductive dechlorination processes. The impact of RM on dechlorinating reactions varies among the different reaction systems evaluated. Some RM increase the rate and extent of dechlorination of distinct PCC by several orders of magnitude, under different experimental conditions. However, several dechlorinating reactions were hardly affected or were even inhibited when different RM were incorporated. A number of variables are at stake determining the impact of RM in different dechlorinating reactions, which will be discussed in the following sections.

\subsection{Main factors determining the input of RM in reductive dechlorinations}

\subsection{1. $p H$}

A few studies report on the effect of $\mathrm{pH}$ on reductive dechlorination processes in the presence of different RM. For instance, abiotic incubations for the reductive dechlorination of hexachloroethane (HCA) by sulfide $(0.25 \mathrm{mM})$, in the presence of soil humic acids (HA, $25 \mathrm{mg} \mathrm{C/L}$ ), showed a direct relationship between the pseudo-firstorder rate constant $(k)$ for the reduction of HCA and the $\mathrm{pH}$ (Curtis and Reinhard, 1994). The $k$ value increased up to $\sim 5$-fold by varying the $\mathrm{pH}$ from 7.2 to 8.0 in the assays. The first-order dependence of the reaction on $\mathrm{pH}$ was attributed to deprotonation of the redox active groups in HA (e.g. quinones), which was previously proposed during the reduction of methyl parathion by reduced quinones at different $\mathrm{pH}$ values (Tratnyek and Macalady, 1989, see below).

The last hypothesis was corroborated by further experiments indicating that the $k$ value for HCA reduction by anthrahydroquinone2,6-disulfonate $\left(\mathrm{AH}_{2} \mathrm{QDS}\right)$ strongly depends on the $\mathrm{pH}$. Curtis and Reinhard (1994) postulated that the overall rate expression for HCA reduction was considered to be the sum of three independent, parallel, first-order reactions:

$k^{\prime}{ }_{\mathrm{HCA}}=k_{0}\left[\mathrm{~A}(\mathrm{OH})_{2}\right]+k_{1}\left[\mathrm{~A}(\mathrm{OH}) \mathrm{O}^{-}\right]+k_{2}\left[\mathrm{~A}(\mathrm{O})_{2}^{2-}\right]$

Where $\left[\mathrm{A}(\mathrm{OH})_{2}\right],\left[\mathrm{A}(\mathrm{OH}) \mathrm{O}^{-}\right]$, and $\left[\mathrm{A}(\mathrm{O})_{2}^{2-}\right]$ represent the concentrations of the fully protonated, the monophenolate, and the diphenolate forms of $\mathrm{AH}_{2} \mathrm{QDS}$ and $k_{0}, k_{1}$, and $k_{2}$ are the corresponding rate constants. It was observed that $k_{2}$ was $\sim 8$-fold higher than $k_{1}$ while $k_{0}$ was not different from zero, indicating that the dechlorination process mediated by quinones proceeds faster at high $\mathrm{pH}$ levels.

Similar results occurred during the reductive dechlorination of HCA and carbon tetrachloride (CT) by cysteine $(5 \mathrm{mM})$ in the presence of iron porphyrin $(30 \mu \mathrm{M})$ at different $\mathrm{pH}$ values. The second-order rate constants for the reductive dechlorination of HCA and CT increased up to 4- and 5-fold, respectively, by raising the $\mathrm{pH}$ from 6.0 to 9.3 (Perlinger et al., 1998).

Recent experiments suggest that different reversible functional groups are involved in redox reactions catalyzed by humic substances depending on the $\mathrm{pH}$ prevailing on the reaction system. Evidence was provided that non-quinoid (NQ) structures in humus have electroncarrying capacity (ECC) at $\mathrm{pH} 6.5$; whereas different quinoid $\left(\mathrm{Q}_{1}\right.$ and $\mathrm{Q}_{2}$ ) structures showed ECC at pH 8 (Ratasuk and Nanny, 2007). $\mathrm{Q}_{1}$ was defined as quinone moieties with electron-withdrawing groups adjacent to $Q_{1}$, while $Q_{2}$ contains either no substituents near the quinone or have nearby electron-donating groups with additional substituents hindering hydrogenolysis through steric interactions. NQ sites accounted for $21-56 \%$ of ECC in different humic materials; whereas $\mathrm{Q}_{1}$ and $\mathrm{Q}_{2}$ accounted for $13-58 \%$ and $8-50 \%$ of the total ECC, respectively (Ratasuk and Nanny, 2007).

\subsubsection{Temperature}

As described above (azo dyes section), reaction rates will enhance with increasing temperatures because a higher fraction of the molecules will have a kinetic energy higher than the activation energy. This was evident during the reductive dechlorination of HCA by sulfide $(1 \mathrm{mM})$, in the presence and in the absence of juglone, at different incubation temperatures (Perlinger et al., 1996). The reduction rate of HCA by sulfide increased $\sim 15$-fold by raising the temperature from $25^{\circ} \mathrm{C}$ to $65^{\circ} \mathrm{C}$ in nonmediated reactions; whereas the rate of juglone-mediated reduction increased $\sim 10$-fold. The inferior effect of temperature on the reduction rate of HCA observed in juglone-mediated systems, compared to controls lacking juglone, could be attributed to differences in the apparent activation energy (Ea) required in the reactions. In agreement with this observation, a decrease in the Ea was determined when dechlorinating reactions were conducted with different concentrations of juglone. Certainly, the Ea obtained in non-mediated reactions was $75 \pm 5 \mathrm{~kJ} / \mathrm{mol}$; whereas in the presence of $20 \mu \mathrm{M}$ juglone, Ea decreased to $52 \pm 5 \mathrm{~kJ} / \mathrm{mol}$, and in the presence of $200 \mu \mathrm{M}$ juglone, Ea was $46 \pm 7 \mathrm{~kJ} / \mathrm{mol}$ (Perlinger et al., 1996). A lower Ea in mediated reactions implies that the presence of a RM facilitate the reactants to achieve their reactive state, thus accelerating the transfer of electrons.

Comparison of the reduction rates of $\mathrm{HCA}$ by $\mathrm{AH}_{2} \mathrm{QDS}$, achieved at $25^{\circ} \mathrm{C}$ (Kappler and Haderlein, 2003) and at $50{ }^{\circ} \mathrm{C}$ (Curtis and Reinhard, 1994), in both cases conducted at $\mathrm{pH} 8.2$ and a $\mathrm{AH}_{2} \mathrm{QDS} / \mathrm{HCA}$-ratio of 200, also agrees with the Arrhenius equation. Indeed, the $k$ value 
Table 6

Impact of redox mediators on reductive dechlorination reactions under abiotic conditions

\begin{tabular}{|c|c|c|c|c|c|}
\hline Reaction & $\begin{array}{l}\text { Electron } \\
\text { donor/system }\end{array}$ & Mediator & Ratio pollutant/mediator & Results & References \\
\hline $\mathrm{C}_{2} \mathrm{Cl}_{6} \rightarrow \mathrm{C}_{2} \mathrm{Cl}_{4}$ & $\begin{array}{l}\text { Sulphide or Fe } \\
\text { (II) }\end{array}$ & $\begin{array}{l}\text { HEM, BOM } \\
\text { or HA }\end{array}$ & $\begin{array}{l}\text { Molar ratio } \mathrm{C}_{2} \mathrm{Cl}_{6} / \mathrm{HEM} \text { of } 0.025 ; 0.1 \mu \mathrm{mol} \\
\mathrm{C}_{2} \mathrm{Cl}_{6} / \mathrm{mg} \mathrm{C}-\mathrm{HA} ; 0.125 \mu \mathrm{mol} \mathrm{C}_{2} \mathrm{Cl}_{6} / \mathrm{mg} \mathrm{BOM}\end{array}$ & $\begin{array}{l}\text { 7- to } 45 \text {-fold higher first-order reduction rates in } \mathrm{RM} \text { amended } \\
\text { incubations compared to controls }\end{array}$ & $\begin{array}{l}\text { Curtis and } \\
\text { Reinhard } \\
\text { (1994) }\end{array}$ \\
\hline $\mathrm{CCl}_{4} \rightarrow \mathrm{CHCl}_{3}$ & $\begin{array}{l}\text { Sulphide or Fe } \\
\text { (II) }\end{array}$ & HEM or HA & $\begin{array}{l}\text { Molar ratio } \mathrm{CCl}_{4} / \mathrm{HEM} \text { of } 0.025 ; 0.1 \mu \mathrm{mol} \\
\mathrm{CCl}_{4} / \mathrm{mg} \mathrm{C}-\mathrm{HA}\end{array}$ & $\begin{array}{l}\text { 2- to } 7 \text {-fold and 6- to } 18 \text {-fold higher first-order reduction rates } \\
\text { in RM amended incubations compared to controls in Fe(II) and } \\
\text { sulphide supplemented incubations, respectively }\end{array}$ & $\begin{array}{l}\text { Curtis and } \\
\text { Reinhard } \\
(1994)\end{array}$ \\
\hline $\mathrm{CHBr}_{3} \rightarrow \mathrm{CH}_{2} \mathrm{Br}_{2}$ & $\begin{array}{l}\text { Sulphide or Fe } \\
\text { (II) }\end{array}$ & HEM or HA & $\begin{array}{l}\text { Molar ratio } \mathrm{CHBr}_{3} / \mathrm{HEM} \text { of } 0.025 ; 0.1 \mu \mathrm{mol} \\
\mathrm{CHBr}_{3} / \mathrm{mg} \mathrm{C}-\mathrm{HA}\end{array}$ & $\begin{array}{l}\text { No significant impact of RM with } \mathrm{Fe}(\mathrm{II}) \text { as electron donor. } \\
\text { Hematin stimulated the reduction of } \mathrm{CHBr}_{3} \text { by sulphide, which } \\
\text { was not reduced in the absence of RM }\end{array}$ & $\begin{array}{l}\text { Curtis and } \\
\text { Reinhard } \\
(1994)\end{array}$ \\
\hline $\mathrm{C}_{2} \mathrm{Cl}_{6} \rightarrow \mathrm{C}_{2} \mathrm{Cl}_{4}$ & Sulphide & JUG & Molar ratio of $4 \times 10^{-3}$ & $\begin{array}{l}\text { 10-fold higher first-order reduction rates in JUG-amended } \\
\text { incubations compared to controls }\end{array}$ & $\begin{array}{l}\text { Perlinger } \\
\text { et al. (1996) }\end{array}$ \\
\hline $\begin{array}{l}\mathrm{TCE} \rightarrow \mathrm{VC}, 1,1-\mathrm{DCE}+ \\
\text { 1,2-DCE, } \\
\text { acetylene, } \\
\text { ethylene, } \\
\text { ethane }\end{array}$ & ZVI & NOM & 0.625 to $1.25 \mu \mathrm{mol} \mathrm{TCE} / \mathrm{mg}$ NOM & $\begin{array}{l}\text { Decrease by } 21 \text { to } 39 \% \text { on the first-order reduction rates by } \\
\text { increasing the amount of NOM }\end{array}$ & $\begin{array}{l}\text { Tratnyek } \\
\text { et al. (2001) }\end{array}$ \\
\hline $\mathrm{CCl}_{4} \rightarrow \mathrm{UP}$ & ZVI & $\begin{array}{l}\text { AQDS, JUG, } \\
\text { FA or HA }\end{array}$ & $\begin{array}{l}\text { Molar ratio of } 0.021 \text { to } 2.1 \text { and } 0.017 \text { to } 1.7 \text { for } \\
\text { AQDS and JUG, respectively; } 4.25 \text { to } 17 \mu \mathrm{mol} \\
\mathrm{CCl}_{4} / \mathrm{mg} \mathrm{FA} \text { and } 17 \mu \mathrm{mol} \mathrm{CCl} / \mathrm{mg} \mathrm{HA}\end{array}$ & $\begin{array}{l}\text { 2- and 3-fold higher first-order reduction rates in JUG and AQDS } \\
\text { amended incubations, respectively, compared to controls. } \\
\text { Decrease by } 35 \% \text { and } 50 \% \text { on the first-order reduction rates by } \\
\text { increasing the amount of FA and HA, respectively. }\end{array}$ & $\begin{array}{l}\text { Tratnyek } \\
\text { et al. (2001) }\end{array}$ \\
\hline $\begin{array}{c}\mathrm{C}_{2} \mathrm{Cl}_{4} \rightarrow \text { ethylene } \\
\text { and ethane }\end{array}$ & $\begin{array}{l}\text { Titanium (III) } \\
\text { citrate }\end{array}$ & $\begin{array}{l}\text { Solubilized } \\
\text { MPP (with } \\
\text { cosolvent) }\end{array}$ & Molar ratio of 30 & $\begin{array}{l}\text { Complete } \mathrm{C}_{2} \mathrm{Cl}_{4} \text { conversion in } 24 \mathrm{~h} \text { for RM-amended cultures. } \\
\text { No conversion of } \mathrm{C}_{2} \mathrm{Cl}_{4} \text { in the absence of RM }\end{array}$ & $\begin{array}{l}\text { Dror and } \\
\text { Schlautman } \\
\text { (2004) }\end{array}$ \\
\hline $\begin{array}{c}\mathrm{C}_{2} \mathrm{Cl}_{4} \rightarrow \text { ethylene } \\
\text { and ethane }\end{array}$ & $\mathrm{Pd} / \mathrm{ZVI}^{\mathrm{a}}$ & $\begin{array}{l}\text { HA }{ }^{\mathrm{b}}, \mathrm{AQDS}, \\
\mathrm{LAW} \text { or HQ }\end{array}$ & $\begin{array}{l}0.075 \text { to } 0.2 \mu \mathrm{mol} \mathrm{C}_{2} \mathrm{Cl}_{4} / \mathrm{mg} \mathrm{HA} \text {. For the model } \\
\text { RM the molar ratio was } 0.012\end{array}$ & $\begin{array}{l}\text { 2- to 3-fold higher first-order reduction rates in model RM } \\
\text { amended incubations compared to controls. HA decreased } \\
\text { dechlorination rates compared to controls }\end{array}$ & $\begin{array}{l}\text { Doong and } \\
\text { Lai (2005) }\end{array}$ \\
\hline $\mathrm{CCl}_{4} \rightarrow \mathrm{CHCl}_{3}+\mathrm{UP}$ & $\begin{array}{l}\text { Sulphide or } \\
\text { cysteine }\end{array}$ & $\begin{array}{l}\text { AQDS, BQ, } \\
\text { JUG, NQ, } \\
\text { LAW or MQ }\end{array}$ & Molar ratio of 0.02 & $\begin{array}{l}\text { 4- to 5-fold higher first-order reduction rates in LAW and AQDS } \\
\text { amended incubations, compared to controls. For the rest of the } \\
\text { RM the enhancement on the first-order reduction rates varied } \\
\text { from } 20 \text { - up to } 71 \text {-fold }\end{array}$ & $\begin{array}{l}\text { Doong and } \\
\text { Chiang } \\
(2005)\end{array}$ \\
\hline $\begin{aligned} \mathrm{TCE} & \rightarrow \text { ethane } \\
& + \text { ethane }+\mathrm{VC} \\
& + \text { cis-DCE }\end{aligned}$ & $\begin{array}{l}\text { Solid-state } \\
\text { electrode } \\
\text { polarized at } \\
-500 \mathrm{mV}\end{array}$ & MV & Molar ratio of 1 & $\begin{array}{l}\text { Electrochemical reduction of MV promoted the dechlorination } \\
\text { of TCE, which did not occurred when MV was omitted on the } \\
\text { experiments }\end{array}$ & $\begin{array}{l}\text { Aulenta } \\
\text { et al. (2007) }\end{array}$ \\
\hline
\end{tabular}

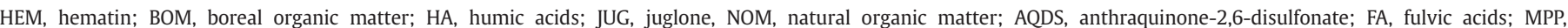

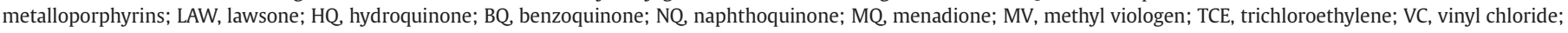
DCE, dichloroethylene; UP, unidentified products; RM, redox mediator.

a Palladized Zero Valent Iron.

b $\mathrm{C}_{2} \mathrm{Cl}_{4}$ added after 24 -h equilibrium between pd/ZVI and $\mathrm{HA}$.

obtained for the reduction of HCA was 3-fold higher at $50{ }^{\circ} \mathrm{C}$ (Curtis and Reinhard, 1994) compared to the reduction rate obtained at $25^{\circ} \mathrm{C}$ (Kappler and Haderlein, 2003).

\subsubsection{Redox potential}

As discussed above (azo dyes section), the $E_{0}{ }^{\prime}$ of a RM should ideally be in between those of the two eventual half reactions at stake,

Table 7

Impact of redox mediators on the microbial reduction of polychlorinated pollutants

\begin{tabular}{|c|c|c|c|c|c|}
\hline Reaction & Electron donor/system & Mediator & $\begin{array}{l}\text { Ratio pollutant/ } \\
\text { mediator }\end{array}$ & Results & References \\
\hline $\mathrm{CCl}_{4} \rightarrow \mathrm{CHCl}_{3}+\mathrm{CO}$ & $\begin{array}{l}\text { Lactate Shewanella alga } \\
\text { strain BrY }\end{array}$ & CNB12 & Molar ratio of 0.13 & $\begin{array}{l}\text { No conversion of CT by Shewanella alga } \\
\text { strain BrY in the absence CNB12; whereas } 92 \% \\
\text { of CT conversion to CO within } 3 \text { weeks of incubation }\end{array}$ & $\begin{array}{l}\text { Workman } \\
\text { et al. (1997) }\end{array}$ \\
\hline Octa-CDD $\rightarrow$ Tetra-CDD & $\begin{array}{l}\text { Contaminated sediment } \\
\text { without EED }\end{array}$ & $\begin{array}{l}\text { CAT, RES or } \\
\text { 3,4-DHBA }\end{array}$ & $\begin{array}{l}\text { Molar ratio of } \\
0.11\end{array}$ & $\begin{array}{l}\text { 4- to } 6 \text {-fold higher conversion in RM amended } \\
\text { cultures compared to control }\end{array}$ & $\begin{array}{l}\text { Barkovskii and } \\
\text { Adriaens } \\
\text { (1998) }\end{array}$ \\
\hline $\mathrm{CCl}_{4} \rightarrow \mathrm{CHCl}_{3}+\mathrm{UP}$ & $\begin{array}{l}\text { Lactate Shewanella } \\
\text { putrefaciens } 200\end{array}$ & NOM in soil & $\begin{array}{l}\sim 10 \times 10^{-} 3 \mu \mathrm{mol} \\
\mathrm{CCl}_{4} / \mathrm{mg} \mathrm{NOM}\end{array}$ & $\begin{array}{l}29 \% \text { of added } \mathrm{CT}(19.5 \mu \mathrm{M}) \text { converted after } 33 \mathrm{~h} \\
\text { in the absence of NOM; whereas } 64 \% \text { of } \mathrm{CCl}_{4} \\
\text { converted after } 18 \mathrm{~h} \text { in the presence of NOM }\end{array}$ & $\begin{array}{l}\text { Collins and } \\
\text { Picardal } \\
\text { (1999) }\end{array}$ \\
\hline $\mathrm{CCl}_{4} \rightarrow \mathrm{CHCl}_{3}+\mathrm{CO}_{2}+\mathrm{CS}_{2}$ & $\begin{array}{l}\text { Fructose Acetobacterium } \\
\text { woodii }\end{array}$ & HOB12 & Molar ratio of 47 & $\begin{array}{l}30 \text {-fold faster conversion rate in HOB12-amended } \\
\text { cultures compared to non-mediated incubations. } \\
\text { Addition of HOB12 also increased the extent of } \\
\text { mineralization (up to } 31 \% \text { measured as }{ }^{14} \mathrm{CO}_{2} \text { ) }\end{array}$ & $\begin{array}{l}\text { Hashsham } \\
\text { and Freedman } \\
\text { (1999) }\end{array}$ \\
\hline $\begin{array}{l}\mathrm{CCl}_{4} \rightarrow \mathrm{CHCl}_{3}+\mathrm{CH}_{2} \mathrm{Cl}_{2}+ \\
\quad \mathrm{PCE}+\mathrm{UP}\end{array}$ & $\begin{array}{l}\text { Anaerobic sludge or Geobacter } \\
s p \text {. provided with different } \\
\text { electron donors }\end{array}$ & AQDS or HA & $\begin{array}{l}\text { Molar ratio } \mathrm{CCl}_{4} / \mathrm{AQDS} \\
\text { of } 2 \text { to } 5 ; 0.05 \mu \mathrm{mol} \\
\mathrm{CCl}_{4} / \mathrm{mg} \mathrm{HA}\end{array}$ & $\begin{array}{l}\text { 6-fold higher first-order reduction rates in AQDS } \\
\text { amended sludge incubations compared to controls. } \\
\text { AQDS and HA stimulated the reduction of } \mathrm{CCl}_{4} \text { by Geobacter sp., } \\
\text { which did not convert } \mathrm{CCl}_{4} \text { in the absence of RM }\end{array}$ & $\begin{array}{l}\text { Cervantes } \\
\text { et al. (2004) }\end{array}$ \\
\hline $\mathrm{CCl}_{4} \rightarrow \mathrm{CHCl}_{3}+\mathrm{Cl}^{-}+\mathrm{UP}$ & $\begin{array}{l}\text { Anaerobic sludge provided } \\
\text { with VFA }\end{array}$ & $\begin{array}{l}\text { AQDS, RF, } \\
\text { CNB12, } \\
\text { HOB12 }\end{array}$ & Molar ratio of 10 & $\begin{array}{l}3.8-, 4.0,13.3-\text { and } 13.6 \text {-fold higher first-order reduction } \\
\text { rates in AQDS, RF, CNB12 and HOB12 amended cultures, } \\
\text { respectively, compared to controls }\end{array}$ & $\begin{array}{l}\text { Guerrero- } \\
\text { Barajas and } \\
\text { Field (2005) }\end{array}$ \\
\hline
\end{tabular}

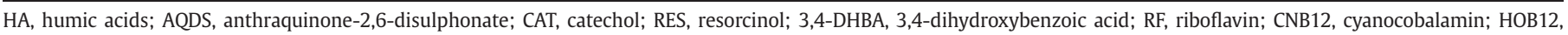
hydroxycobalamin; UP, unidentified products; CDD, chlorinated dibenzo-p-dioxins, RM, redox mediator; VFA, volatile fatty acids; EED, external electron donor. 
the reduction of an electron-withdrawing pollutant and the oxidation of a primary electron donor.

There are several reports indicating the relationship between the $E_{0}^{\prime}$ of the RM and the dechlorinating rate observed for different PCC. During the reductive dechlorination of CT by ZVI, for instance, AQDS increased the reduction rate of $\mathrm{CT}$ at a higher extent (up to 3-fold higher than non-mediated control) compared to the catalytic effects of juglone (up to 2.2-fold higher than non-mediated control) (Tratnyek et al., 2001). The last correlation is expected as AQDS has a more negative $E_{0}^{\prime}$ compared to juglone. Comparable results were reported during the reductive dechlorination of perchloroethylene (PCE) by ZVI in the presence of different quinoid RM (Doong and Lai, 2005). Hydroquinone, with the lowest $E_{0}^{\prime}$, promoted the maximum reduction rates of PCE by ZVI; whereas $p$-benzoquinone, with the highest $E_{0}^{\prime}$, caused the lowest reduction rates for PCE.

Biological dechlorination rates catalyzed by distinct RM also showed a high correlation between the $E_{0}$ of the RM and the reductive dechlorinating rates. The last scenario occurred when AQDS, riboflavin (RF), and cyanocobalamin (CNB12) were tested as RM during the anaerobic reduction of CT by anaerobic sludge (Guerrero-Barajas and Field, 2005). The observed development of reduction rates of CT was consistent with a decreasing trend of $E_{0}^{\prime}$ of the RM tested. CNB12, which has the most negative $E_{0}^{\prime}(-530 \mathrm{mV})$ enhanced 13.3-fold the reduction rate of $\mathrm{CT}$, compared to the non-mediated control; whereas $\mathrm{RF}\left(E_{0}^{\prime}\right.$ of $-208 \mathrm{mV}$ ) and AQDS ( $E_{0}^{\prime}$ of $-184 \mathrm{mV}$ )increased the reduction rate of CT by 4- and 3.7-fold, respectively (Guerrero-Barajas and Field, 2005).

Despite all these lines of evidence, it is important to emphasize that a change in $E_{0}^{\prime}$ of the RM does not always reflect a direct correlation with dechlorinating kinetics. For example, the reduction rate of CT by sulfide, in the presence of different quinoid RM, was evaluated as a function of the $E_{0}^{\prime}$ of the quinones tested (Doong and Chiang, 2005). Juglone (JQ), naphthoquinone (NQ) and benzoquinone (BQ) were found to be more effective electron-transfer mediator than AQDS during the reductive transformation of CT by sulfide. The reduction rates observed in the presence of JQ and NQ were 10-14 higher than those obtained with AQDS, despite that the driving force for CT transformation could be expected to be greater for AQDS than that for $\mathrm{NQ}$ based on the $E_{0}^{\prime}$ values. Moreover, JQ NQ and BQ increased the reduction rate of $\mathrm{CT}$ a greater extent compared to the catalytic effects of AQDS and menadione (MQ), which also contrasts with the previously reported correlation between the $E_{0}^{\prime}$ values and reductive transformation rates (Tratnyek et al., 2001; Rau et al., 2002; Doong and Lai, 2005; Guerrero-Barajas and Field, 2005). The last results mean that the $E_{0}^{\prime}$ of quinoid RM may not be the only factor that controls the reduction rate of PCC, and that the chemical structures may play an important role in determining the reactivity of quinoid compounds (Doong and Chiang, 2005).

The reduction rate of an electron-withdrawing pollutant by a reduced RM would theoretically increase with decreasing $E_{0}^{\prime}$ of the $\mathrm{RM}$; but the reduction rate of the RM by sulfide will decrease with decreasing $E_{0}^{\prime}$. The last observation may explain the lower catalytic input of AQDS on CT dechlorination, compared to the catalysis observed with BQ and JQ (Doong and Chiang, 2005). Nevertheless, according to the same hypothesis, dechlorination of $C T$ would proceed faster by previously reduced AQDS than by reduced BQ or JQ.

\subsubsection{Reactive species involved in reductive dechlorination reactions}

Different reactive species including semiquinone, hydroquinone, and mercaptoquinone have been shown to successfully transfer electrons from bulk reductants to electron-withdrawing organic pollutants (Schwarzenbach et al., 1990; Curtis and Reinhard, 1994; Perlinger et al., 1996). The mechanism of formation of reactive species during reductive transformation processes has been linked to the characteristic redox cycling properties of the quinone compounds (Doong and Chiang, 2005).
Experimental evidence suggests that the concentration of semiquinone radicals, produced during reductive transformations, controls the rate of conversion of organic pollutants (Scott et al., 1998; Rau et al., 2002). Further research indicates that the amounts of deprotonated hydroquinone and semiquinone radicals are correlated to the degradation efficiency of priority pollutants in AQDS- and HAamended incubations under reducing conditions (Tratnyek and Macalady, 1989; Curtis and Reinhard, 1994; Scott et al., 1998). Monophenolate (Schwarzenbach et al., 1990) and mercaptojuglone (Perlinger et al., 1996) have also been identified as the dominant reactive species during reductive transformations mediated by JQ in the presence of sulfide as electron donor. However, the rate of degradation of HCA was more specifically linked to the production of mercaptojuglone in its hydroquinone or semiquinone form at different pH values (Perlinger et al., 2002). A preliminary report elucidated the mechanism by which JQ might decrease the Ea in dechlorination reaction with sulfide as electron donor. Mercaptojuglone is the reactive species readily formed in incubation assays including both sulfide and JQ (Perlinger et al., 1998). Mercaptojuglone enhanced the reductive dechlorination of different PCC at a higher extent than that expected by the input of reduced JQ alone (Perlinger et al., 1998).

More recently, results derived from electron paramagnetic resonance, UV-vis spectroscopy and electrospray ionization mass spectroscopy of different quinoid RM, clearly showed that three groups of quinones can be categorized based on the formation of the active electron-transfer mediators involved in the reductive transformation of CT (Doong and Chiang, 2005). JQ, BQ and NQ produced mercaptoquinones as reactive species, which enhanced up to 71-fold the reduction rate of $\mathrm{CT}$, compared to the control lacking quinones. In contrast, MQ and LQ, which were reduced to their corresponding hydroquinone by sulfide, promoted a much lower enhancement on the reduction rate of CT. Moreover, reduction of AQDS by sulfide produced semiquinone radicals, which moderately increased the reduction rate of CT. Therefore, the catalytic effects of different quinoid RM strongly depends on the type of reactive species produced during reductive transformation processes. Mercaptoquinone is a more efficient reactive species than either semiquinone radicals or hydroquinone for transferring electrons in a reducing environment containing thiol reductants (Doong and Chiang, 2005). The last observations could explain the remarkably higher dechlorination rate of CT promoted by LQ compared to reactions mediated by AQDS, despite the lower $E_{0}{ }^{\prime}$ of the former RM (Doong and Chiang, 2005).

\subsubsection{Variety of electron donors and natural sources of RM}

Two half-reactions are at stake during reductive dechlorinations mediated by RM: 1) reduction of redox active groups by a proper electron donor; and 2) subsequent reductive dechlorination of PCC by reduced RM (Fig. 1). Therefore, the overall reaction rate depends on the capacity of the external electron donor to transfer electrons to the RM and on the ability of the reduced RM to channel the electrons to PCC. According to the experimental data available in the literature, the reduction of different $\mathrm{RM}$, by chemical and biological processes, proceeds generally faster than the subsequent transfer of electrons from the reduced RM to PCC. For instance, the reduction of AQDS and HA by sulfide occurs within a few hours (Dunnivant et al., 1992; Cervantes, unpublished results), whereas reductive dechlorinations of different PCC by reduced AQDS or HA demand several days of incubation (Curtis and Reinhard, 1994; Kappler and Haderlein, 2003; Cervantes et al., 2004). A wide variety of microorganisms, including iron-reducing, sulfate-reducing, halorespiring, fermentative and methanogenic microorganisms, are also capable of rapidly reducing HA or quinones, which could then be available to promote reductive dechlorinations in anaerobic environments (Field and Cervantes, 2005). Ferrous iron is another reductant commonly found in anaerobic environments, but its reducing capacity is minor compared to sulfide, 
which is reflected by the lower dechlorination rates achieved with the former (Curtis and Reinhard, 1994).

For biologically mediated dechlorinations, glucose, formate and hydrogen generally promote higher dechlorination rates, compared to those obtained with methanol and acetate, which agrees with the faster quinone-reducing activities observed with the former substrates (Cervantes et al., 2000a,b; 2004; Guerrero-Barajas and Field, 2005).

Many different model RM, such as quinoid- and flavin-based compounds, have a higher solubility compared to natural sources of RM (e.g. humic materials), which may also imply different catalytic inputs during reductive dechlorinations. It is conceivable that RM with a higher solubility could be more easily reduced, either chemically or biologically, compared to RM remaining suspended on the reaction system, which in turn would promote faster dechlorination rates. The last observation explains the higher dechlorination rates generally achieved with soluble model RM than with suspended NOM (Schwarzenbach et al., 1990; Dunnivant et al., 1992; Tratnyek et al., 2001). Furthermore, suspended NOM could even inhibit dechlorination reactions when insoluble electron donors participate in dechlorination reactions. For example, the presence of suspended NOM in dechlorination assays, with ZVI as an electron donor, decreased the rate and extent of dechlorination of trichloroethylene (TCE) and PCE by competitive sorption onto reactive sites of ZVI surface (Tratnyek et al., 2001; Doong and Lai, 2005). In contrast, different soluble RM, such as AQDS and LQ increased the rate and extent of dechlorination of different PCC by ZVI (Tratnyek et al., 2001; Doong and Lai, 2005).

The content of redox active groups in NOM also plays a role on determining the catalytic input of RM in dechlorination reactions. For instance, the reductive transformation of CT by Shewanella putrefaciens 200 was enhanced to a greater extent by HA compared to CT dechlorination mediated by fulvic acids (FA) (Collins and Picardal, 1999). A larger content of quinones has been reported in HA compared to FA (Stevenson, 1994), which may explain the last phenomenon. Nevertheless, despite the inherent variations in concentration, accessibility, and reactivity of redox-active groups in HA originated from a variety of environments, the capacity of electrochemically reduced HA to achieve the abiotic dechlorination of PCC was fairly constant (Kappler and Haderlein, 2003).

\section{Role of redox mediators on the reduction of nitroaromatic pollutants}

\subsection{Introduction}

Mono- and polynitroaromatic compounds (PNACs) are widely used as agrochemicals, explosives, textile dyes, and as chemical intermediates for different manufacturing sectors. Due to their widespread use and improper disposal, contamination of soils, sediments and groundwater by PNACs is a ubiquitous problem. Several reports have confirmed the toxic and adverse effects of PNACs on biological ecosystems and on human health. Consequently, the restoration of PNACs-contaminated sites is a matter of concern among environmental protection groups (Hofstetter et al., 1999).

Tables 8 and 9 recapitulate the experimental data obtained during the reductive (bio)transformation of PNACs mediated by different RM. Most reports deal with the abiotic transformation of PNACs illustrating the catalytic effects of model RM or NOM from different origins (Table 8). Recently, reductive biotransformation of PNACs by distinct microorganisms has also been reported (Table 9). In this section the input of different RM on the reductive (bio)transformation of PNACs will be discussed, as well as the different aspects governing the catalytic reactions.

\subsection{Main factors determining the input of $R M$ in the reduction of nitroaromatic pollutants}

\subsection{1. $\mathrm{pH}$}

Several studies have documented the effect of $\mathrm{pH}$ during reductive transformation of PNACs mediated by different redox reactive groups.

Table 8

Impact of redox mediators on reduction of nitroaromatic compounds under abiotic conditions

\begin{tabular}{|c|c|c|c|c|c|}
\hline Reaction & $\begin{array}{l}\text { Electron } \\
\text { donor/system }\end{array}$ & Mediator & Ratio pollutant/mediator & Results & References \\
\hline $\begin{array}{l}\text { Various } \\
\quad \text { nitroaromatics }{ }^{\mathrm{a}} \rightarrow \text { Anilines }\end{array}$ & $\begin{array}{l}\text { Sulfide or } \\
\text { cysteine }\end{array}$ & $\begin{array}{l}\text { JUG, } \\
\text { LAW or } \\
\text { IPP }\end{array}$ & Molar ratio of 0.4 to 50 & $\begin{array}{l}\text { Second-order rate constants for the reduction of } \\
\text { nitroaromatics between } 3.0 \times 10^{-3} \text { and } 3.3 \times 10^{2} \mathrm{M}^{-1} \mathrm{~s}^{-1} \text { in } \\
\text { the presence of RM, whereas no significant reduction } \\
\text { occurred in the absence of RM }\end{array}$ & $\begin{array}{l}\text { Schwarzenbach } \\
\text { et al. (1990) }\end{array}$ \\
\hline $\begin{array}{l}\text { Various } \\
\qquad \text { nitroaromatics }{ }^{\mathrm{b}} \rightarrow \text { Anilines }\end{array}$ & Sulfide & $\begin{array}{l}\text { NOM } \\
\text { from } \\
\text { different } \\
\text { origins }\end{array}$ & $1.21 \mu \mathrm{mol} \mathrm{NA} / \mathrm{mg}$ C-NOM & $\begin{array}{l}\text { Second-order rate constants for the reduction of } \\
\text { nitroaromatics between } 3.0 \times 10^{-6} \text { and } 3.3 \times 10^{-2} \mathrm{~h}^{-1}(\mathrm{mg} \mathrm{C} \text { - } \\
\mathrm{NOM} / \mathrm{L})^{-1} \text { in the presence of NOM, whereas no significant } \\
\text { reduction occurred in the absence of NOM at pH of } 7.2\end{array}$ & $\begin{array}{l}\text { Dunnivant et al. } \\
\text { (1992) }\end{array}$ \\
\hline $\begin{array}{l}\text { Various } 2,6- \\
\text { DNAH } \rightarrow \text { corresponding } \\
\text { anilines }\end{array}$ & ZVI & $\begin{array}{l}\text { HA or } \\
\text { dichlone }\end{array}$ & $0.5 \mu \mathrm{mol} 2,6-\mathrm{DNAH} / \mathrm{mg} \mathrm{HA}$ & $\begin{array}{l}\text { HA amendments decreased the first-order rate constant of } \\
\text { reduction of 2,6-DNAH for 1.2-10 fold compared to controls } \\
\text { lacking HA. Dichlone amendments counteracted the } \\
\text { negative effects of HA achieving similar reduction rates to } \\
\text { the control lacking RM }\end{array}$ & $\begin{array}{l}\text { Keum and Li } \\
\text { (2004) }\end{array}$ \\
\hline $\mathrm{PCNB} \rightarrow \mathrm{PCA}$ & $\mathrm{Fe}(\mathrm{II})$ & $\begin{array}{l}\text { DOM } \\
\text { from } \\
\text { different } \\
\text { origins }\end{array}$ & 0.06 to $0.1 \mu \mathrm{mol} \mathrm{PCNB} / \mathrm{mg}$ C-DOC & $\begin{array}{l}\text { First-order rate constants of reduction of PCNB up to } \sim 8 \text {-fold } \\
\text { higher in DOC-amended assays compared to controls } \\
\text { without DOC. Unfiltered NOM inhibited the reductive } \\
\text { process }\end{array}$ & $\begin{array}{l}\text { Hakala et al. } \\
\text { (2007) }\end{array}$ \\
\hline $\begin{array}{l}\text { Various } \\
\qquad \text { nitroaromatics }^{\mathrm{d}} \rightarrow \text { Anilines }\end{array}$ & Sulfide & JUG & NA & $\begin{array}{l}\text { Second-order rate constants for the reduction of } \\
\text { nitroaromatics between } 2.3 \times 10^{-3} \text { and } 6.9 \times 10^{3} \mathrm{M}^{-1} \mathrm{~s}^{-1} \text { in } \\
\text { the presence of JUG, whereas no significant reduction } \\
\text { occurred in the absence of JUG }\end{array}$ & $\begin{array}{l}\text { Hofstetter et al. } \\
\text { (1999) }\end{array}$ \\
\hline $\mathrm{NB} \rightarrow$ Aniline & ZVI & $\begin{array}{l}\text { FA, AQDS } \\
\text { or LAW }\end{array}$ & $\begin{array}{l}12.5 \text { to } 50 \mu \mathrm{mol} \mathrm{NB} / \mathrm{mg} \mathrm{FA} \text {; molar } \\
\text { ratio of } 2.5 \text { and } 4.4 \text { for AQDS and } \\
\text { LAW amended incubations, } \\
\text { respectively }\end{array}$ & $\begin{array}{l}\text { Up to } 20 \% \text { decrease on first-order rate constants of reduction } \\
\text { of NB in FA-amended assays; whereas a } 1.2 \text { - and 1.52-fold } \\
\text { increase was observed in LAW- and AQDS-amended assays }\end{array}$ & $\begin{array}{l}\text { Tratnyek et al. } \\
(2001)\end{array}$ \\
\hline
\end{tabular}

\footnotetext{
a Including NB, 2-, 3- and 4-methyl-NB, 2-, 3- and 4-chloro-NB, 2-, 3- and 4-acetyl-NB.
}

b Including the same nitroaromatics considered by Schwarzenbach et al. (1990).

c Including benfluralin, ethalfluralin, nitralin, oryzalin, pendimethali and trifluralin.

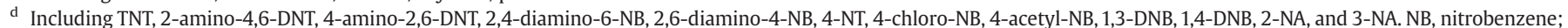

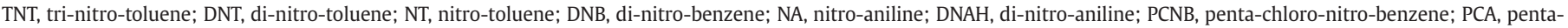

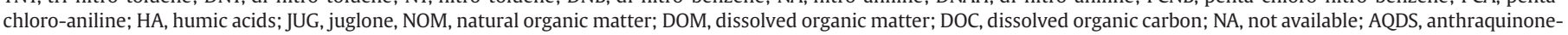
2,6-sulphonate; FA, fulvic acids; IPP, ironporphyrin; LAW, lawsone; RM, redox mediator. 
Table 9

Impact of redox mediators on the microbial reduction of nitroaromatic compounds

\begin{tabular}{|c|c|c|c|c|c|}
\hline Reaction & Electron donor/system & Mediator & Ratio pollutant/mediator & Results & References \\
\hline $\begin{array}{l}\text { TNT } \rightarrow 2,4-D H A N T \text { and } \\
\text { UP }\end{array}$ & $\begin{array}{l}\text { Sucrose/Cellulomonas sp. } \\
\text { strain ES6 }\end{array}$ & AQDS & Molar ratio TNT/AQDS between 0.06 and 6 & $\begin{array}{l}\text { Up to 3.7-fold increase in first-order rate } \\
\text { constant of reduction of TNT in AQDS-amended } \\
\text { assays compared to controls lacking AQDS }\end{array}$ & $\begin{array}{l}\text { Borch } \\
\text { et al. (2005) }\end{array}$ \\
\hline $\begin{array}{l}\text { RDX or HMX } \rightarrow \text { nitrite, } \\
\text { MDNA, nitrous oxide, } \\
\text { ammonium, } \\
\text { formaldehyde, } \\
\text { formic acid and } \mathrm{CO}_{2}\end{array}$ & $\begin{array}{l}\text { Glucose/Clostridium sp. } \\
\text { EDB2 }\end{array}$ & $\begin{array}{l}\text { HA or } \\
\text { AQDS }\end{array}$ & $\begin{array}{l}\text { Molar ratio RDX/AQDS of } 0.075 \text { to } 1.5 \text {; molar ratio } \\
\text { HMX/AQDS of } 0.0275 \text { to } 0.55 \text {. For HA-amended } \\
\text { assays the ratio RDX/HA and HMX/HA were } \\
0.075 \text { to } 1.5 \text { and } 0.0275 \text { to } 0.55 \mu \mathrm{mol} / \mathrm{g} \text {, } \\
\text { respectively }\end{array}$ & $\begin{array}{l}\text { Complete removal of RDX in } 5 \text { and } 8 \text { days in the } \\
\text { presence of HA and AQDS, respectively, } \\
\text { compared to } 10 \text { days required in unamended } \\
\text { controls; } 28-60 \% \text { higher mineralization of RDX in } \\
\text { HA- and AQDS-amended assays compared to } \\
\text { controls. Similar results obtained with HMX. }\end{array}$ & $\begin{array}{l}\text { Bhushan } \\
\text { et al. (2006) }\end{array}$ \\
\hline $\mathrm{RDX} \rightarrow \mathrm{TNX}$ & $\begin{array}{l}\text { Acetate-PCIO/Geobacter } \\
\text { metallireducens strain } \\
\text { GS-15 or Geobacter } \\
\text { sulfurreducens strain PCA }\end{array}$ & $\begin{array}{l}\text { HA or } \\
\text { AQDS }\end{array}$ & $\begin{array}{l}\text { Molar ratio RDX/AQDS of } 8 \times 10^{-3} \text {. For } \\
\text { HA-amended assays the ratio RDX/HA was } \\
160 \mu \mathrm{mol} / \mathrm{g}\end{array}$ & $\begin{array}{l}\text { Addition of HA or AQDS to Fe(III)-containing } \\
\text { microbial cultures increased 5- to } 66 \text {-fold the } \\
\text { reduction rate of RDX compared to unamended } \\
\text { controls }\end{array}$ & $\begin{array}{l}\text { Kwon and } \\
\text { Finneran } \\
(2006)\end{array}$ \\
\hline $\begin{array}{l}\mathrm{RDX} \rightarrow \text { nitrite, MDNA, } \\
\text { nitrous oxide, } \\
\text { ammonium, } \\
\text { formaldehyde, and } \\
\mathrm{CO}_{2}\end{array}$ & $\begin{array}{l}\text { Acetate/Geobacter } \\
\text { metallireducens }\end{array}$ & AQDS & NA & $\begin{array}{l}\text { Addition of AQDS increased the rate and extent } \\
\text { of mineralization of RDX by Geobacter } \\
\text { metallireducens }\end{array}$ & $\begin{array}{l}\text { Kwon and } \\
\text { Finneran } \\
\text { (2008) }\end{array}$ \\
\hline
\end{tabular}

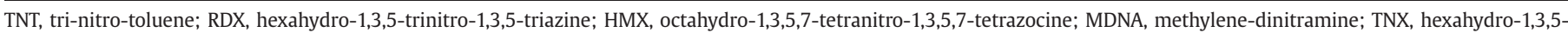

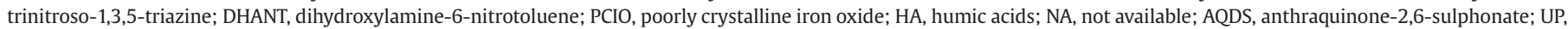
unidentified products.

The first detailed description came after the study of Tratnyek and Macalady (1989), who evaluated the capacity of different reduced quinone moieties to reduce methyl parathion over the $\mathrm{pH}$ range between 5.5 and 9.0. Reduction of this nitroaromatic pesticide by reduced indigodisulfonate proceeded at the fastest rate at $\mathrm{pH}$ around 7.1, but declined rapidly on both sides of this maximum. Similar results were obtained during the reduction of methyl parathion by $\mathrm{AH}_{2} \mathrm{QDS}$, but the maximum reduction rate occurred at a $\mathrm{pH}$ around 6.0. Further inspection of the reaction systems, based on $E_{0}^{\prime}-\mathrm{pH}$ diagrams for both $\mathrm{RM}$, revealed that the concentration of the hydroquinone with one of the hydroxyl groups dissociated (monophenolate form) is substantial at $E_{0}^{\prime} / \mathrm{pH}$ conditions at which the highest reduction rate of methyl parathion occurred. Based on these observations, the authors developed a connection model between deprotonation of -OH groups of the RM and the reduction rate of methyl parathion. A high correlation between the reduction rate of methyl parathion and the calculated concentration of monophenolate species of both RM was obtained (Tratnyek and Macalady, 1989).

The same deprotonation model was used by Schwarzenbach et al. (1990) to describe the effect of $\mathrm{pH}$ on the reduction of different PNACs by sulfide or cysteine, mediated by quinoid RM. The authors proposed the following equation to calculate the reduction rate of PNACs as a function of the concentration of the monophenolate ([HQ-]) and biphenolate ([Q-2]) species of the reduced RM:

$k_{\mathrm{obs}}=k_{\mathrm{HQ}-}\left[\mathrm{HQ}^{-}\right]+k_{\mathrm{Q}-2}\left[\mathrm{Q}^{-2}\right]$

where $k_{\mathrm{HQ}}$ and $k_{\mathrm{Q}-2}$ are the second-order rate constant for the reaction of the nitroaromatic compound with the hydroquinone monophenolate and biphenolate, respectively. Non-dissociated hydroquinone species were negligibly reactive, as compared to the dissociated species, and therefore were not considered in the equation. Experimental data revealed that for the 2 quinoid RM evaluated (JQ and LQ), the biphenolate species was $\sim 500$-fold more reactive than the monophenolate species. Thus, $\mathrm{pH}$ changes, particularly at $\mathrm{pH}$ values above 7 , have a dramatic impact on the reduction rate of PNACs.

Furthermore, a somewhat more complex $\mathrm{pH}$ dependence of the reaction rates was found for the reduction of nitrophenols, as these compounds are weak acids, and may thus, be present in both the nondissociated and the dissociated forms. Evidence indicated that the non-dissociated species of several nitrophenols studied reacted $\sim 3$ fold faster with the monophenolate form of reduced LQ than their dissociated species (Schwarzenbach et al., 1990).
Similar $\mathrm{pH}$ dependence was also observed during the reductive transformation of 3-chloro-nitrobenzene by sulfide, mediated by NOM from different origins (Dunnivant et al., 1992), corroborating the higher reactivity of dissociated quinoid-redox groups in NOM, compared to that of non-dissociated species.

In a more recent study, the effect of $\mathrm{pH}$ on the rate of reduction of hexahydro-1,3,5-trinitro-1,3,5-triazine (RDX) by $\mathrm{AH}_{2} \mathrm{QDS}$ was investigated (Kwon and Finneran, 2008). As the pH increased from 6.2 to 9.2 at constant $\mathrm{AH}_{2} \mathrm{QDS}$ concentration $(100 \mu \mathrm{M})$, the reduction rate of $\mathrm{RDX}$ increased 61-fold. Furthermore, the authors found that the reduction rate of RDX was very closely related to the concentration of the monophenolate hydroquinone. In addition, results obtained with cell suspensions of Geobacter metallireducens degrading RDX, showed a decrease on the reduction rate of this contaminant by raising the $\mathrm{pH}$ in the microbial incubations in the absence of AQDS. Nevertheless, in AQDS-amended cell incubations of G. metallireducens, the reduction rate of RDX was significantly increased, compared to the reaction rates observed without AQDS, but remained similar within the whole $\mathrm{pH}$ range tested (6.8, 7.9 and 9.2) (Kwon and Finneran, 2008).

\subsubsection{Redox potential}

The reduction of several nitroaromatic pollutants under different redox conditions has been investigated. The effect of the $E_{0}^{\prime}$ of a model system, including the reduced form of either indigodisulfonate or AQDS, on the rate of reduction of methyl parathion was studied (Tratnyek and Macalady, 1989). Experiments were conducted at a $E_{0}^{\prime}$ range between -37 to $-208 \mathrm{mV}$, and -90 to $-140 \mathrm{mV}$, for indigodisulfonate- and AQDS-supplied incubations, respectively. The results showed that the reduction rate of the nitroaromatic pesticide increased as $E_{0}^{\prime}$ became more negative with both quinones evaluated. This trend observed is reasonable insofar as a low $E_{0}^{\prime}$ reflects a high concentration of reduced species in the reaction system, which are then available to promote the reduction of methyl parathion.

Further studies also revealed a high correlation between the $E_{0}^{\prime}$ of the reaction system and the rate of reduction of 3-chloro-nitrobenzene by different concentrations of sulfide in the presence of different sources of NOM (Dunnivant et al., 1992). As expected, a more negative value of $E_{0}^{\prime}$, achieved by a higher concentration of sulfide, promoted a higher reduction rate of the nitroaromatic pollutant. Moreover, the authors compared the capacity of reduced NOM, obtained by bubbling hydrogen into platinum-amended solutions, with the incubations described above. The results indicated slightly lower reduction rates than those obtained with sulfide, but revealed that reduced functional 
groups of NOM, independent of the reduction method employed, are capable of mediating the reduction of nitroaromatic compounds. The increased reduction rate of 3-chloro-nitrobenzene observed by increasing the $E_{0}^{\prime}$ was attributed to the additional reduction of reactive functional groups in NOM exhibiting a more negative reduction potential.

The reduction of nitrobenzene by ZVI was explored in the presence of different RM (Tratnyek et al., 2001). The obtained results also indicate the influence of the $E_{0}^{\prime}$ on the reaction rate. Indeed, AQDS, which has a more negative $E_{0}^{\prime}(-184 \mathrm{mV})$ than LQ $(-137 \mathrm{mV})$, promoted a higher reduction rate of nitrobenzene than the latter RM.

\subsubsection{Biodegradation pathway}

Although limited studies have been conducted to elucidate the impact of RM on the reduction of PNACs by microorganisms, the literature reveals that RM not only accelerate the reduction of PNACs, but also have a remarkable effect on the biodegradation pathway (Borch et al., 2005; Bhushan et al., 2006; Kwon and Finneran, 2006; Kwon and Finneran, 2008).
The reduction rate of 2,4,6-trinitrotoluene (TNT) by Cellulomonas sp. strain ES6 was increased up to 3.6-fold by the addition of different concentrations of AQDS (10-1000 $\mu \mathrm{M}$, (Borch et al., 2005). Hydroxylaminodinitrotoluenes, 2,4-dihydroxylamino-6-nitrotoluene (2,4DHANT), and tetranitroazoxytoluenes were the major metabolites observed in pure cultures of strain ES6 in the absence of AQDS. The presence of AQDS promoted the rapid initial formation of arylhydroxylamines, such as 2,4-DHANT, which were further transformed into several unidentified polar products. Moreover, results obtained in the presence of strain ES6, ferrihydrite and $100 \mu \mathrm{M}$ AQDS showed further alterations on the TNT biodegradation pathway. Increased reduction rates of 4-hydroxyaminodinitrotoluene (2-HADNT) and 4-aminodinitrotoluene (4-ADNT) to 2,4-diaminonitrotoluene (2,4-DANT) were observed (Steps 1.1, 6 and 8, Fig. 3). Only traces of 2-HADNT and 2,4DHANT were observed, whereas 2,4-DANT accounted for up to $79 \%$ of the mass balance at the end of the incubation period $(70 \mathrm{~h})$. The input of AQDS on ferrihydrite-amended cultures was explained by an increased concentration of surface-associated redox active $\mathrm{Fe}(\mathrm{II})$, promoted by AQDS, which then enhanced the Fe(II)-mediated TNT reduction (Borch et al., 2005).

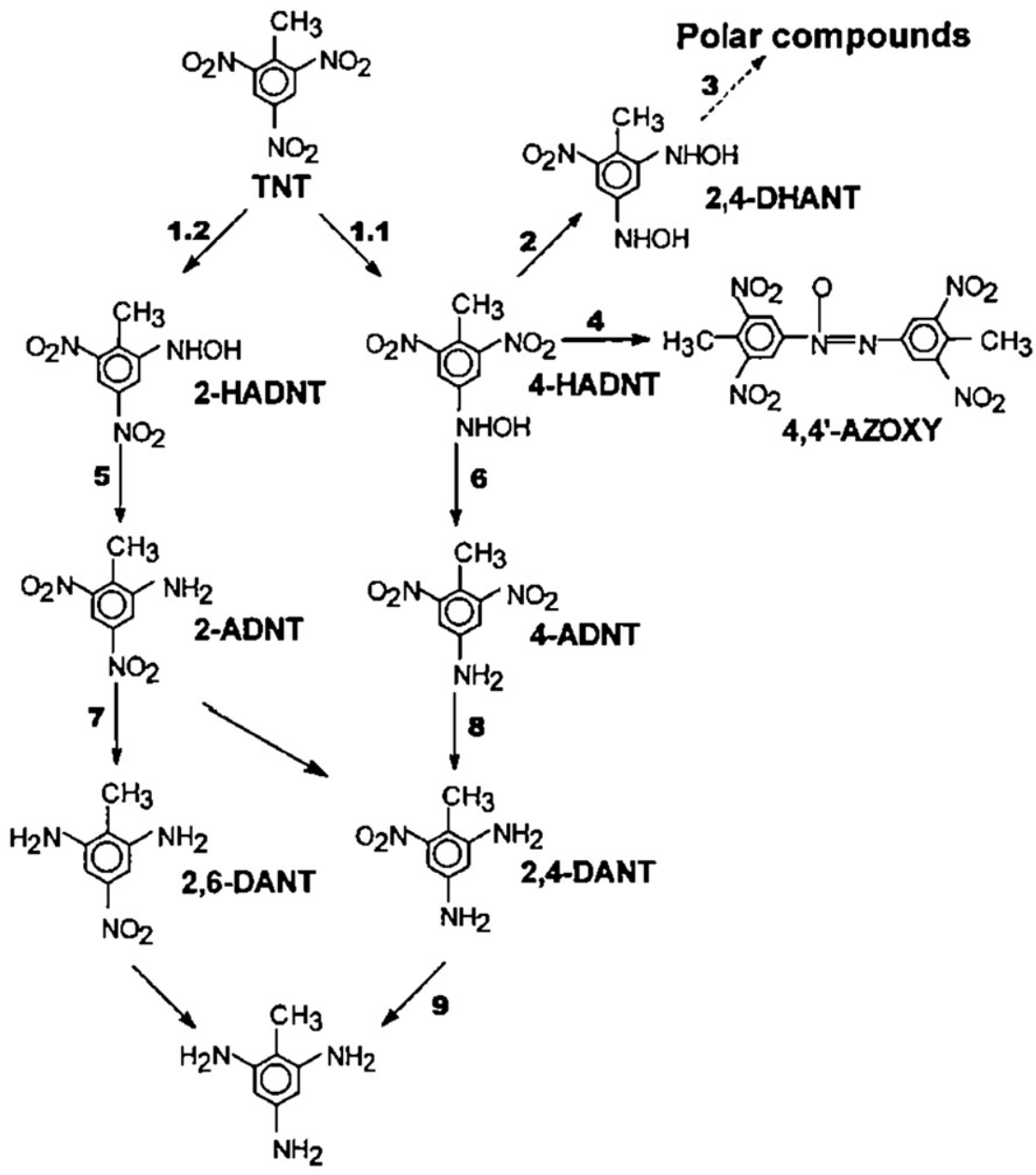

TAT
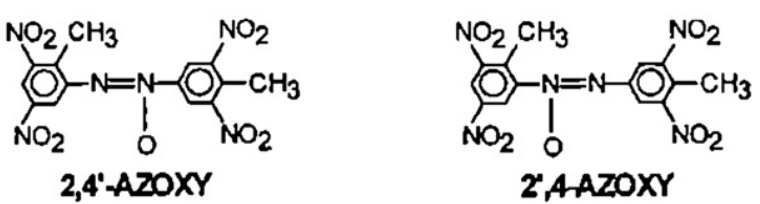

Fig. 3. Pathways and metabolites observed during the AQDS-mediated degradation of TNT by Cellulomonas sp. strain ES6 (after Borch et al., 2005). 
The catalytic effects of HA and AQDS on the biodegradation of hexahydro-1,3,5-trinitro-1,3,5-triazine (RDX) and octahydro-1,3,5,7tetranitro-1,3,5,7-tetrazocine (HMX) by Clostridium sp. strain EDB2 was studied (Bhushan et al., 2006). The experimental data revealed that the presence of either HA or AQDS, in cell suspensions of strain EDB2 degrading these cyclic nitramines, not only increased the biotransformation rates, but also the extent of mineralization of both pollutants. Up to $40 \%$ of uniformly labeled ${ }^{14} \mathrm{C}$-RDX $(150 \mu \mathrm{M})$ was mineralized to ${ }^{14} \mathrm{CO}_{2}$ when microbial incubations were performed in the presence of HA or AQDS; whereas only $28 \%$ of mineralization occurred in the absence of RM after 45 days of incubation. Moreover, of the initial amount of uniformly labeled ${ }^{14} \mathrm{C}-\mathrm{HMX}(55 \mu \mathrm{M})$ added to microbial incubations of strain EDB2, only 5\% was mineralized to ${ }^{14} \mathrm{CO}_{2}$ in the absence of $\mathrm{RM}$, while $22 \%$ of mineralization was detected in both HA- and AQDS-amended cultures (Bhushan et al., 2006).

In a recent study, the impact of AQDS on the biodegradation of RDX by $G$. metallireducens was evaluated (Kwon and Finneran, 2008). In abiotic incubations including $\mathrm{AH}_{2} \mathrm{QDS}$ as a sole electron donor, for the reduction of uniformly labeled ${ }^{14} \mathrm{C}-\mathrm{RDX}$, the main products obtained were methylenedinitramine (MEDINA), formaldehyde, nitrous oxide, nitrite and ammonium, but no ${ }^{14} \mathrm{CO}_{2}$ was detected. During the incubation of $G$. metallireducens in the absence of AQDS, formaldehyde was the primary carbon metabolite produced and hexahydro-1nitroso-3,5-dinitro-1,3,5-triazine (MNX) did not accumulate. However, MEDINA was less significant compared to the level detected in abiotic conditions; whereas ammonium appeared as the main nitrogenous product. The reduction of RDX by G. metallireducens was significantly increased in the presence of AQDS and formaldehyde and MEDINA were concurrently produced; however, MEDINA was further metabolized leading to a greater extent of mineralization evidenced by a higher production of ${ }^{14} \mathrm{CO}_{2}$ (Kwon and Finneran, 2008).

\section{Role of redox mediators in miscellaneous processes}

\subsection{Introduction}

The reactions dealt with so far were all reductive (bio)transformations of organic pollutants, and RM-catalysis of these reactions was based on a mostly extracellular reaction between a chemically or biologically reduced mediator with the organic electron-accepting pollutant. A similar mechanism is responsible for RM-catalysis of the reduction of vat and anthraquinone dyes (Section 5.2). RM may also play a role in the biological reduction of (bulk) inorganic electron acceptors such as nitrate, perchlorate and arsenate, through a mechanism that involves biological oxidation instead of reduction of the external RM (Section 5.2). In addition, there are other types of both biological and chemical oxidation-reduction processes that depend on or are accelerated by redox mediators. Important examples of such processes are the (dissimilatory) reduction of oxidized metal minerals (e.g. ferric iron) and anode reduction in microbial fuel cells (MFC), where RM serve to facilitate the transport of electrons respectively from and to solid materials (Section 5.3). Moreover, inversely analogous to anode reduction, also cathode oxidation can be catalyzed by RM (Section 5.4). Another type of processes that can be accelerated by RM is the chemical or biological oxidation of bulk reductants such as sulfide (Section 5.5). Furthermore, RM are known to catalyze enzymatic oxidation of organic compounds (Section 5.6). Most of these RM-catalyzed processes are beyond the scope of this paper and have already received ample attention in other review articles. In the sections below we will only briefly discuss these topics, wherever possible with reference to relevant review papers.

\subsection{Reduction of vat and anthraquinone dyes}

Redox mediators have been shown to accelerate the reduction of two classes of non-azo dyes, vat dyes and anthraquinone dyes. The principle of vat dyeing is sequential reduction and oxidation. In the reduction step, vat dyes such as indigo are solubilized (and often decolorized), and in the consecutive oxidation step, the dyes regain their color and precipitate on the fabric. It has been demonstrated that quinones can speed up the chemical reduction of vat dyes by dithionite (Roessler et al., 2003), as well as electrochemical vat dye reduction (Roessler and Jin, 2003). Moreover, it has been shown that the reductive decolorization of an anthraquinone dye by mesophilic anaerobic sludge can be accelerated by quinones (Bisschops et al., 2005; Dos Santos et al., 2005a). Both types of dyes are structures with quinone moieties, and their decolorization is presumably a result of the reduction of quinone to hydroquinone. Although this reaction can be assumed reversible once the dyes are again exposed to oxygen (Seignez et al., 1996), the results of studies to anaerobic treatment of anthraquinone dyes normally point at irreversible reduction (Fontenot et al., 2002; Dos Santos et al., 2005a).

\subsection{Reduction of soluble inorganic electron acceptors}

A small number of papers mention a role of RM in the reduction of soluble inorganic electron acceptors, i.e. 'normal' electron acceptors such as dissolved oxygen and nitrate, as well as less usual electron acceptors such as perchlorate and selenite. The reduction of some of these electron acceptors can be a purely chemical reaction (e.g. chemical sulfide oxidation by oxygen) but most electron acceptors exclusively require biotransformation for their reduction, coupled to the oxidation of primary electron donors. A crucial difference with the reductive biotransformation reactions dealt with so far is that the biological reduction of these soluble inorganic electron acceptors typically requires their uptake by the cells. As most external RM do not cross cell membranes, they are not likely to play a role in shuttling electrons directly to these electron acceptors. RM may only play a role in accelerating these reductions by transporting electrons to the cells. It has been established that many facultative anaerobes can capture electrons from chemically reduced AQDS ( $\left.\mathrm{AH}_{2} \mathrm{QDS}\right)$ to respire oxygen and nitrate, but not $\mathrm{Fe}(\mathrm{III}), \mathrm{Mn}(\mathrm{IV})$ and elemental sulfur (Coates et al., 2002). In this study, $\mathrm{AH}_{2} \mathrm{QDS}$ was not added as a mediator, but as a bulk electron donor in the absence of an intrinsic mechanism of AQDS reduction. In other studies, an (electro)chemical source of electrons was available, enabling RM to shuttle electron from this source to the cell. AQDS has been successfully applied in bioelectric perchlorate reduction to shuttle electrons from a cathode to the dissimilatory perchlorate reducing bacteria Dechloromonas and Azospira (Thrash et al., 2007). Likewise, it has been observed that 1,2-naphthoquinone4-sulfonate stimulates the simultaneous removal of sulfide and nitrate, possibly by facilitating the transfer electrons from sulfide to denitrifying bacteria (Aranda-Tamaura et al., 2007). In the absence of either an electrochemical source of electrons or a bulk reductant that reacts directly with the mediator, evidence for a role of RM is very limited. The observation that arsenate $(\mathrm{As}(\mathrm{V}))$ reduction by lactate-fed anaerobic granular sludge was somewhat accelerated by AQDS (Field et al., 2004) may have been related to AQDS reduction coupled to lactate oxidation followed by the use of $\mathrm{AH}_{2} \mathrm{QDS}$ as electron donor for arsenate-reducing bacteria. These results contrast the observation that AQDS had no effect on the reduction of arsenate by VFA-fed anaerobic sludge in continuous packed-column reactors (SierraAlvarez et al., 2005). Another study reported an accelerating effect of AQDS in combination with yeast extract on the reduction of selenite (Se(IV)) to insoluble selenium $\left(\mathrm{Se}^{0}\right)$ by glucose-fed Enterobacter taylorae. In contrast, AQDS did not affect the rate of the reduction of selenate ( $\mathrm{Se}(\mathrm{VI}))$ to $\mathrm{Se}(\mathrm{IV})$. The different results regarding the effect of AQDS on the two steps of Se(IV) reduction were attributed to redox potential differences between the couples involved (Zhang et al., 2007) but it may also have been due to the location of the reaction, with AQDS only able to interfere with Se(IV) reduction. It has been pointed out that, in order to avoid intracellular precipitation of 
insoluble products (in this case $\mathrm{Se}^{0}$ ), bacteria are more likely to use an exocellular respiratory mechanism (Gralnick and Newman, 2007).

\subsection{Reduction of insoluble materials (minerals and anodes)}

Microbial processes depending on the transfer of electrons to solid electron acceptors present a highly relevant category of oxidationreduction reactions with a significant role of RM. These involve the reduction of insoluble metal oxides, e.g. dissimilatory $\mathrm{Fe}(\mathrm{III})-$ and $\mathrm{Mn}$ (IV)-reduction, and anode reduction for electricity generation in microbial fuel cells (MFC). These processes have been the subject of a large number of research papers, and the mechanism of extracellular electron transfer, which is largely similar in both processes, has received ample attention and several review articles have been published, some of them mainly focused on respiration of minerals (Newman, 2001), others exclusively on MFC (Rabaey and Verstraete, 2005; Bullen et al., 2006; Logan et al., 2006; Lovley, 2006; Davis and Higson, 2007; Du et al., 2007; Schröder, 2007), others on both (Stams et al., 2006; Gralnick and Newman, 2007). For the transfer of electrons from the oxidation of primary substrates by bacteria to either oxidized metal minerals or the anode surface, one can distinguish between direct and mediated electron transfer (DET and MET, respectively). DET is based on membrane-bound enzymes and direct physical contact of the cell membrane or a membrane organelle (electropili) with the solid electron acceptor. In contrast, MET depends on RM, either externally added compounds such as AQS or shuttling compounds produced by the bacteria themselves, such as phenazines. In addition, in MFC an in-between category of electron transfer that has been alternately ranged under DET or MET, uses non-diffusive mediators immobilized on the anode surface. It is interesting to note the entanglement that may arise from the concepts: whereas the presence of RM is not an exclusive prerequisite for electricity generation, it may very well be that the mechanism of electron transfer in so-called 'mediator-less' MFCs depends (partly) on biogenic diffusive mediators. Moreover, stimulation of electricity production by non-diffusive mediators on the electrode surface still requires a DET or MET reduction mechanism.

Several metal-reducing bacteria, such as species of Geobacter, Shewanella and Rhodoferax, are capable of DET via c-type cytochromes, and it has recently been demonstrated that at least some of them (e.g. Geobacter sulfurreducens and S. putrefaciens) have the additional quality of forming electrically conductive pili or nanowires that enable them to reach electron-accepting surfaces at a certain distance from the cell (Reguera et al., 2005; Gorby et al., 2006). The other mechanism of electron transfer, 'self-mediated' MET via biogenic RM, is also receiving growing attention. It has been shown that Pseudomonas auruginosa produces pycocyanine and phenazine-1-carboxamide as RM (Rabaey et al., 2005). Moreover, recent evidence showed that several Shewanella species excrete the flavins riboflavin and FMN that serve as RM in electron transfer reaction (Marsili et al., 2008; Von Canstein et al., 2008). As several RM have lower redox potentials than the cytochromes responsible for DET, it has been pointed out that the amount of extractable electric energy can be higher with MET than with DET (Schröder, 2007). An important drawback of MET, however, is that diffusive mediators (either externally added or endogenously produced) may easily be lost from continuous flow systems such as MFC.

\subsection{Electron transfer from cathodes}

Whereas RM can be involved in transferring electrons to fuel cell anodes, they also can facilitate the transfer of electrons from fuel cell cathodes to terminal electron acceptors. Especially mediated electrochemical cathodic reduction of oxygen to superoxide and peroxide has been thoroughly investigated, as summarized in the review article by Šljukic et al. (2005). In addition, RM have been used to shuttle electrons from cathodes to bacteria, either for the reduction of electron accepting components such as TCE (Aulenta et al., 2007) perchlorate (Thrash et al., 2007) and sulfate (Kim et al., 1990), or for manipulating product formation of fermentative conversions such as glucose fermentation to propionate by a Propionibacterium species (Emde and Schink, 1990) or to butanol by Clostridium acetobutylicum (Peguin and Soucaille, 1996). In analogy to anodic reduction, cathodic oxidation can be based on MET as well as on DET. A recent review article on Bioelectrical Reactors (BER) provides an adequate summary of electrically stimulated biotransformation reactions and the mechanisms of electron transfer (Thrash and Coates, 2008).

\subsection{Sulfide oxidation}

While the redox couple sulfide/sulfur(polysulfide) is a potential electron shuttle in biotransformation reactions, e.g. reductive dehalogenation (De Wever et al., 2000), the oxidation of sulfide itself may be affected by external RM. It has been demonstrated that dissolved organic matter (DOM, peat humic acid) plays a redox-active role in sulfide cycling (Heitmann and Blodau, 2006; Blodau et al., 2007; Heitmann et al., 2007). It was also observed that the presence of DOM affects the way sulfide is oxidized, with thiosulfate rather than elemental sulfur or polysulfide as the main reaction products (Heitmann and Blodau, 2006; Heitmann et al., 2007). Furthermore, some chemical sulfide oxidation processes in practice, so-called 'liquid redox processes', use catalysts (Dalrymple et al., 1989). For example, in the Stretfort process, sulfide is oxidized by oxidized transition metals that are regenerated by organic RM that eventually shuttle electrons to oxygen (Steudel, 1996). Moreover, activated carbon, which is known to act as RM in azo dye reduction (Van der Zee et al., 2003; Mezohegyi et al., 2007) is also known to catalyze the reaction between sulfide and oxygen (Choi et al., 1991; Dalai et al., 1999). In this case, however, it is unknown whether an electron shuttling mechanism is involved or whether it is merely surface-catalysis.

\subsection{Enzymatic oxidation of organic compounds}

Several oxidative enzymes, especially laccases and peroxidases, show higher activity or depend on RM for the oxidation of wide array of organic substrates. Typical RM of these kind of enzymes are e.g. veratryl alcohol (3,4-dimethoxy benzyl alcohol) and 1-hydroxybenzotriazole, logically compounds with a higher $E_{0}{ }^{\prime}$ than the RM in reductive biotransformation reactions. A recent review paper on this topic provides a comprehensive summary (Husain and Husain, 2008).

\section{Future perspectives on the application of redox mediators on the reductive (bio)transformation of contaminants}

\subsection{Introduction}

There certainly are great potentials for the application of RM on the reductive (bio)transformation of several priority pollutants both in contaminated groundwater and in wastewaters originated from different industrial sectors. Nonetheless, to warrant a successful output of the application of RM, several important topics should be considered in future research. The purpose of this section is to underline the main research areas demanded to enhance the applicability of RM in aquifer restoration and wastewater treatment.

\subsection{Cost-effective sources of redox active groups}

Several redox active groups have been reported to play a significant role in the reductive (bio)transformation of priority pollutants. The large list of redox active molecules includes cytochromes (Picardal et al., 1993), flavines (Guerrero-Barajas and Field, 2005), cobalamins (Zou et al., 2000), porphyrins (Koons et al., 2001), pyridines (Lee et al., 1999), phenazines (Hernandez et al., 2004) and quinones (Field and Cervantes, 2005). 
Quinones, active functional groups in humus, have been proposed as the most appropriate RM for the reductive (bio)transformation of a number of priority pollutants (Field and Cervantes, 2005). The main reason for considering quinones for redox (bio)transformation processes is that they are very abundant in humus, the most plentiful and cheaply available organic source in the biosphere. Moreover, the remarkable stability of HS in the environment, evidenced by their large residence time (>500 years) (Stevenson, 1994), prevents the deterioration of this material during bioremediation processes. Furthermore, humus is generally considered as a non-hazardous material and does not lead to the production of toxic byproducts (Perminova et al., 2005).

However, to take advantage of this abundant source of RM, careful selection of the origin of the humic material should be undertaken. Certainly, the ECC of several HS from a variety of origins was quantified (Ratasuk and Nanny, 2007). Highly aliphatic FA and HA extracted from different landfill leachates did not have any ECC and therefore are not recommended as a suitable source for RM. In contrast, FA and HA originated from peat, river sediments or soils showed an ECC ranging from 25 up to 538 micro-equivalents per $g \mathrm{HS}$ at different $\mathrm{pH}$ values.

\subsection{Engineered HS for their application as RM in redox reactions}

As underlined by Perminova et al. (2005), the principal factor limiting the application of HS in remediation technologies is the intrinsic variability in redox properties observed among humic materials and their fractions. Therefore, engineered humic materials are demanded in order to accomplish the redox mediating properties required during the conversion of a large number of priority pollutants through redox reactions.

Quinoid-enriched humic materials with enhanced redox properties were synthesized by two different strategies (Perminova et al., 2005). The first approach was related to the oxidation of phenolic fractions associated with the humic aromatic core. In a second strategy, polycondensation of these phenolic fragments was carried out with hydroquinone and catechol. Redox characterization of the copolymers obtained revealed that the reducing capacity of this synthesized humic material ranged between 1 and $4 \mathrm{mmol} / \mathrm{g}$, which was much higher than the parent materials and the oxidized derivatives. Therefore, preferential application of the copolycondensation approach was advised.

Water soluble sulfonated humic materials were produced from leonardite and peat in order to evaluate their metal-binding capacity for $\mathrm{Fe}(\mathrm{III})$ and $\mathrm{Hg}$ (II) (Yudov et al., 2005). Enrichment of HA with sulfonate groups was postulated as an approach for remediation techniques applied during heavy metals removal from contaminated sites. The solubility of metal-HA complexes was estimated as an amount of strong metal-binding sites (SBS) in the humic materials. The sulfonated materials were characterized by a 2-fold increase in the amount of SBS compared to the parent materials. The enhanced solubility achieved in HA by this strategy may also be useful to distribute redox active groups of HA in contaminated aquifers for restoration purposes.

Different humic derivatives have also been synthesized and immobilized in silica gel to enhance their applicability in remediation technologies based on reactive barriers (Perminova et al., 2007). Nevertheless, considering the wide variety of redox reactions in which HS could be demanded, further research is required in order to obtain engineered HS, which fulfill different redox characteristics.

\subsection{Immobilization of $R M$}

Quinoid RM not necessarily have to be supplied abundantly in bioremediation processes to accelerate the redox transformation of different contaminants, as they are being recycled during the transfer of electron from an electron donor to the terminal electron acceptor (Fig. 1). However, continuous addition of RM should be supplied in anaerobic bioreactors in order to achieve increased conversion rates, which raise the costs of treatment.

An approach to eliminate the prerequisite of continuous supply of $\mathrm{RM}$ is to create a niche for their immobilization in anaerobic bioreactors. However, scarce attempts to apply immobilized quinoid RM for the anaerobic conversion of priority pollutants have been reported. One of the alternatives available considers the application of activated carbon (AC) as a natural source of quinoid RM and its potential for reducing azo dyes have been explored in anaerobic bioreactors (Van der Zee et al., 2003). An AC-amended bioreactor performed a higher decolorizing capacity ( $>90 \%$ of decolorization) compared to a bioreactor control lacking AC ( $<40 \%$ of decolorization) during the reductive decolorization of RR2. Evidence indicated that the redox mediating capacity of $\mathrm{AC}$ was the main cause of the enhanced decolorization observed as the improved RR2 removal observed was 121-fold higher than the adsorption capacity of the AC. However, the catalytic effects of AC gradually decrease attributed to its continuing wash-out from the reactor. More recently, an upflow packed-bed reactor (UPBR) containing biological AC was tested for the continuous decolorization of AO7 (Mezohegyi et al., 2007). The treatment system achieved high decolorization efficiency of AO7 at very short space times: up to $99 \%$ decolorization in $2 \mathrm{~min}$. Thus, the UPBR seems to be a promising system for anaerobic redox conversions, but needs to be proved with more recalcitrant pollutants.

Another immobilizing approach reported is to insert quinoid RM within different materials through polymerization procedures (Guo et al., 2007). Through this immobilizing technique, quinoid RM remain entrapped within the synthesized polymer keeping their redox mediating capacity. In this study, anthraquinone (AQ) entrapped in calcium alginate promoted up to 2-fold increase on the rate of decolorization of different azo dyes by a salt-tolerant bacterial culture, compared to controls lacking AQ. Disadvantages of this strategy are: 1) mass transfer limitations since a major part of the RM is embedded within the polymeric material making its accessibility dependent on diffusion; 2) gradual lost of redox catalysts due to disruption of the polymeric material owing to weak mechanical strength of the materials explored.

Lately, functionalized polypyrrole (PPy) composites were enriched with AQDS during the electropolymerization of pyrrole monomer on activated carbon felt (ACF) electrode. The resulting composite (ACF/ PPy/AQDS) was tested as a RM for the reductive biotransformation of nitroaromatic compounds (Li et al., 2008). This study showed that ACF/PPy/AQDS exhibited good catalytic activity and stability and its addition effectively accelerated the reductive transformation of different nitroaromatic pollutants by an anaerobic consortium. Thus, this strategy represents a promising tool to immobilize RM for remediation technologies.

\subsection{Overcoming limitations during catalysis involving RM}

As discussed in the present review, the catalytic effects of RM greatly fluctuate among the different redox reactions studied so far. On one hand, the literature provides plentiful information documenting the enhancement of redox reactions involved during the (bio) transformation of several organic and inorganic pollutants in which the rate of conversion increased several orders of magnitude. On the other hand, several studies evidenced inhibitory effects of HS during the reductive (bio)transformation of electron-withdrawing contaminants. For instance, abiotic incubations amended with different concentrations of HA showed a decrease of up to $50 \%$ on the reduction rate of NB, TCE and CT by ZVI, compared to controls incubated in the absence of HA (Tratnyek et al., 2001). The inhibitory effects of HA were attributed to competitive sorption of HA onto reactive sites of ZVI limiting the transfer of electrons between this electron donor and the 
electron-withdrawing pollutants. The last scenario was also observed during the reductive transformation of PCE (Doong and Lai, 2005) and 2,6-DNAH (Keum and Li, 2004) by ZVI in which HA amendments caused a decrease of up to 10 -fold on the rate of reduction of these contaminants compared to controls lacking HA.

Not only HA, but also model quinoid RM, such as AQDS, have also inhibited the reductive conversion of electron-withdrawing contaminants. Indeed, the microbial dechlorination of 2,3,5,6-tetrachlorobiphenyl by a bacterial enrichment culture was completely inhibited by the addition of HA or AQDS by non-clarified mechanisms (Cutter et al., 1998). Furthermore, AQDS addition to a methanogenic enrichment culture promoted a higher reduction rate of $\mathrm{FeOOH}$, but decreased both the rate of dechlorination of pentachloroaniline and methanogenesis probably due to competition among these processes for the reducing equivalents available in the incubations (Okutman Tas and Pavlostathis, 2007).

The information available in the literature provides a broad perspective of the different experimental conditions $(\mathrm{pH}$, temperature, type and concentration of RM, type and concentration of electron donor, and redox potential), which affect the catalytic impact of RM. However, scarce information has been reported to elucidate the mechanisms by which RM are involved in different redox reactions and the bottlenecks to consider during redox catalysis. In some cases, the reduction of RM by different microorganisms has been pointed out as the rate-limiting step during redox reactions (Rau et al., 2002). In contrast, other studies evidenced that the reduction of oxidized contaminants by reduced RM is the rate-limiting step (Rau et al., 2002; Jiang and Kappler, 2008). Therefore, further research is demanded in order to elucidate the mechanisms limiting the catalytic effects of RM during the anaerobic (bio)transformation of priority pollutants.

\section{Acknowledgements}

F.P. van der Zee thanks the Portuguese Fundação para a Ciência e a Tecnologia for financial support (Grant SFRH/BPD/39086/2007). F. J. Cervantes greatly acknowledges a grant from Council of Science and Technology of Mexico (Grant SEP-CONACYT-C02-55045).

\section{References}

Albuquerque MGE, Lopes AT, Serralheiro ML, Novais JM, Pinheiro HM. Biological sulphate reduction and redox mediator effects on azo dye decolourisation in anaerobicaerobic sequencing batch reactors. Enzyme Microb Technol 2005;36:790-9.

Angelova B, Avramova T, Stefanova L, Mutafov S. Temperature effect on bacterial azo bond reduction kinetics: an Arrhenius plot analysis. Biodegradation 2008;19:387-93.

Aranda-Tamaura C, Estrada-Alvarado MI, Texier AC, Cuervo F, Gomez J, Cervantes FJ Effects of different quinoid redox mediators on the removal of sulphide and nitrate via denitrification. Chemosphere 2007;69:1722-7.

Aulenta F, Catervi A, Majone M, Panero S, Reale P, Rossetti S. Electron transfer from a solid-state electrode assisted by methyl viologen sustains efficient microbial reductive dechlorination of TCE. Environ Sci Technol 2007;41:2554-9.

Barkovskii AL, Adriaens P. Impact of humic constituents on microbial dechlorination of polychlorinated dioxins. Environ Toxicol Chem 1998;17:1013-20.

Bechtold T, Gutmann R, Burtscher E, Bobleter O. Cyclic voltammetric study of anthraquinone-2-sulfonate in the presence of Acid Yellow 9. Electrochim Acta 1997;42:3483-7.

Bhushan B, Halasz A, Hawari J. Effect of iron(III), humic acids and anthraquinone-2,6disulfonate on biodegradation of cyclic nitramines by Clostridium sp. EDB2. J Appl Microbiol 2006;100:555-63.

Bisschops I, Dos Santos AB, Spanjers $H$. Waste sizing solution as co-substrate fo anaerobic decolourisation of textile dyeing wastewaters. Water Sci Technol 2005;52:397-403.

Blodau C, Mayer B, Peiffer S, Moore TR. Support for an anaerobic sulfur cycle in two Canadian peatland soils. J Geophys Res 2007;112. doi:10.1029/2006JG000364.

Borch T, Inskeep WP, Harwood JA, Gerlach R. Impact of ferrihydrite and anthraquinone2,6-disulfonate on the reductive transformation of 2,4,6-trinitrotoluene by a Grampositive fermenting bacterium. Environ Sci Technol 2005;39:7126-33.

Bradley PM, Chapelle FH, Lovley DR. Humic acids as electron acceptors for anaerobic microbial oxidation of vinyl chloride and dichloroethene. Appl Environ Microbio 1998;64:3102-5.

Bragger JL, Lloyd AW, Soozandehfar SH, Bloomfield SF, Marriott C, Martin GP Investigations into the azo reducing activity of a common colonic microorganism. Int J Pharm 1997;157:61-71.

Brown JP. Reduction of polymeric azo and nitro dyes by intestinal bacteria. Appl Environ Microbiol 1981;41:1283-6.
Bullen RA, Arnot TC, Lakeman JB, Walsh FC. Biofuel cells and their development. Biosens Bioelectron 2006;21:2015-45.

Cervantes FJ, Van der Velde S, Lettinga G, Field JA. Competition between methanogenesis and quinone respiration for ecologically important substrates in anaerobic consortia. FEMS Microbiol Ecol 2000a;34:161-71.

Cervantes FJ, Van der Velde S, Lettinga G, Field JA. Quinones as terminal electron acceptors for anaerobic microbial oxidation of phenolic compounds. Biodegradation 2000b;11:313-21.

Cervantes FJ, Dijksma W, Duong-Dac T, Ivanova A, Lettinga G, Field JA. Anaerobic mineralization of toluene by enriched sediments with quinones and humus as terminal electron acceptors. Appl Environ Microbiol 2001a;67:4471-8.

Cervantes FJ, Van der Zee FP, Lettinga G, Field JA. Enhanced decolourisation of Acid Orange 7 in a continuous UASB reactor with quinones as redox mediators. Water Sci Technol 2001b;44:123-8.

Cervantes FJ, Vu-Thi-Thu L, Lettinga G, Field JA. Quinone-respiration improves dechlorination of carbon tetrachloride by anaerobic sludge. Appl Microbiol Biotechnol 2004;64:702-11.

Cervantes FJ, Enriquez JE, Mendoza-Hernandez MR, Razo-Flores E, Field JA. The role of sulphate reduction on the reductive decolorization of the azo dye reactive orange 14. Water Sci Technol 2006;54:171-7.

Chang JS, Kuo TS. Kinetics of bacterial decolorization of azo dye with Escherichia coli NO3. Biores Technol 2000;75:107-11.

Chang JS, Chou C, Lin YC, Lin PJ, Ho JY, Hu TL. Kinetic characteristics of bacterial azo-dye decolorization by Pseudomonas luteola. Water Res 2001;35:2841-50.

Choi JJ, Hirai M, Shoda M. Catalytic oxidation of hydrogen sulfide by air over an activated carbon filter. Appl Catal A-Gen 1991;79:241-8.

Christiansen N, Christensen SR, Arvin E, Ahring BK. Transformation of tetrachloroethene in an upflow anaerobic sludgeblanket reactor. Appl Microbiol Biotechnol 1997;47:91-4.

Chung KT, Fulk GE, Egan M. Reduction of azo dyes by intestinal anaerobes. Appl Environ Microbiol 1978;35:558-62.

Coates JD, Cole KA, Chakraborty R, O'Connor SM, Achenbach LA. Diversity and ubiquity of bacteria capable of utilizing humic substances as electron donors for anaerobic respiration. Appl Environ Microbiol 2002;68:2445-52.

Cohen B. The bacterial culture as an electrical half-cell. J Bacteriol 1931;21:18-9.

Collins R, Picardal F. Enhanced anaerobic transformations of carbon tetrachloride by soil organic matter. Environ Toxicol Chem 1999;18:2703-10.

Curtis GP, Reinhard M. Reductive dehalogenation of hexachloroethane, carbon tetrachloride, and bromoform by anthraquinone disulfonate and humic acid. Environ Sci Technol 1994:28:2393-401.

Cutter L, Sowers KR, May HD. Microbial dechlorination of 2,3,5,6-tetrachlorobiphenyl under anaerobic conditions in the absence of soil or sediment. Appl Environ Microbiol 1998;64:2966-9.

Dalai AK, Majumdar A, Tollefson EL. Low temperature catalytic oxidation of hydrogen sulfide in sour produced wastewater using activated carbon catalysts. Environ Sci Technol 1999;33:2241-6.

Dalrymple DA, Trofe TW, Evans JM. Liquid redox sulfur recovery options, costs, and environmental considerations. Environ Prog 1989;8:217-22.

Davis F, Higson SPJ. Biofuel cells-recent advances and applications. Biosens Bioelectron 2007;22:1224-35.

De Wever H, Cole JR, Fettig MR, Hogan DA, Tiedje JM. Reductive dehalogenation of trichloroacetic acid by Trichlorobacter thiogenes gen. nov., sp. nov. Appl Environ Microbiol 2000;66:2297-301.

Donlon BA, Razo-Flores E, Lettinga G, Field JA. Continuous detoxification, transformation and degradation of nitrophenols in upflow anaerobic sludge blanket (UASB) reactors. Biotechnol Bioeng 1996;51:439-49.

Doong R-A, Chiang H-C. Transformation of carbon tetrachloride by thiol reductants in the presence of quinone compounds. Environ Sci Technol 2005;39:7460-8.

Doong R-A, Lai Y-J. Dechlorination of tetrachloroethylene by palladized iron in the presence of humic acid. Water Res 2005:39:2309-18.

Dos Santos AB, Cervantes FJ, Yaya-Beas RE, Van Lier JB. Effect of redox mediator, AQDS, on the decolourisation of a reactive azo dye containing triazine group in a thermophilic anaerobic EGSB reactor. Enzyme Microb Technol 2003;33:942-51.

Dos Santos AB, Bisschops IAE, Cervantes FJ, Van Lier JB. Effect of different redox mediators during thermophilic azo dye reduction by anaerobic granular sludge and comparative study between mesophilic $\left(30^{\circ} \mathrm{C}\right)$ and thermophilic $\left(55^{\circ} \mathrm{C}\right)$ treatments for decolourisation of textile wastewaters. Chemosphere 2004a;55:1149-57.

Dos Santos AB, Cervantes FJ, Van Lier JB. Azo dye reduction by thermophilic anaerobic granular sludge, and the impact of the redox mediator anthraquinone-2,6-disulfonate (AQDS) on the reductive biochemical transformation. Appl Microbiol Biotechnol 2004b:64:62-9.

Dos Santos AB, Bisschops IAE, Cervantes FJ, Van Lier JB. The transformation and toxicity of anthraquinone dyes during thermophilic $\left(55^{\circ} \mathrm{C}\right)$ and mesophilic $\left(30^{\circ} \mathrm{C}\right)$ anaerobic treatments. J Biotechnol 2005a;115:345-53.

Dos Santos AB, De Madrid MP, Stams AJM, Van Lier JB, Cervantes FJ. Azo dye reduction by mesophilic and thermophilic anaerobic consortia. Biotechnol Prog 2005b;21:1140-5.

Dos Santos AB, Traverse J, Cervantes FJ, Van Lier JB. Enhancing the electron transfer capacity and subsequent color removal in bioreactors by applying thermophilic anaerobic treatment and redox mediators. Biotechnol Bioeng 2005c;89:42-52.

Dos Santos AB, Traverse J, Cervantes FJ, Van Lier JB. Thermophilic treatment by anaerobic granular sludge as an effective approach to accelerate the electron transfer and improve the reductive decolorization of azo dyes in bioreactors. Water Sci Technol 2005d;52:363-9.

Dos Santos AB, De Madrid MP, De Bok FAM, Stams AJM, Van Lier JB, Cervantes FJ. The contribution of fermentative bacteria and methanogenic archaea to azo dye reduction by a thermophilic anaerobic consortium. Enzyme Microb Technol 2006;39:38-46. 
Dror I, Schlautman MA. Cosolvent effect on the catalytic reductive dechlorination of PCE. Chemosphere 2004;57:1505-14.

Du Z, Li H, Gu T. A state of the art review on microbial fuel cells: a promising technology for wastewater treatment and bioenergy. Biotechnol Adv 2007;25:464-82.

Dubin P, Wright KL. Reduction of azo food dyes in cultures of Proteus vulgaris. Xenobiotica 1975;5:563-671.

Dunnivant FM, Schwarzenbach RP, Macalady DL. Reduction of substituted nitrobenzenes in aqueous solutions containing natural organic matter. Environ Sci Technol 1992;26:2133-41.

Emde R, Schink B. Enhanced propionate formation by Propionibacterium freudenreichii subsp. freudenreichii in a three-electrode amperometric culture system. Appl Environ Microbiol 1990;56:2771-6.

Encinas-Yocupicio AA, Razo-Flores E, Sanchez-Diaz F, Dos Santos AB, Field JA, Cervantes FJ. Catalytic effects of different redox mediators on the reductive decolorization of azo dyes. Water Sci Technol 2006:54:165-70.

Field JA, Brady J. Riboflavin as a redox mediator accelerating the reduction of the azo dye Mordant Yellow 10 by anaerobic granular sludge. Water Sci Technol 2003;48:187-93.

Field JA, Cervantes FJ. Microbial redox reactions mediated by humus and structurally related quinones. In: Perminova IV, Hatfield K, Hertkorn N, editors. Use of humic substances to remediate polluted environments: from theory to practice, vol. 52. Dordrecht, The Netherlands: Springer; 2005. p. 343-52.

Field JA, Stams AJM, Kato M, Schraa G. Enhanced biodegradation of aromatic pollutants in cocultures of anaerobic and aerobic bacterial consortia. Anton Leeuw Int J G 1995;67:47-77.

Field JA, Sierra-Alvarez R, Cortinas I, Feijoo G, Moreira M, Kopplin M, et al. Facile reduction of arsenate in methanogenic sludge. Biodegradation 2004;15:185-96.

Fontenot EJ, Beydilli MI, Lee YH, Pavlostathis SG. Kinetics and inhibition during the decolorization of reactive anthraquinone dyes under methanogenic conditions. Water Sci Technol 2002;45:215-20.

Fredrickson JK, Kostandarithes HM, Li SW, Plymale AE, Daly MJ. Reduction of Fe(III), Cr (VI), U(VI), and $\mathrm{Tc}(\mathrm{VII})$ by Deinococcus radiodurans R1. Appl Environ Microbiol 2000;66:2006-11.

Gorby YA, Yanina S, McLean JS, Rosso KM, Moyles D, Dohnalkova A, et al. Electrically conductive bacterial nanowires produced by Shewanella oneidensis strain MR-1 and other microorganisms. Proc Natl Acad Sci USA 2006;103:11358-63.

Gralnick JA, Newman DK. Extracellular respiration. Mol Microbiol 2007;65:1-11.

Guerrero-Barajas C, Field JA. Riboflavin- and cobalamin-mediated biodegradation of chloroform in a methanogenic consortium. Biotechnol Bioeng 2005;89:539-50.

Guo J, Zhou J, Wang D, Tian C, Wang P, Salah Uddin M, et al. Biocalalyst effects of immobilized anthraquinone on the anaerobic reduction of azo dyes by the salttolerant bacteria. Water Res 2007;41:426-32.

Guo J, Zhou J, Wang D, Yang J, Li Z. The new incorporation bio-treatment technology of bromoamine acid and azo dyes wastewaters under high-salt conditions. Biodegradation 2008;19:93-8.

Hakala JA, Chin Y-P, Weber EJ. Influence of dissolved organic matter and Fe(II) on the abiotic reduction of pentachloronitrobenzene. Environ Sci Technol 2007;41:7337-42.

Hashsham SA, Freedman DL. Enhanced biotransformation of carbon tetrachloride by Acetobacterium woodii upon addition of hydroxocobalamin and fructose. Appl Environ Microbiol 1999;65:4537-42.

Heitmann T, Blodau C. Oxidation and incorporation of hydrogen sulfide by dissolved organic matter. Chem Geol 2006;235:12-20.

Heitmann T, Goldhammer T, Beer J, Blodau C. Electron transfer of dissolved organic matter and its potential significance for anaerobic respiration in a northern bog. Glob Change Biol 2007;13:1771-85.

Hernandez ME, Kappler A, Newman DK. Phenazines and other redox-active antibiotics promote microbial mineral reduction. Appl Environ Microbiol 2004;70:921-8.

Hofstetter TB, Heijman CG, Haderlein SB, Holliger C, Schwarzenbach RP. Complete reduction of TNT and other (poly)nitroaromatic compounds under iron-reducing subsurface conditions. Environ Sci Technol 1999;33:1479-87.

Hong YG, Gu J, Xu ZC, Xu MY, Sun GP. Humic substances act as electron acceptor and redox mediator for microbial dissimilatory azoreduction by Shewanella decolorationis S12. J Microbiol Biotechnol 2007;17:428-37.

Husain M, Husain Q. Applications of redox mediators in the treatment of organic pollutants by using oxidoreductive enzymes: a review. Crit Rev Environ Sci Technol 2008;38:1-42.

Jiang J, Kappler A. Kinetics of microbial and chemical reduction of humic substances: implications for electron shuttling. Environ Sci Technol 2008;42:3563-9.

Kappler A, Haderlein SB. Natural organic matter as reductant for chlorinated aliphatic pollutants. Environ Sci Technol 2003;37:2714-9.

Keck A, Klein J, Kudlich M, Stolz A, Knackmuss HJ, Mattes R. Reduction of azo dyes by redox mediators originating in the naphthalenesulfonic acid degradation pathway of Sphingomonas sp. strain BN6. Appl Environ Microbiol 1997;63:3684-90.

Keck A, Rau J, Reemtsma T, Mattes R, Stolz A, Klein J. Identification of quinoide redox mediators that are formed during the degradation of naphthalene-2-sulfonate by Sphingomonas xenophaga BN6. Appl Environ Microbiol 2002;68:4341-9.

Keum YS, Li QX. Reduction of nitroaromatic pesticides with zero-valent iron. Chemosphere 2004;54:255-63.

Kim TS, Kim HY, Kim BH. Petroleum desulfurization by Desulfovibrio desulfuricans M6 using electrochemically supplied reducing equivalent. Biotechnol Lett 1990;12:757-60.

Koons BW, Baeseman JL, Novak PJ. Investigation of cell exudates active in carbon tetrachloride and chloroform degradation. Biotechnol Bioeng 2001;74:12-7.

Kudlich M, Keck A, Klein J, Stolz A. Localization of the enzyme system involves in anaerobic reduction of azo dyes by Sphingomonas sp. strain BN6 and effect of artificial redox mediators on the rate of azo dye reduction. Appl Environ Microbiol 1997;63:3691-4.

Kwon MJ, Finneran KT. Microbially mediated biodegradation of hexahydro-1,3,5trinitro-1,3,5- triazine by extracellular electron shuttling compounds. Appl Environ Microbiol 2006;72:5933-41.
Kwon M, Finneran K. Biotransformation products and mineralization potential for hexahydro-1,3,5-trinitro-1,3,5-triazine (RDX) in abiotic versus biological degradation pathways with anthraquinone-2,6-disulfonate (AQDS) and Geobacter metallireducens. Biodegradation 2008;19:705-15.

Lee C-H, Lewis TA, Paszczynski A, Crawford RL. Identification of an extracellular catalyst of carbon tetrachloride dehalogenation from Pseudomonas stutzeri strain KC as pyridine-2,6-bis(thiocarboxylate). Biochem Biophys Res Commun 1999;261:562-6.

Li L, Wang J, Zhou J, Yang F, Jin C, Qu Y, et al. Enhancement of nitroaromatic compounds anaerobic biotransformation using a novel immobilized redox mediator prepared by electropolymerization. Biores Technol 2008;99:6908-16.

Logan BE, Hamelers B, Rozendal R, Schroder U, Keller J, Freguia S, et al. Microbial fue cells: methodology and technology. Environ Sci Technol 2006;40:5181-92.

Lovley DR. Bug juice: harvesting electricity with microorganisms. Nat Rev Mircobio 2006;4:497-508.

Lovley DR, Coates JD, Blunt-Harris EL, Phillips EJP, Woodward JC. Humic substances as electron acceptors for microbial respiration. Nature 1996;382:445-8.

Lovley DR, Fraga JL, Coates JD, Blunt Harris EL. Humics as an electron donor for anaerobic respiration. Environ Microbiol 1999;1:89-98.

Marsili E, Baron DB, Shikhare ID, Coursolle D, Gralnick JA, Bond DR. Shewanella secretes flavin that mediate extracellular electron transfer. Proc Natl Acad Sci USA 2008;105:3968-73.

Mezohegyi G, Kolodkin A, Castro UI, Bengoa C, Stuber F, Font J, et al. Effective anaerobic decolorization of azo dye Acid Orange 7 in continuous upflow packed-bed reacto using biological activated carbon system. Ind Eng Chem Res 2007;46:6788-92.

Newman DK. Microbiology - how bacteria respire minerals. Science 2001;292:1312-3.

Okutman Tas D, Pavlostathis SG. The influence of iron reduction on the reductive biotransformation of pentachloronitrobenzene. Eur J Soil Biol 2007;43:264-75.

Pandey A, Singh P, Iyengar L. Bacterial decolorization and degradation of azo dyes. Int J Biodeterioration Biodegradation 2007;59:73-84.

Pearce CI, Christie R, Boothman C, Von Canstein H, Guthrie JT, Lloyd JR. Reactive azo dye reduction by Shewanella strain J18 143. Biotechnol Bioeng 2006;95:692-703.

Pearce CI, Guthrie JT, Lloyd JR. Reduction of pigment dispersions by Shewanella strain J18 143. Dyes Pigm 2008;76:696-705.

Peguin S, Soucaille P. Modulation of metabolism of Clostridium acetobutylicum grown in chemostat culture in a three-electrode potentiostatic system with methyl viologen as electron carrier. Biotechnol Bioeng 1996;51:342-8.

Peijnenburg WJGM, 't Hart MJ, Den Hollander HA, Van de Meent D, Verboom HH, et al QSARs for predicting reductive transformation rate constants of halogenated aromatic hydrocarbons in anoxic sediment systems. Environ Toxicol Chem 1992;11:301-14.

Perlinger JA, Angst W, Schwarzenbach RP. Kinetics of the reduction of hexachloroethane by juglone in solutions containing hydrogen sulfide. Environ Sci Technol 1996;30:3408-17.

Perlinger JA, Buschmann J, Angst W, Schwarzenbach RP. Iron porphyrin and mercaptojuglone mediated reduction of polyhalogenated methanes and ethanes in homogeneous aqueous solution. Environ Sci Technol 1998;32:2431-7.

Perlinger JA, Kalluri VM, Venkatapathy R, Angst W. Addition of hydrogen sulfide to juglone. Environ Sci Technol 2002;36:2663-9.

Perminova IV, Kovalenko AN, Schmitt-Kopplin P, Hatfield K, Hertkorn N, Belyaeva EY, et al. Design of quinonoid-enriched humic materials with enhanced redox properties. Environ Sci Technol 2005;39:8518-24.

Perminova IV, Ponomarenko SA, Karpiouk LA, Hatfield K. Humic derivatives methods of preparation and use. PCT world patent, 2007; WO/2007/102750.

Picardal FW, Arnold RG, Couch H, Little AM, Smith ME. Involvement of cytochromes in the anaerobic biotransformation of tetrachloromethane by Shewanella putrefaciens 200. Appl Environ Microbiol 1993;59:3763-70.

Rabaey K, Verstraete W. Microbial fuel cells: novel biotechnology for energy generation. Trends Biotechnol 2005;23:291-8.

Rabaey K, Boon N, Höfte M, Verstraete W. Microbial phenazine production enhances electron transfer in biofuel cells. Environ Sci Technol 2005;39:3401-8.

Ramalho PA, Cardoso MH, Cavaco-Paulo A, Ramalho MT. Characterization of azo reduction activity in a novel ascomycete yeast strain. Appl Environ Microbiol 2004:70:2279-88.

Ratasuk N, Nanny MA. Characterization and quantification of reversible redox sites in humic substances. Environ Sci Technol 2007;41:7844-50.

Rau J. Stolz A. Oxygen-sensitive nitroreductases NfsA and NsfB of Escherichia coli function under anaerobic conditions as lawsone-dependent azo reductases. Appl Environ Microbiol 2003;69:3448-55

Rau J, Knackmuss HJ, Stolz A. Effects of different quinoid redox mediators on the anaerobic reduction of azo dyes by bacteria. Environ Sci Technol 2002;36:1497-504.

Razo-Flores E, Lettinga G, Field JA. Biotransformation and biodegradation of selected nitroaromatics under anaerobic conditions. Biotechnol Prog 1999;15:358-65.

Razo-Flores E, Macarie H, Morier F. Application of biological treatment systems for chemical and petrochemical wastewaters. In: Cervantes FJ, Pavlostathis SG, Van Haandel AC, editors. Advanced biological treatment processes for industrial wastewaters: principles \& applications. London, UK: IWA Publishing; 2006. p. 267-97.

Reguera G, McCarthy KD, Mehta T, Nicoll JS, Tuominen MT, Lovley DR. Extracellular electron transfer via microbial nanowires. Nature 2005;435:1098-101.

Rodgers JD, Bunce NJ. Treatment methods for the remediation of nitroaromatic explosives. Water Res 2001;35:2101-11.

Roessler A, Jin X. State of the art technologies and new electrochemical methods for the reduction of vat dyes. Dyes Pigm 2003;59:223-35

Roessler A, Crettenand D, Dossenbach O, Rys P. Electrochemical reduction of indigo in fixed and fluidized beds of graphite granules. J Appl Electrochem 2003;33:901-8.

Roxon JJ, Ryan AJ, Wright SE. Enzymatic reduction of tartrazine by Proteus vulgaris from rats. Food Cosmet Toxicol 1967;5:645-56. 
Russ R, Rau J, Stolz A. The function of cytoplasmic flavin reductases in the reduction of azo dyes by bacteria. Appl Environ Microbiol 2000;66:1429-34.

Schröder U. Anodic electron transfer mechanisms in microbial fuel cells and their energy efficiency. Phys Chem Chem Phys 2007;9:2619-29.

Scheline RR, Nygaard RT. Enzymatic reduction of the azo dye, Acid Yellow, by extracts of Streptococcus faecalis isolated from rat intestine. Food Cosmet Toxicol 1970;8:55-8.

Schwarzenbach RP, Stierli R, Lanz K, Zeyer J. Quinone and iron porphyrin mediated reduction of nitroaromatic compounds in homogeneous aqeous solution. Environ Sci Technol 1990;24:1566-74.

Scott DT, McKnight DM, Blunt Harris EL, Kolesar SE, Lovley DR. Quinone moieties act as electron acceptors in the reduction of humic substances by humics-reducing microorganisms. Environ Sci Technol 1998;32:2984-9.

Seignez C, Adler N, Suard JC, Peringer P. Aerobic and anaerobic biodegradability of 1anthraquinone sulphonate. Appl Microbiol Biotechnol 1996;45:719-22.

Semdé R, Pierre D, Geuskens G, Devleeschouwer M, Moes AJ. Study of some important factors involved in azo derivative reduction by Clostridium perfringens. Int J Pharm 1998;161:45-54.

Sierra-Alvarez R, Field JA, Cortinas I, Feijoo G, Moreira MT, Kopplin M, et al. Anaerobic microbial mobilization and biotransformation of arsenate adsorbed onto activated alumina. Water Res 2005;39:199-209.

Šljukic B, Banks CE, Compton RG. An overview of the electrochemical reduction of oxygen at carbon-based modified electrodes. J Iran Chem Soc 2005;2:1-25.

Stams AJM, De Bok FAM, Plugge CM, Van Eekert MHA, Dolfing J, Schraa G. Exocellular electron transfer in anaerobic microbial communities. Environ. Microbiol 2006;8:371-82.

Steudel R. Mechanism for the formation of elemental sulfur from aqueous sulfide in chemical and microbiological desulfurization processes. Ind Eng Chem Res 1996;35:1417-23.

Stevenson FJ. Humus chemistry: genesis, composition, reactions. New York: John Wiley and Sons, Inc.; 1994.

Stolz A. Basic and applied aspects in the microbial degradation of azo dyes. App Microbiol Biotechnol 2001;56:69-80.

Storck WJ, Tullo AH, Short PL, Tremblay JF. World chemical outlook. Chem Eng News 2005;83:15-29.

Thrash JC, Coates JD. Review: direct and indirect electrical stimulation of microbial metabolism. Environ Sci Technol 2008;42:3921-31.

Thrash JC, Van Trump JI, Weber KA, Miller E, Achenbach LA, Coates JD. Electrochemica stimulation of microbial perchlorate reduction. Environ Sci Technol 2007;41:1740-6.

Tratnyek PG, Macalady DL. Abiotic reduction of nitro aromatic pesticides in anaerobic laboratory systems. J Agric Food Chem 1989;37:248-54.
Tratnyek PG, Scherer MM, Deng BL, Hu SD. Effects of natural organic matter, anthropogenic surfactants, and model quinones on the reduction of contaminants by zero-valent iron. Water Res 2001;35:4435-43.

Van der Zee FP, Lettinga G, Field JA. The role of (auto)catalysis in the mechanism of anaerobic azo reduction. Water Sci Technol 2000;42:301-8.

Van der Zee FP, Bouwman RHM, Strik DPBTB, Lettinga G, Field JA. Application of redox mediators to accelerate the transformation of reactive azo dyes in anaerobic bioreactors. Biotechnol Bioeng 2001;75:691-701.

Van der Zee FP, Bisschops IAE, Lettinga G, Field JA. Activated carbon as an electron acceptor and redox mediator during the anaerobic biotransformation of azo dyes. Environ Sci Technol 2003;37:402-8.

Van der Zee FP, Villaverde S. Combined anaerobic-aerobic treatment of azo dyes-a short review of bioreactor studies. Water Res 2005;39:1425-40.

Van Eekert, M.H.A. Transformation of chlorinated compounds by methanogenic granular sludge. PhD Thesis, Wageningen University, Wageningen. 1999; pp. 149.

Von Canstein H, Ogawa J, Shimizu S, Lloyd JR. Secretion of flavins by Shewanella species and their role in extracellular electron transfer. Appl Environ Microbiol 2008;74:615-23.

Walker R, Gingell R, Murrells DF. Mechanisms of azo reduction by Streptococcus faecalis I. Optimization of assay conditions. Xenobiotica 1971;1:221-9.

Weber EJ. Iron-mediated reductive transformation: investigation of reaction mechanism. Environ Sci Technol 1996;30:716-9.

Workman DJ, Woods SL, Gorby YA, Fredrickson JK, Truex MJ. Microbial reduction of vitamin B12 by Shewanella alga strain BrY with subsequent transformation of carbon tetrachloride. Environ Sci Technol 1997;31:2292-7.

Yemashova N, Kalyuzhnyi S. Microbial conversion of selected azo dyes and their breakdown products. Water Sci Technol 2006;53:163-71.

Yu J, Wang X, Yue PL. Optimal decolorization and kinetic modeling of synthetic dyes by Pseudomonas strains. Water Res 2001;35:3579-86.

Yudov MV, Zhilin DM, Pankova AP, Rusanov AG, Perminova IV, Petrosyan VS, et al. Synthesis, metal-binding properties and detoxifying ability of sulphonated humic acids. In: Perminova IV, Hatfield K, Hertkorn N, editors. Use of humic substances to remediate polluted environments: from theory to practice, vol. 52. Dordrecht, The Netherlands: Springer; 2005. p. 485-98.

Zhang YQ Zahir ZA, Amrhein C, Chang A, Frankenberger WT. Application of redox mediator to accelerate selenate reduction to elemental selenium by Enterobacter taylorae. J Agric Food Chem 2007;55:5714-7.

Zou S, Stensel HD, Ferguson JF. Carbon tetrachloride degradation: effect of microbial growth substrate and vitamin B12 content. Environ Sci Technol 2000;34:1751-7. 